\title{
LA-UR-14-29190
}

Approved for public release; distribution is unlimited.

Title: $\quad$ Active and passive meta-surfaces and their interaction with terahertz waves

Author(s): $\quad$ Azad, MD Abul Kalam

Intended for: $\quad$ Seminar at physics department, University of Dhaka

Issued: 
Disclaimer:

Los Alamos National Laboratory, an affirmative action/equal opportunity employer,is operated by the Los Alamos National Security, LLC for the National NuclearSecurity Administration of the U.S. Department of Energy under contract DE-AC52-06NA25396. By approving this article, the publisher recognizes that the U.S. Government retains nonexclusive, royalty-free license to publish or reproduce the published form of this contribution, or to allow others to do so, for U.S. Government purposes. Los Alamos National Laboratory requests that the publisher identify this article as work performed under the auspices of the U.S. Departmentof Energy. Los Alamos National Laboratory strongly supports academic freedom and a researcher's right to publish; as an institution, however, the Laboratory does not endorse the viewpoint of a publication or guarantee its technical correctness. 


\section{Active and passive meta-surfaces and their interaction with terahertz waves}

\section{$\underline{\text { Abul K. Azad }}$}

Center for Integrated Nanotechnologies

Los Alamos National Laboratory

aazad@lanl.gov 


\section{Center for Integrated Nanotechnologies}

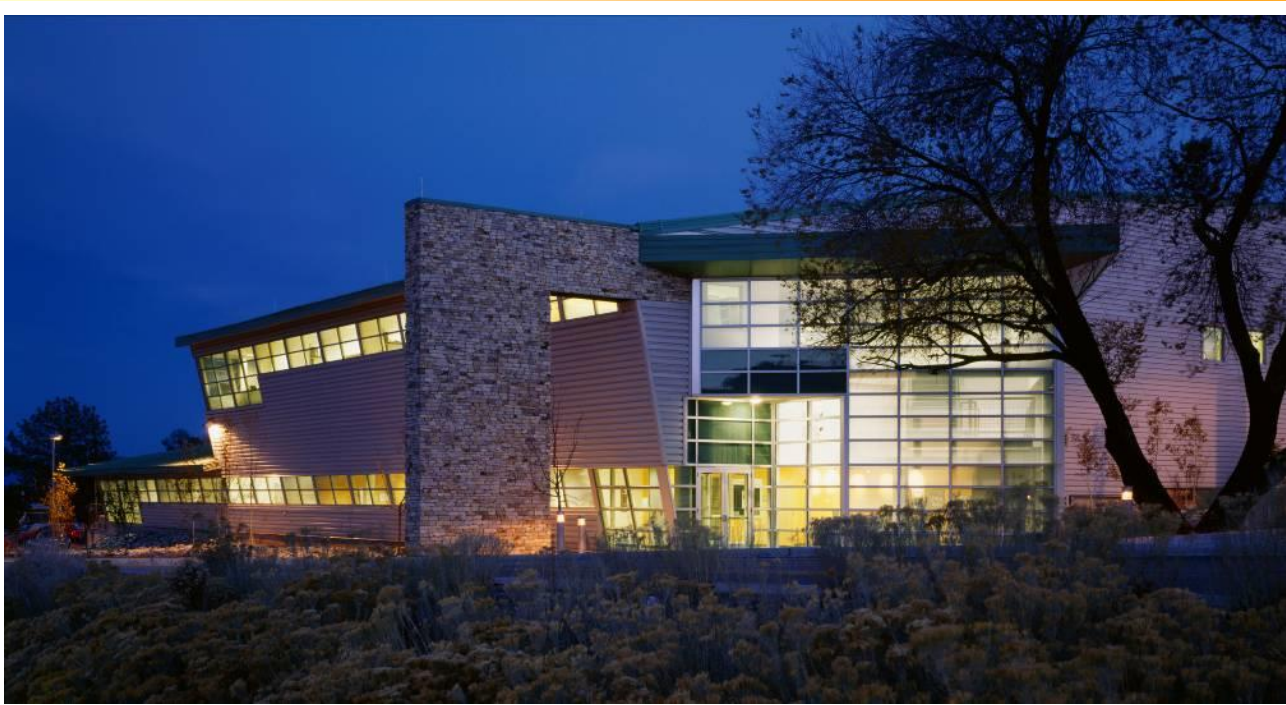

"One scientific community focused on nanoscience integration"

Los Alamos National Laboratory

State-of-the-art facilities

$>$ Leverages LANL and Sandia capabilities

$>$ Develop innovative approaches to nanoscale integration

http://CINT.lanl.gov

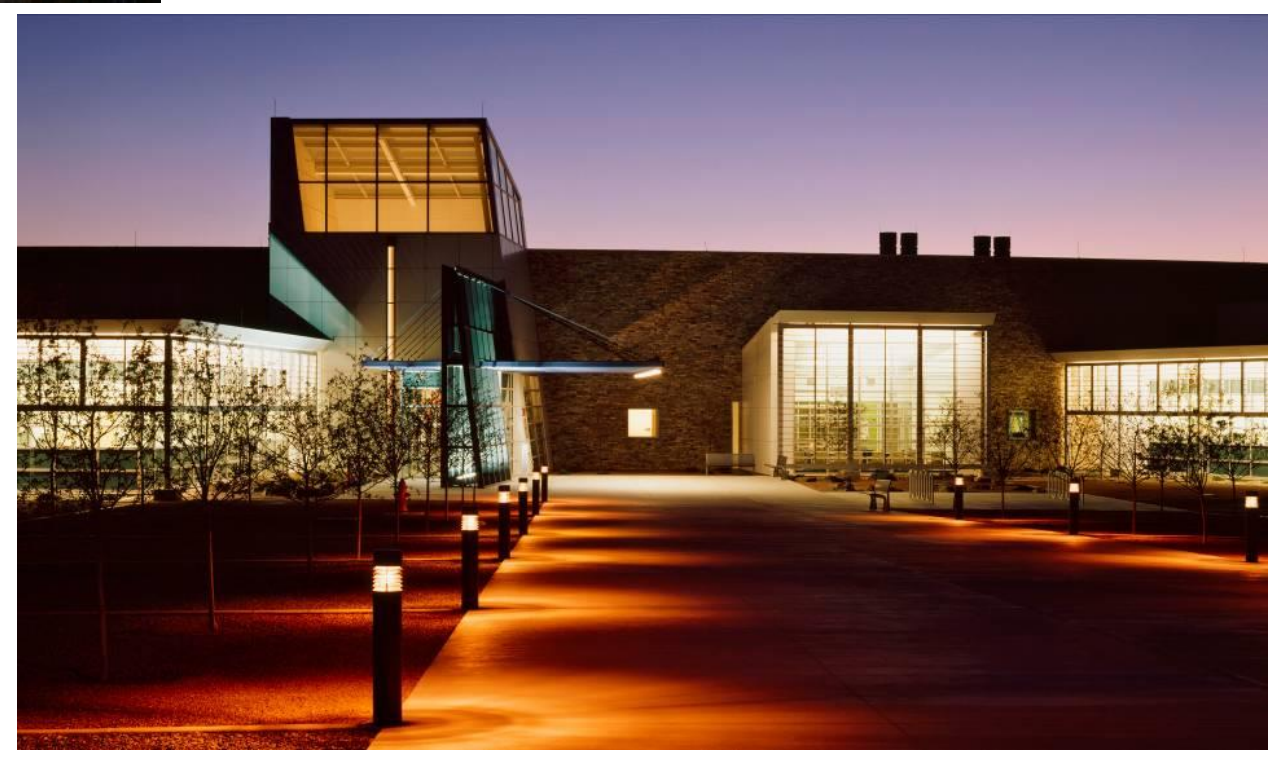

Sandia National Laboratory 


\section{Outline}

$>$ Introduction of metamaterials

$>$ Why terahertz frequency?

$>$ Passive THz metamaterial

$\square$ First realization of terahertz metamaterials

$\square$ Understanding the inter-elemental coupling

$>$ Active terahertz metamaterial

$\square$ Optically tunable modulator

$\square$ Electrically reconfigurable metamaterial

> Ultrathin Terahertz polarization converter

$>$ Future direction 


\section{Metamaterials}

\section{Metamaterials:}

$>$ Metamaterials represents the artificial or engineered materials that have properties beyond the natural materials

\begin{tabular}{|l|l|}
\hline \multicolumn{1}{|c|}{ Natural Materials } & \multicolumn{1}{|c|}{ Metamaterials } \\
\hline $\begin{array}{l}\text { Electromagnetic interaction depends } \\
\text { on chemical composition of the } \\
\text { materials }\end{array}$ & $>\begin{array}{l}\text { Electromagnetic interaction } \\
\text { depends on artificial resonators } \\
\text { Provide unique capability to design } \\
\text { material properties } \\
\text { Scalable through wide range of EM } \\
\text { spectrum }\end{array}$ \\
\hline
\end{tabular}




\section{Interaction of light with natural material}

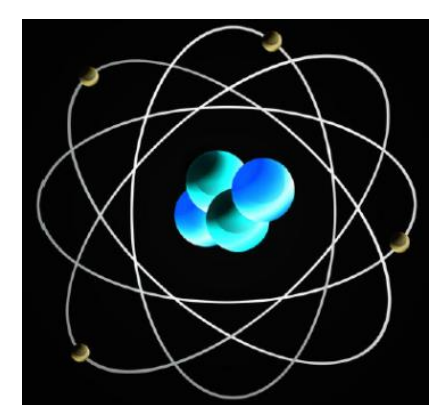

$>$ Electromagnetic interaction depends on chemical composition of the materials

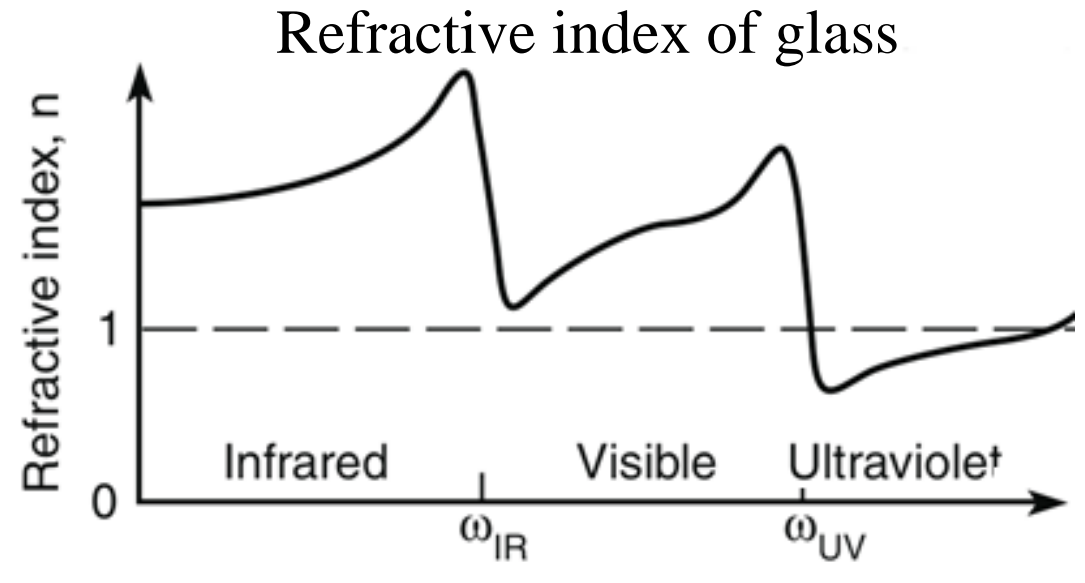

$>$ Resonance at UV - electronic transition

$>$ Resonance at IR $-\mathrm{Si}$-O-Si bonds

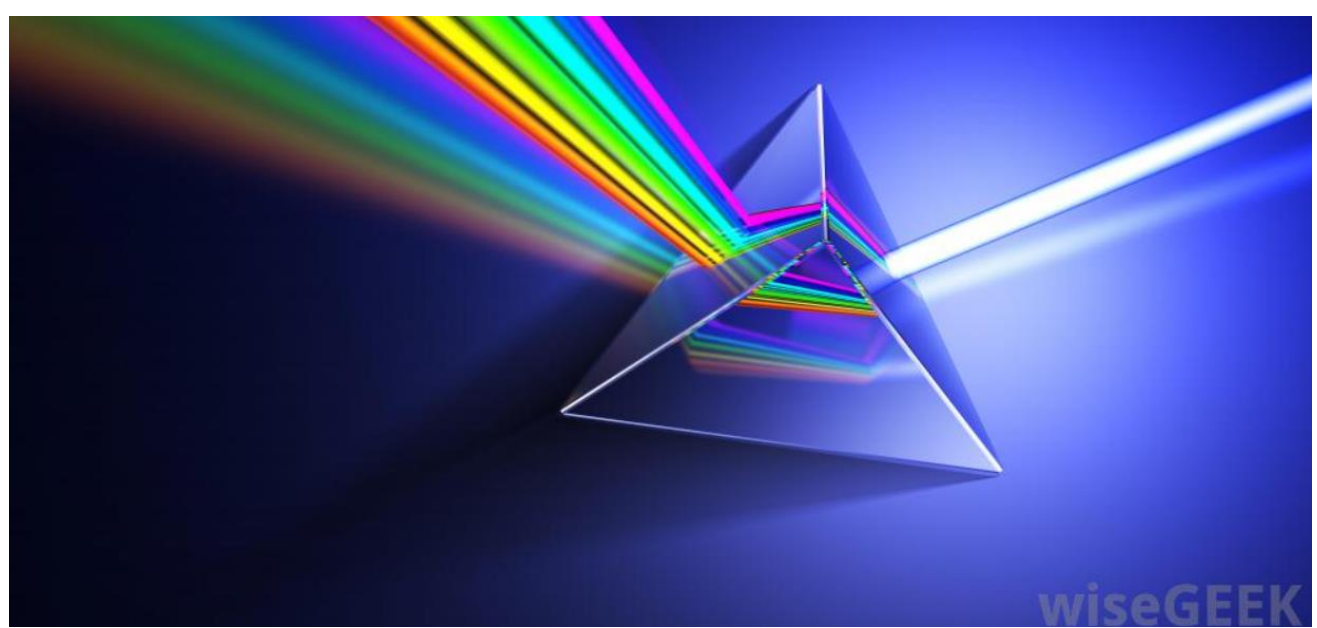




\section{Refraction of light}
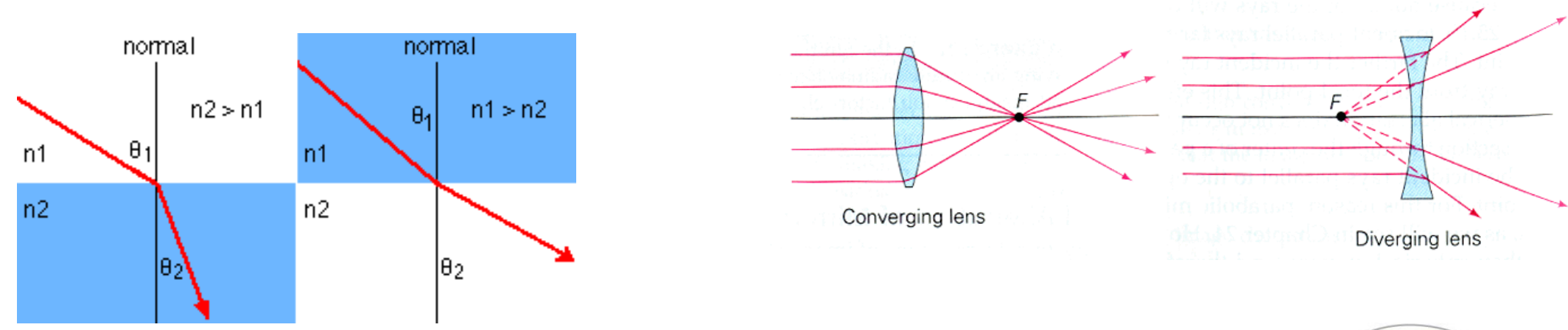

Snell's Law:

$n_{1} \operatorname{Sin} \theta_{1}=n_{2} \operatorname{Sin} \theta_{2}$
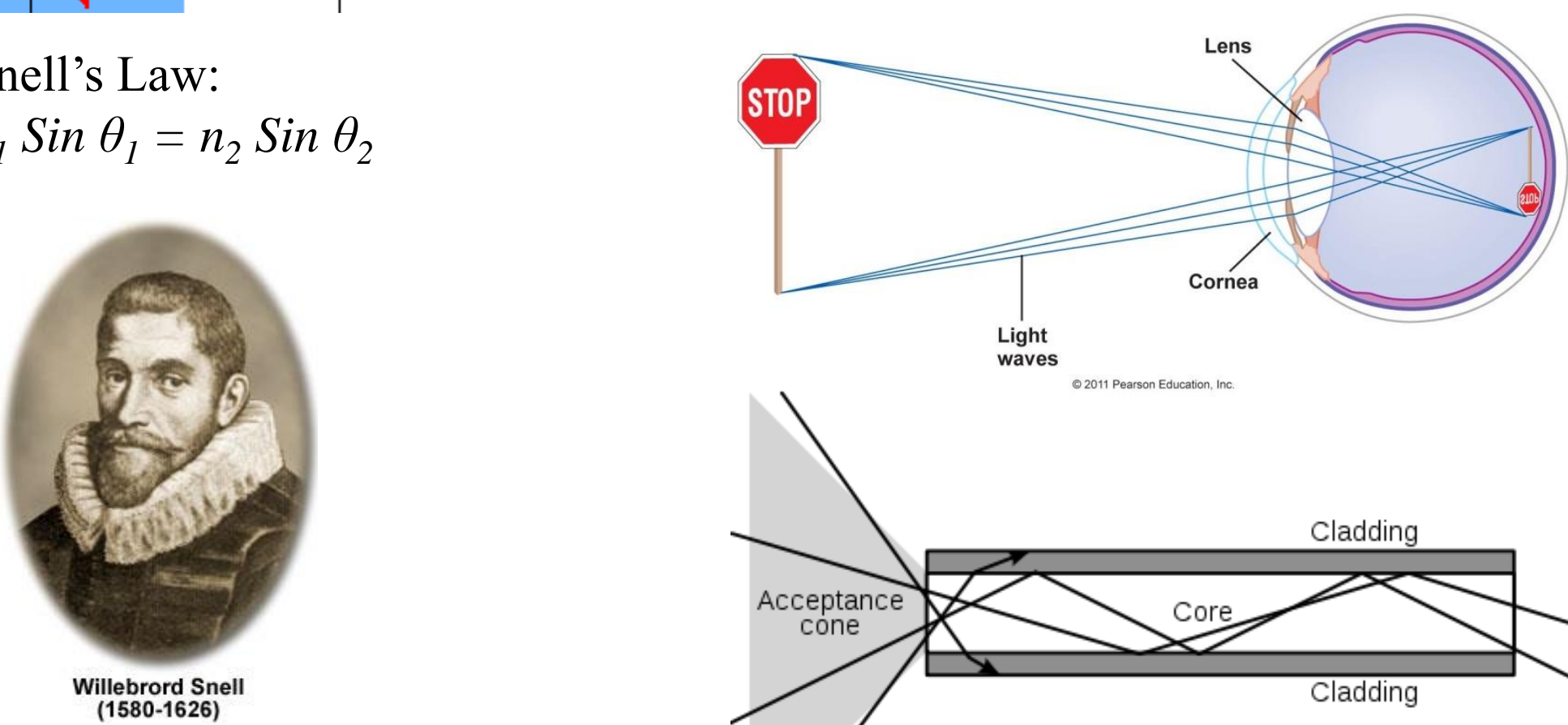

(1580-1626)

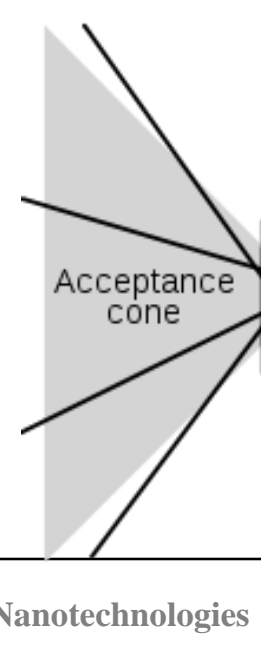

Cladding 


\section{Negative Index Materials}

SOVIET PHYSICS USPEKHI

538.30
VOLUME 10, NUMBER 4

JANUARY-FEBRUARY 1968

THE ELECTRODYNAMICS OF SUBSTANCES WITH SIMULTANEOUSLY NEGATIVE

$$
V A L U E S O F \in A N D \mu
$$

V. G. VESELAGO

P. N. Lebedev Physics Institute, Academy of Sciences, U.S.S.R.

Usp. Fiz. Nauk 92, 517-526 (July, 1964)

$>$ Light bends to the "wrong" direction, i.e. negative index of refraction

$>$ Left-handed materials with opposite phase and group velocities

$>$ Focusing with a flat slab, convex and concave lenses change places

No natural material has been found to exhibit such properties !!
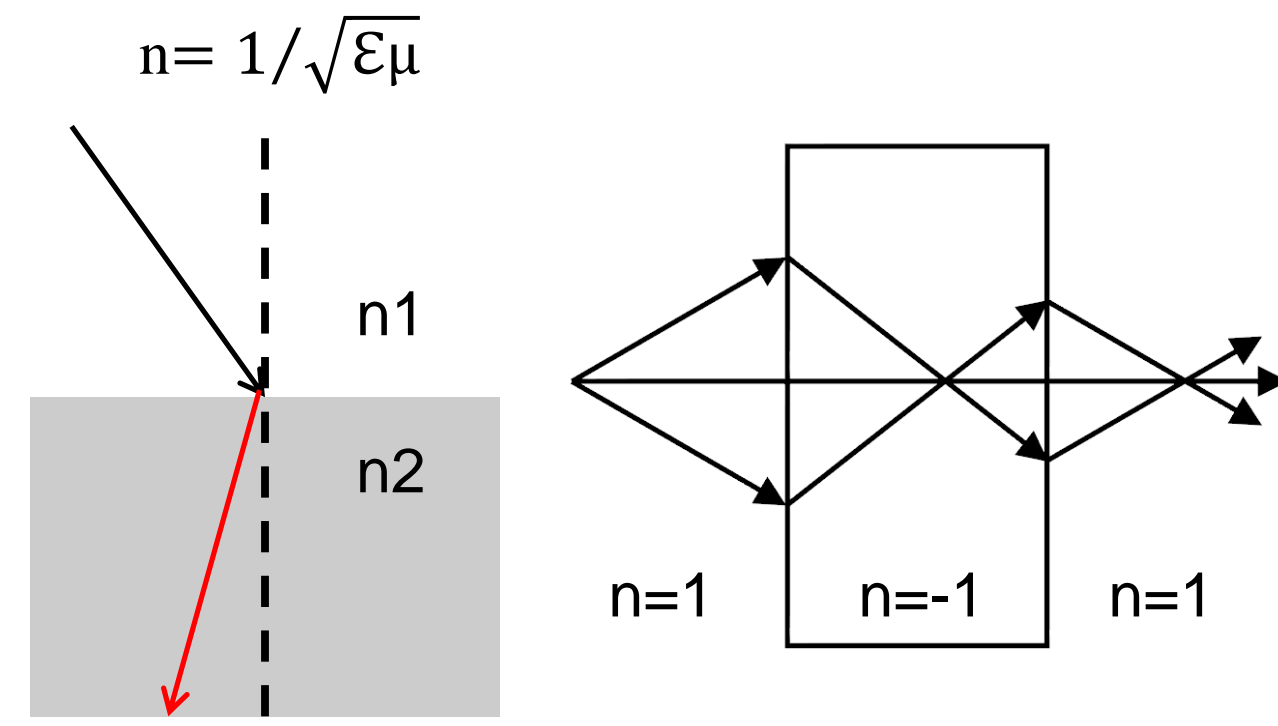


\section{Controlling the Effective Electric and Magnetic Response}
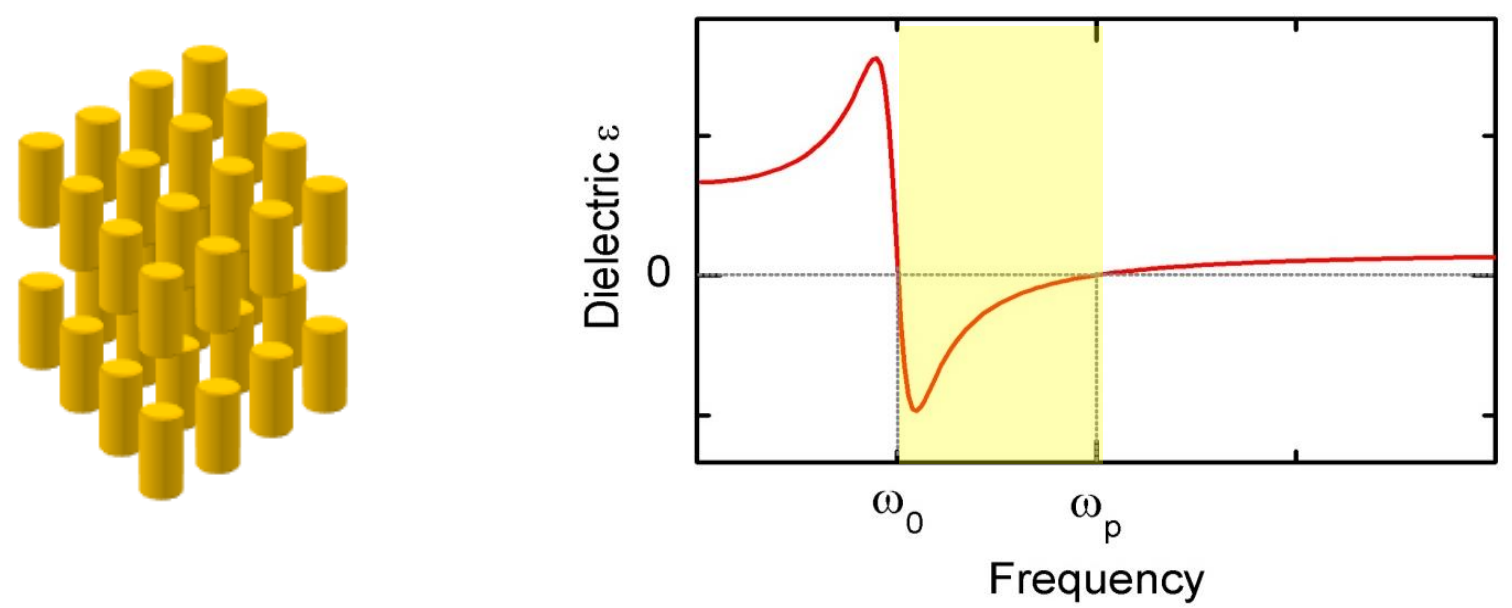

$$
\varepsilon(\omega)=1-\frac{\omega_{p}^{2}-\omega_{0}^{2}}{\omega^{2}-\omega_{0}^{2}+i \omega \Gamma}
$$
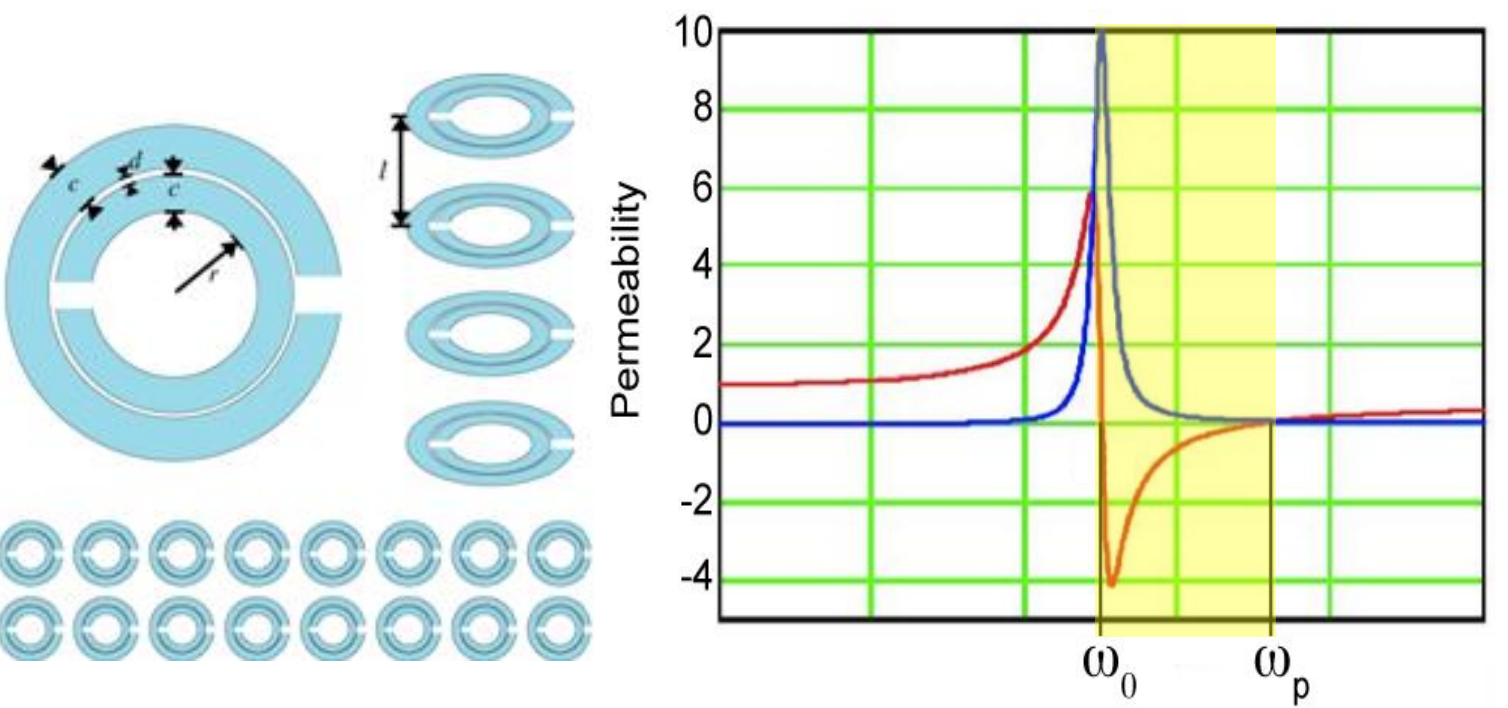

$$
\mu_{e f f}=1-\frac{\frac{\pi r^{2}}{a^{2}}}{1+\frac{2 \sigma i}{\omega r \mu_{0}}-\frac{3}{\pi^{2} \mu_{0} \omega^{2} C r^{3}}}
$$

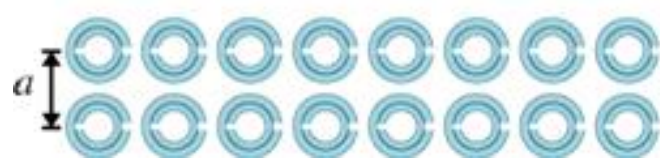

Pendry et al., Phys. Rev. Lett. 76, 4773 (1996), Pendry et al., IEEE Trans. Microwave Tech. 47, 2075 (1999) 


\section{Simultaneously Negative $\varepsilon$ and $\mu$}

\section{Composite Medium with Simultaneously Negative Permeability and Permittivity}

D. R. Smith,* Willie J. Padilla, D.C. Vier, S.C. Nemat-Nasser, and S. Schultz

Department of Physics, University of California, San Diego, 9500 Gilman Drive, La Jolla, California 92093-0319

(Received 2 December 1999)

We demonstrate a composite medium, based on a periodic array of interspaced conducting nonmagnetic split ring resonators and continuous wires, that exhibits a frequency region in the microwave regime with

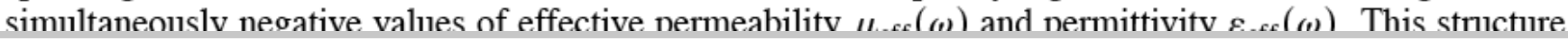

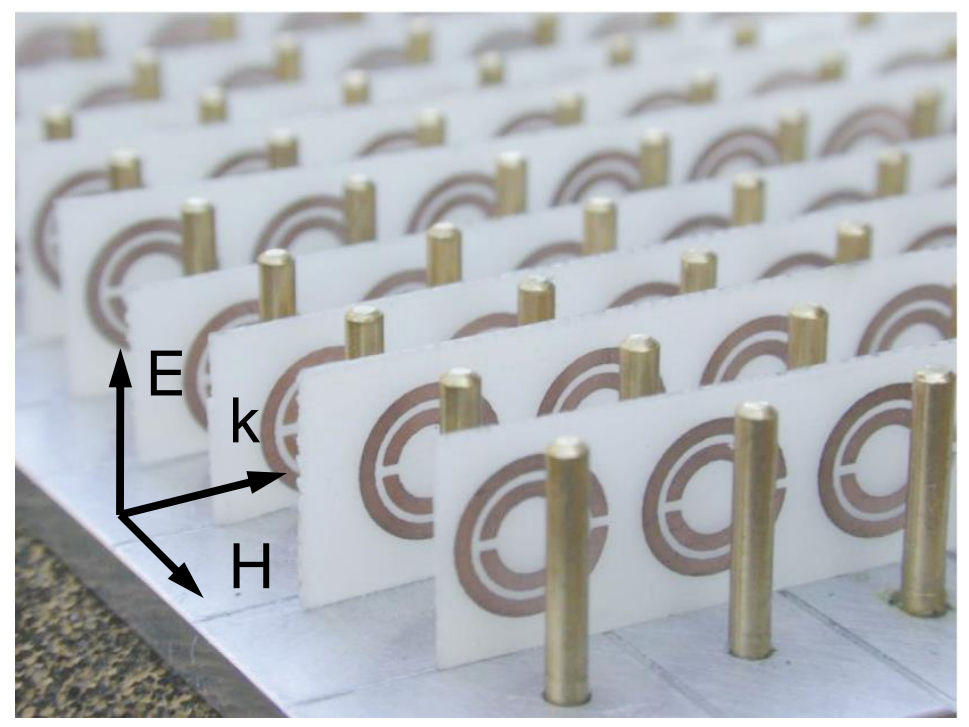

$>$ Negative permittivity $\varepsilon<0$ by metal rods array

$>$ Negative permeability $\mu<0$ by splitring resonators array

$>$ At certain frequency range with simultaneously negative $\varepsilon<0$ and $\mu<$ 0 , microwave can propagate 


\section{Electromagnetic Metamaterials}

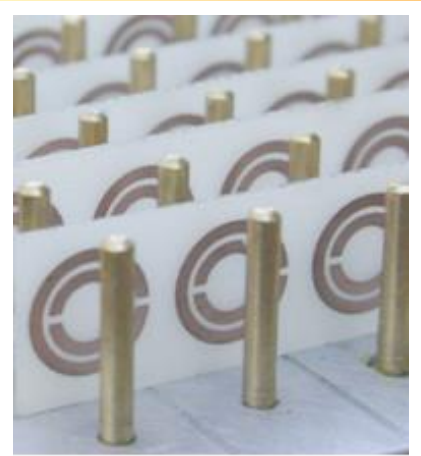

Smith et al., PRL 84 (1999).

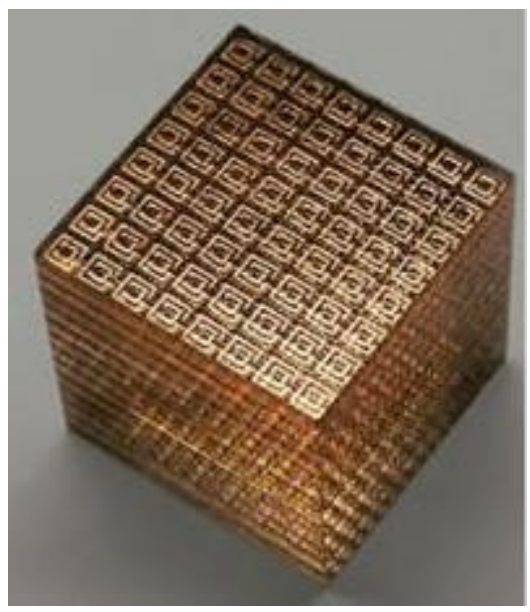

3D metamaterial

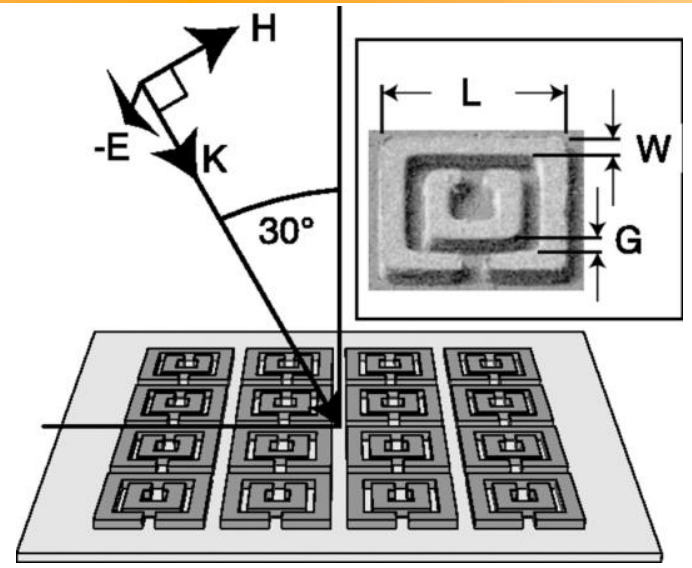

Yen et al., Science 303, 1494 (2004).

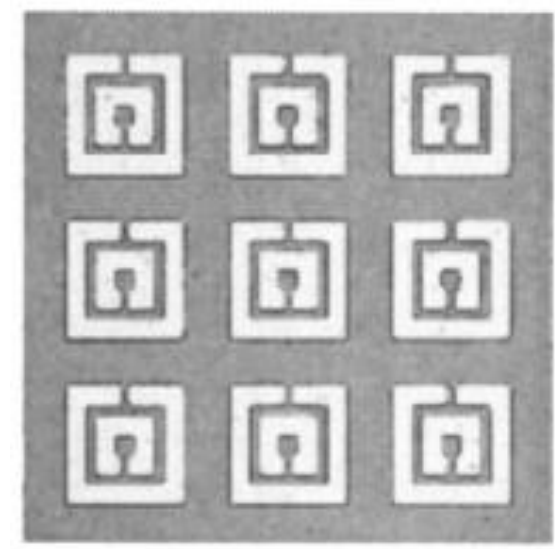

$50 \mu \mathrm{m}$

Azad et al., Opt. Lett. 31, 634 (2006)

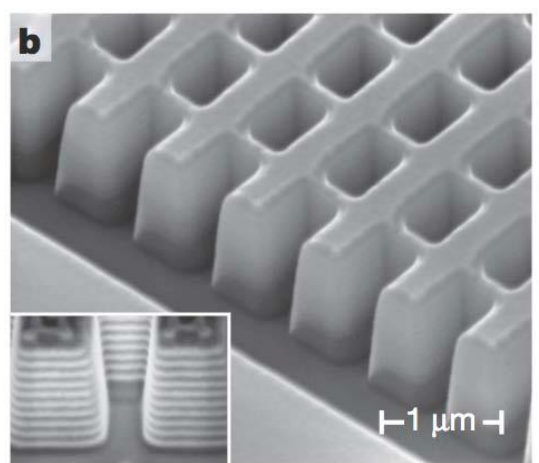

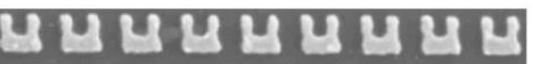
눈느느는 본บบㅂํำ Ч 논 ч분ำ

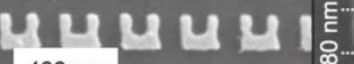
$400 \mathrm{~nm}$ ฯ ル ル $200 \mathrm{~nm}$ 


\section{Negative Refraction}

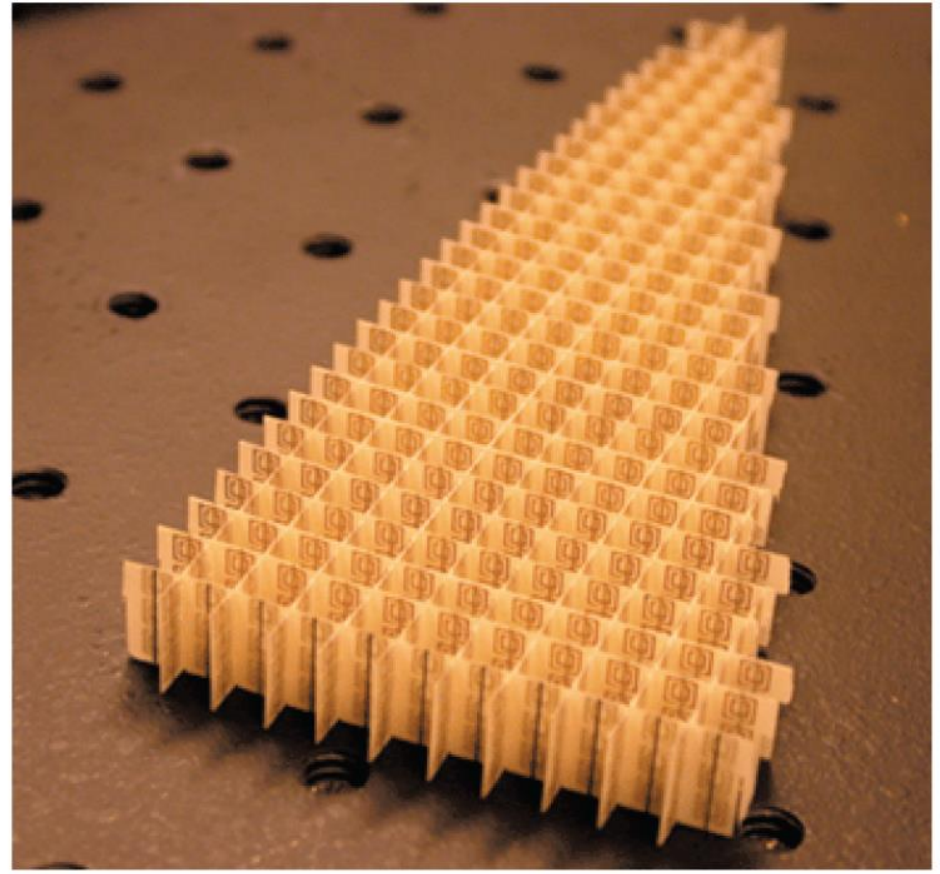

Shelby et al., Science 292, 77 (2001).

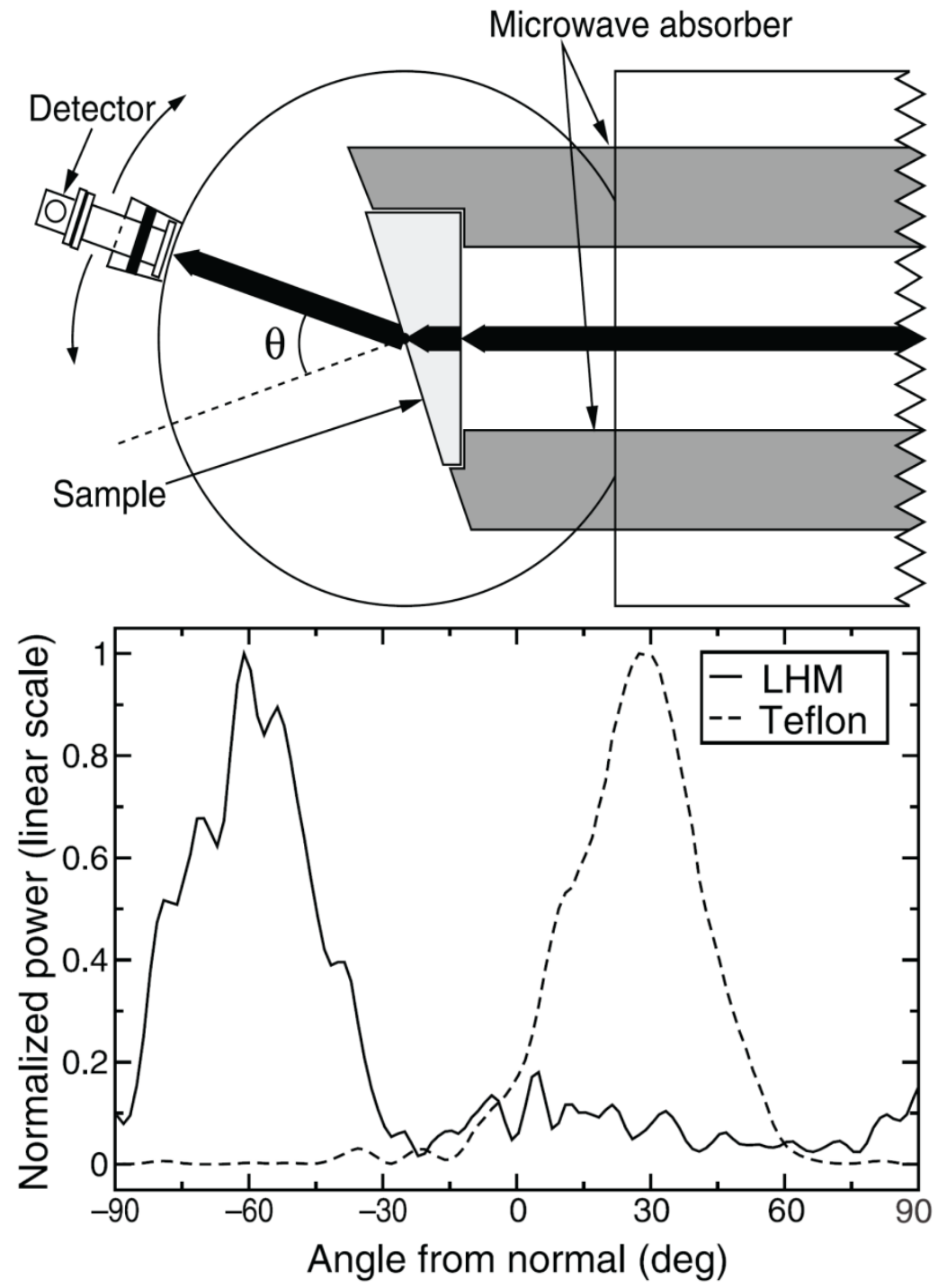




\section{Meta-surface is future for flat integrated optics}

Anomalous light refraction
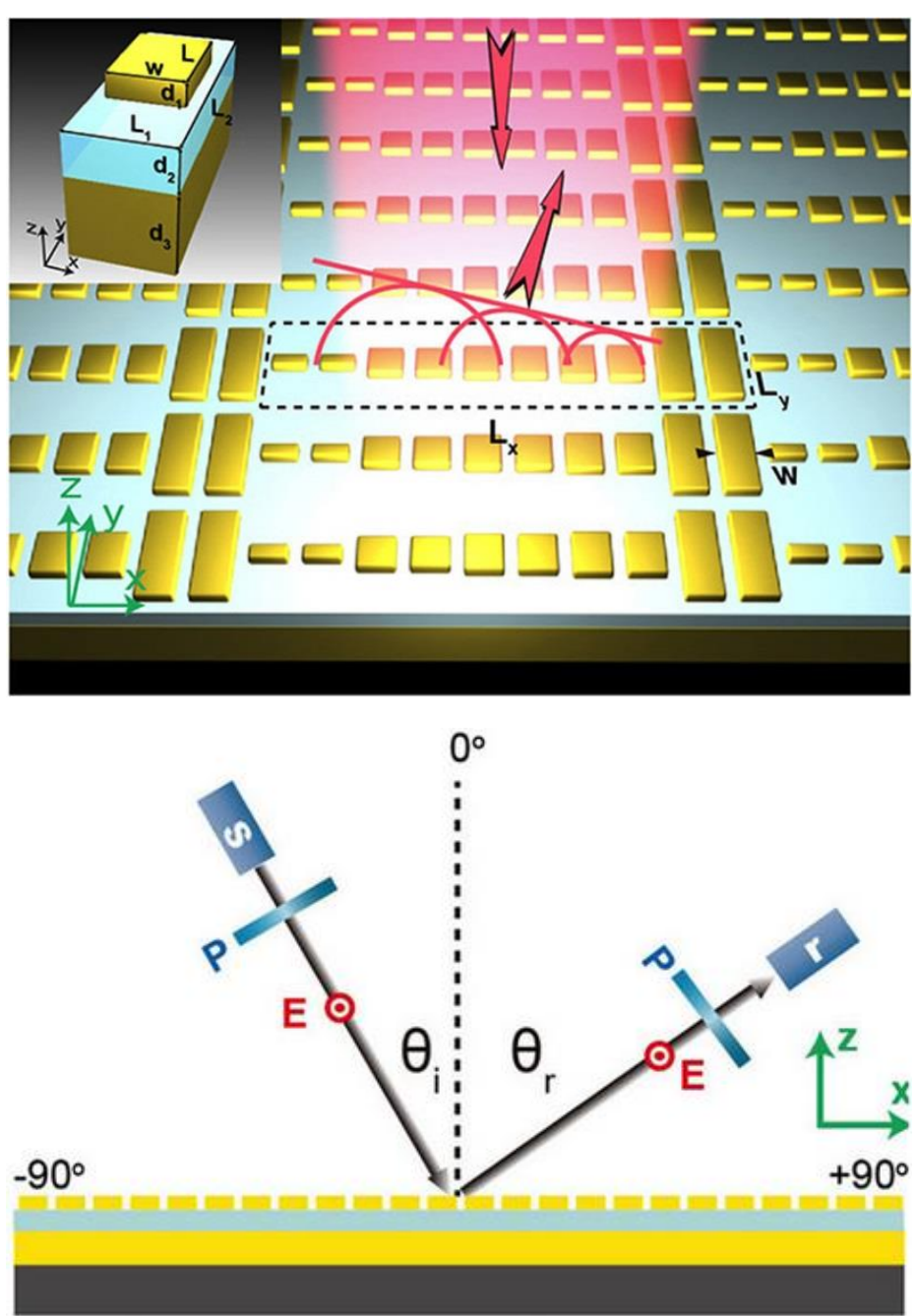

Focusing with a flat lens
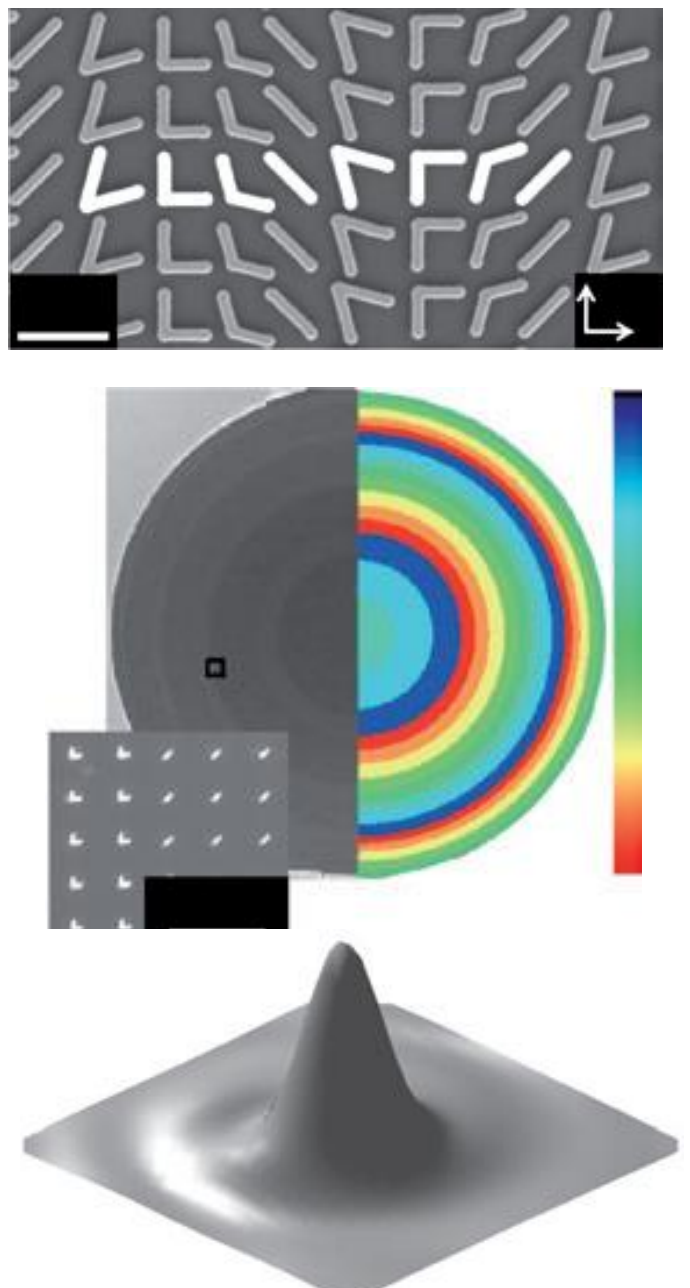

N. Yu, NATURE MATERIALS, 13, 144 (2014) 


\section{Why Terahertz?}

\begin{tabular}{|c|c|c|c|c|c|c|c|}
\hline \multicolumn{6}{|c|}{ Ellestromics } & \multicolumn{2}{|c|}{ Photonics } \\
\hline & \multicolumn{2}{|r|}{ microwave } & \multicolumn{3}{|c|}{ THz Regime } & IR & UV \\
\hline $300 \mathrm{MHz}$ & $3 \mathrm{GHz}$ & $30 \mathrm{GHz}$ & $300 \mathrm{GHz}$ & $3 \mathrm{THz}$ & $30 \mathrm{THz}$ & $300 \mathrm{THz}$ & $3 \mathrm{PHz}$ \\
\hline $1 \mathrm{~m}$ & $10 \mathrm{~cm}$ & $1 \mathrm{~cm}$ & $1 \mathrm{~mm}$ & $100 \mu \mathrm{m}$ & $10 \mu \mathrm{m}$ & $1 \mu \mathrm{m}$ & $100 \mathrm{~nm}$ \\
\hline
\end{tabular}

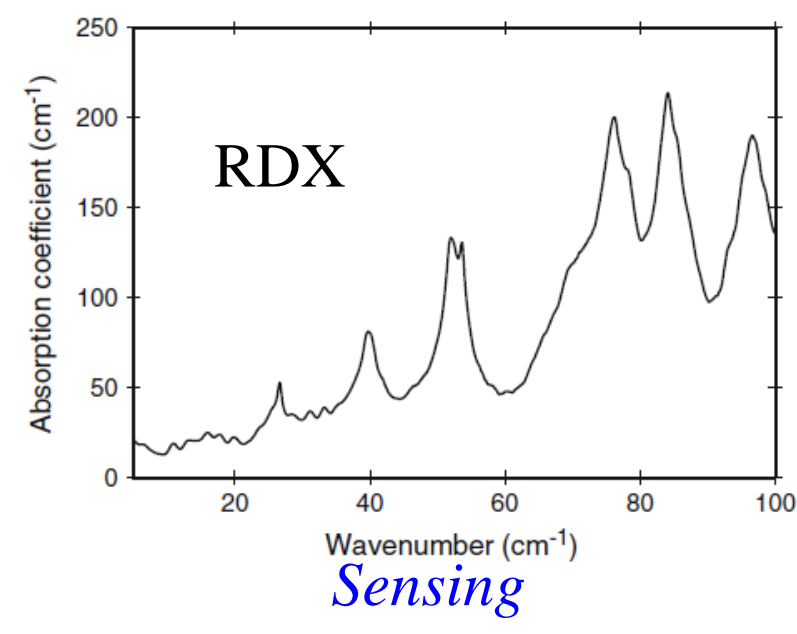

V. Whitley, Anal. Bioanal Chem, 395, 315 (2009)

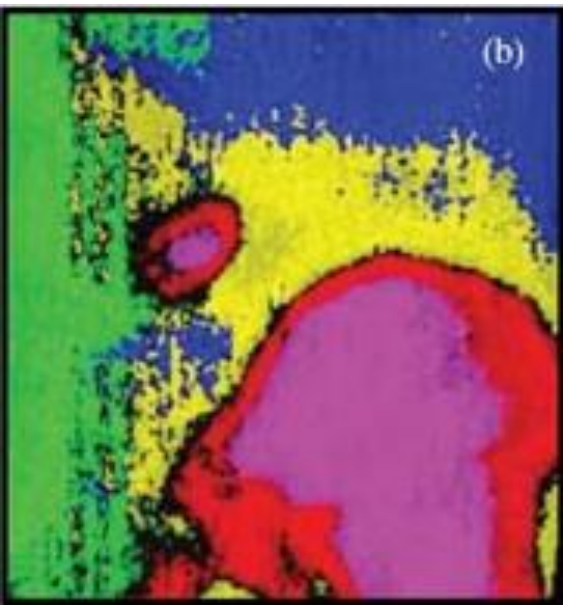

Imaging

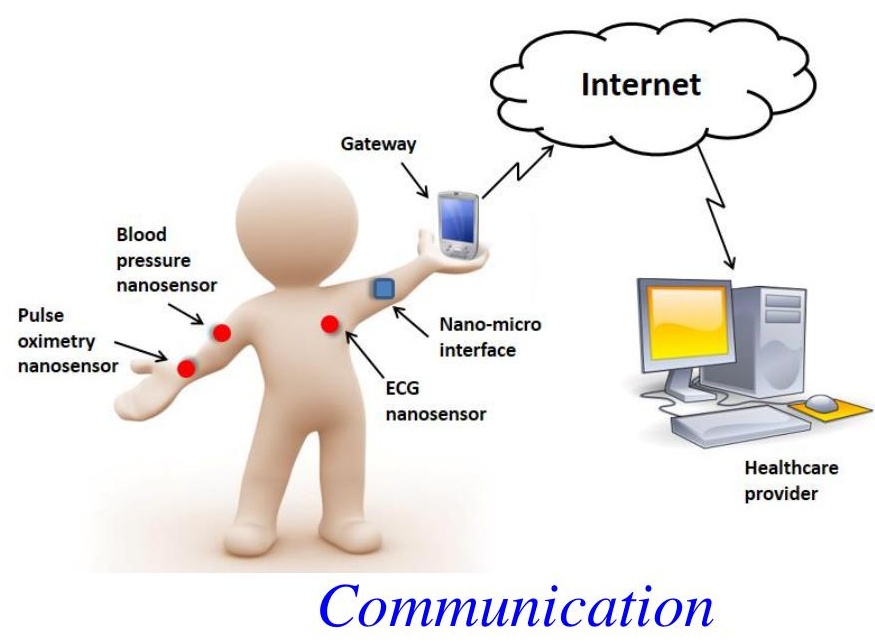

Phys. Med. Biol. 554615 (2010)

$>$ Extremely difficult generation/detection $>$ No practical terahertz devices $>$ THz Gap

Metamaterials are optimistic solutions !! 


\section{Optoelectronic (PC) Terahertz Spectrometer}
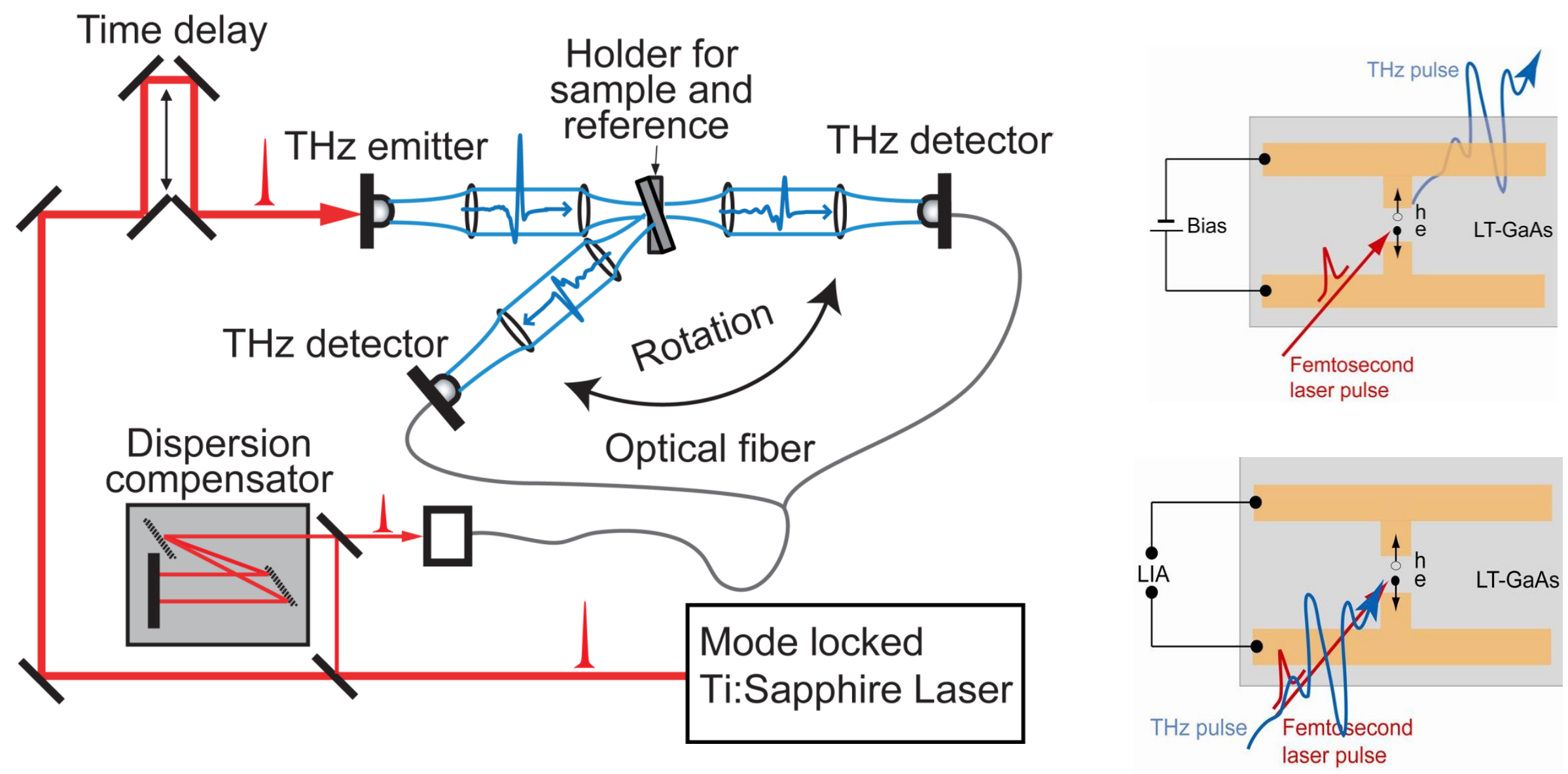


\section{Optical THz Switch: Optical-Pump Terahertz-Probe}

$1 \mathrm{KHz}, \sim 100 \mathrm{fs} \quad$ Beam splitters @ $800 \mathrm{~nm} \longrightarrow$

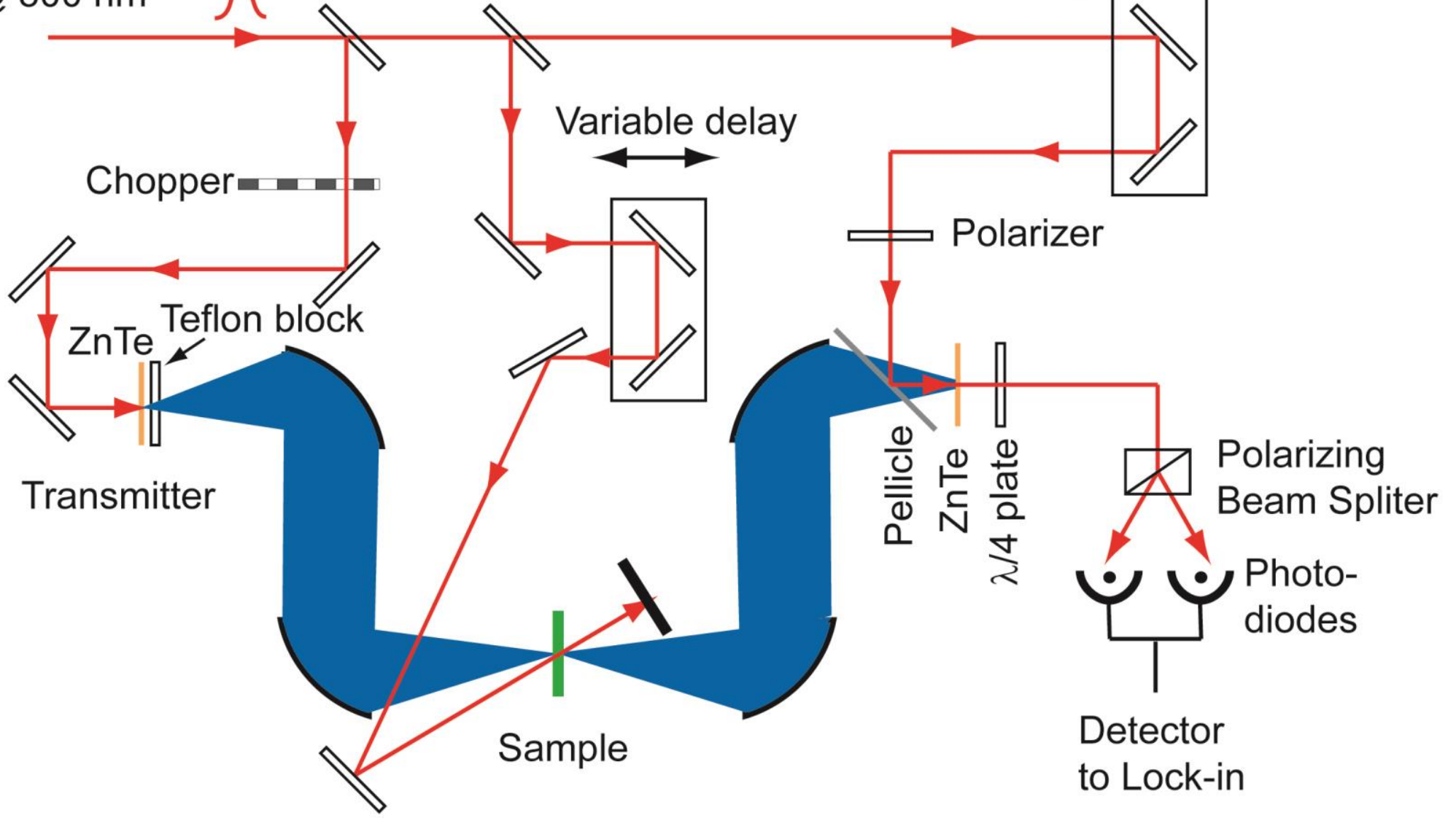




\section{THz pulse to THz spectrum}

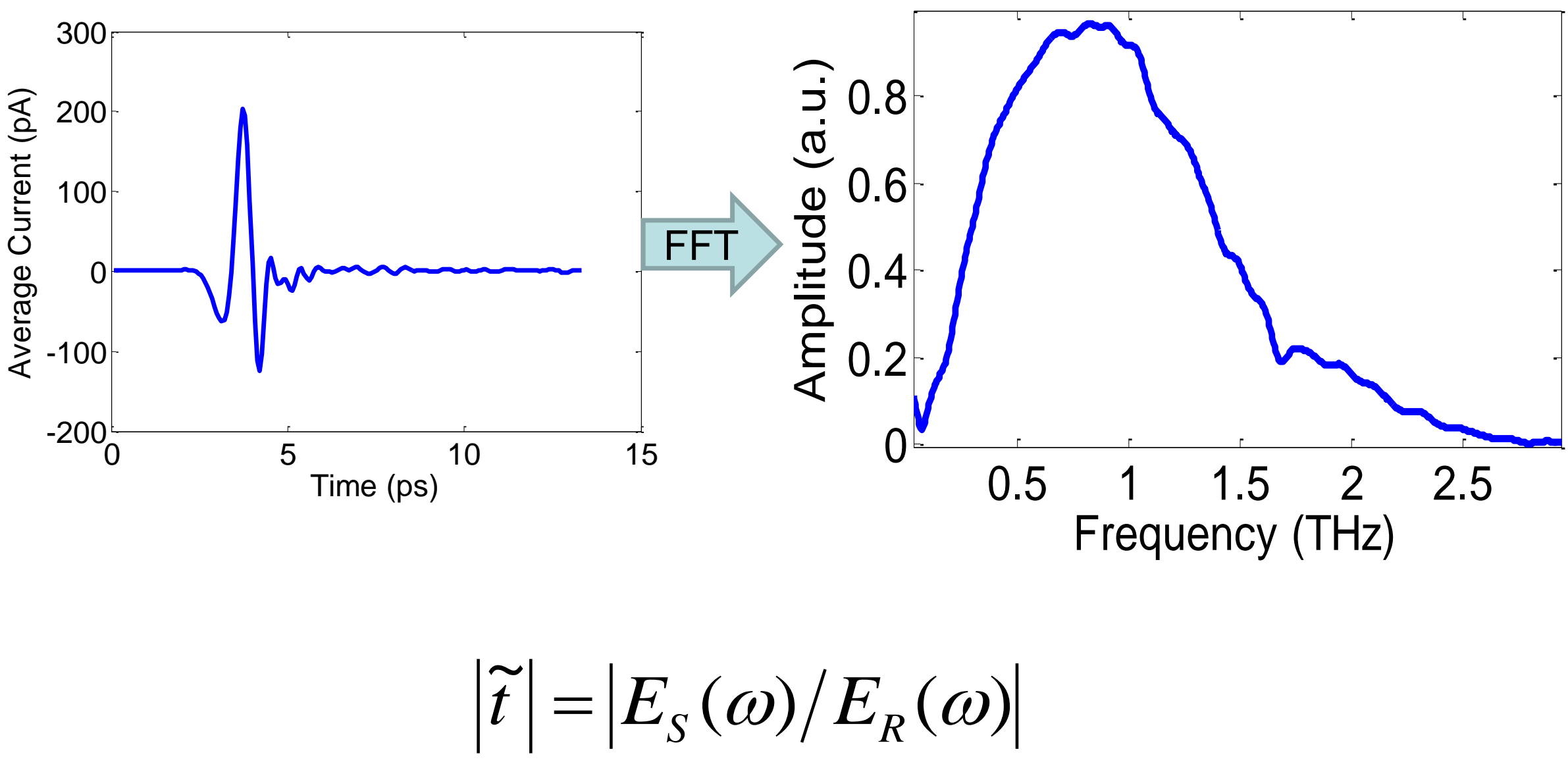




\section{Metamaterial unit cell (SRR)}

$>$ size $\sim \lambda / 10$

$>$ Collectively acts like an effective medium

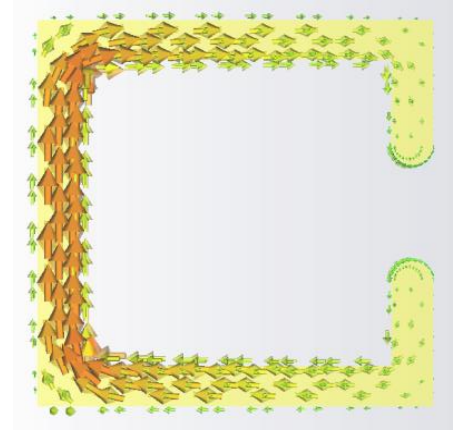

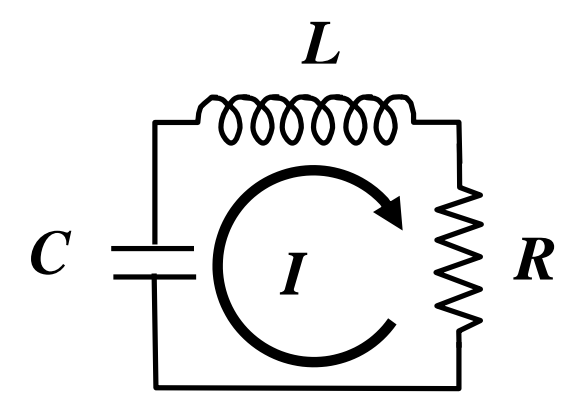

Equivalent circuit

$$
\omega=\frac{1}{\sqrt{L C}}
$$

Resonance frequency

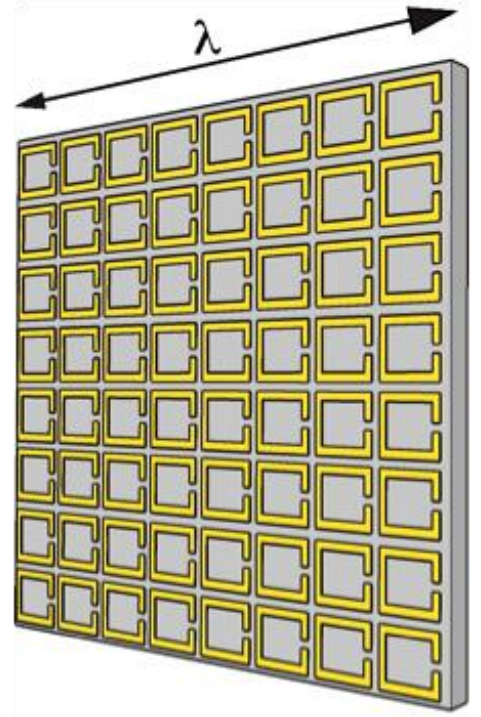

Array of SRR
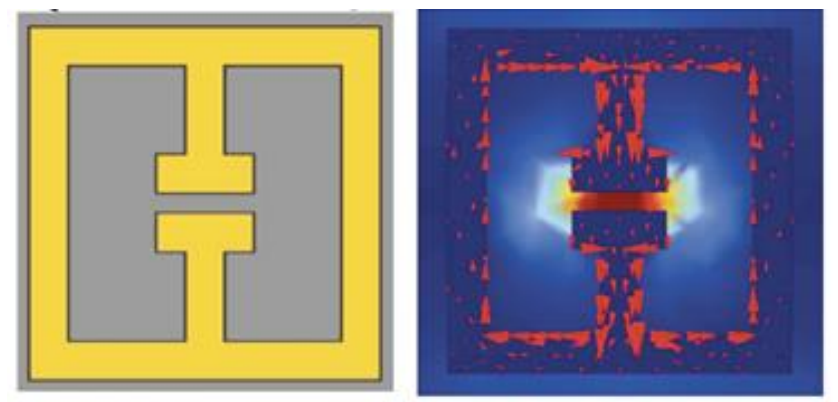

High

Low

$>$ Well disperse effective behavior dominated by individual resonance

$>$ Effective behavior can be altered significantly from individual SRR by inter-elemental interaction 


\section{Realization of terahertz metamaterials}

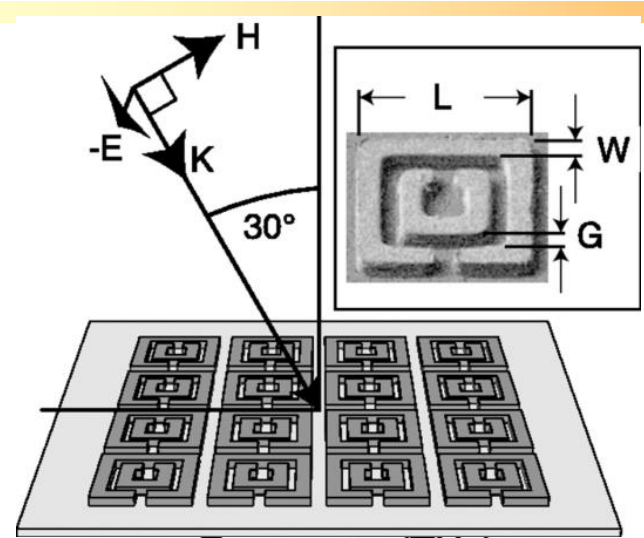

Frequency (THz)

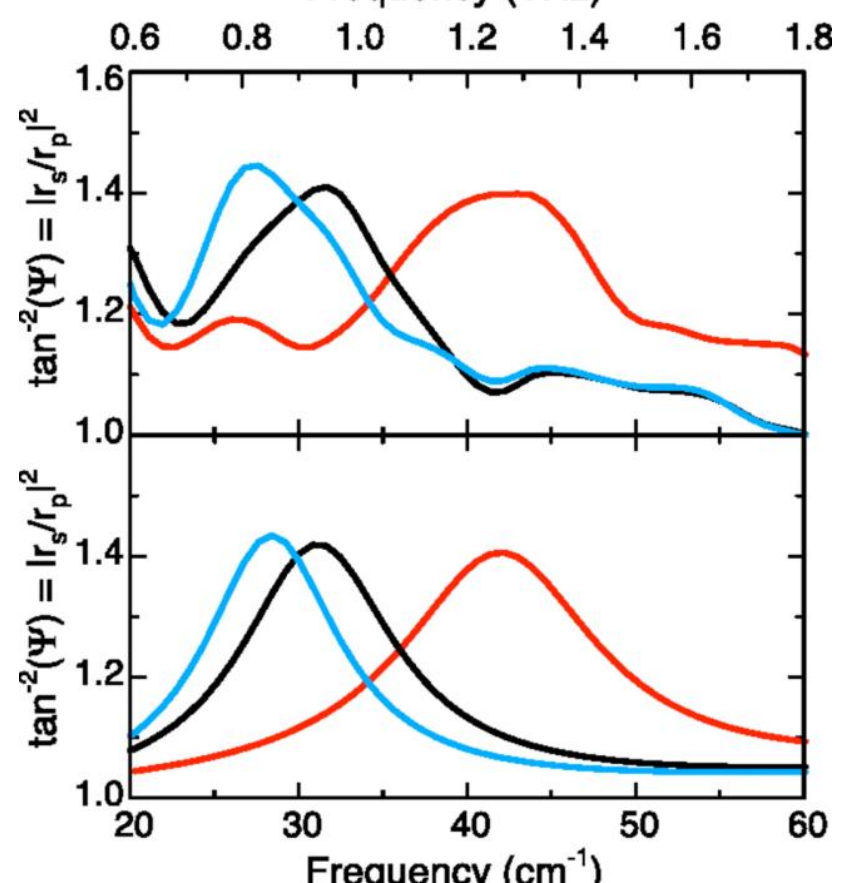

Yen et al., Science 303, 1494 (2004).

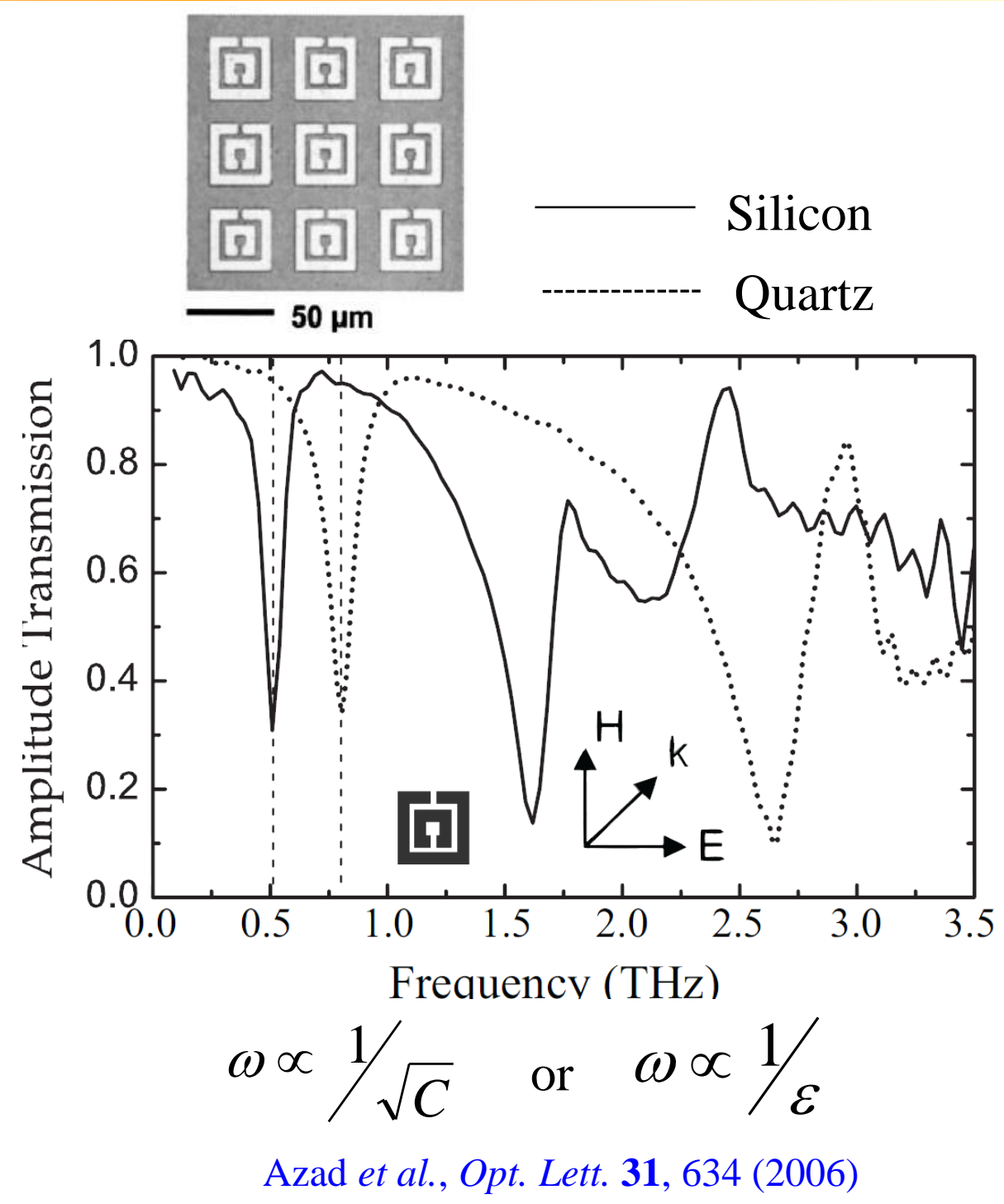




\section{Resonances modes and intermodal interactions}
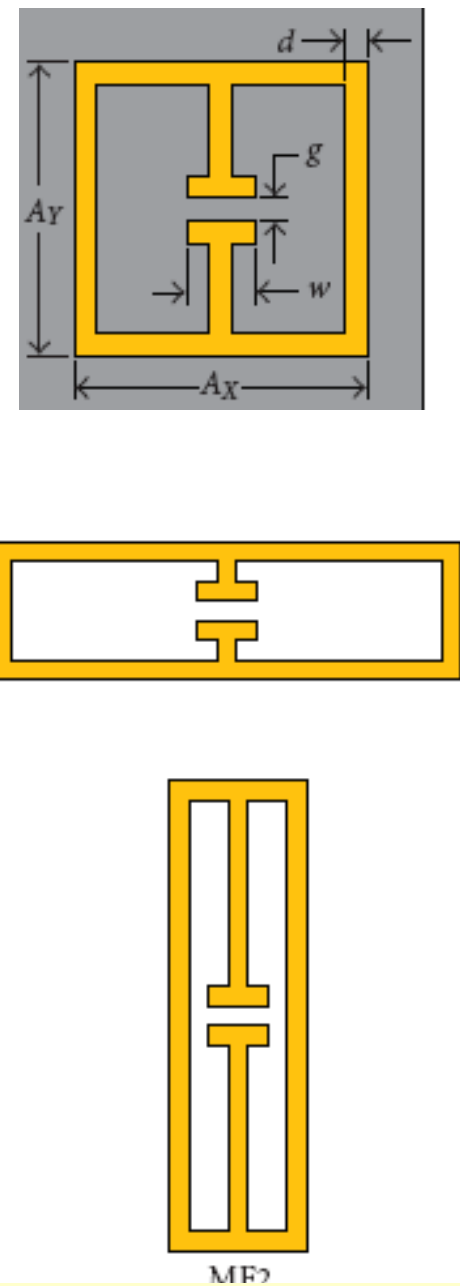

Azad et al., Appl. Phys. Lett. 92, 011119 (2008)

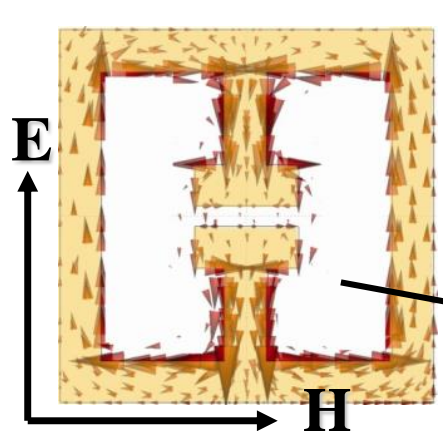

$R L C$ resonance
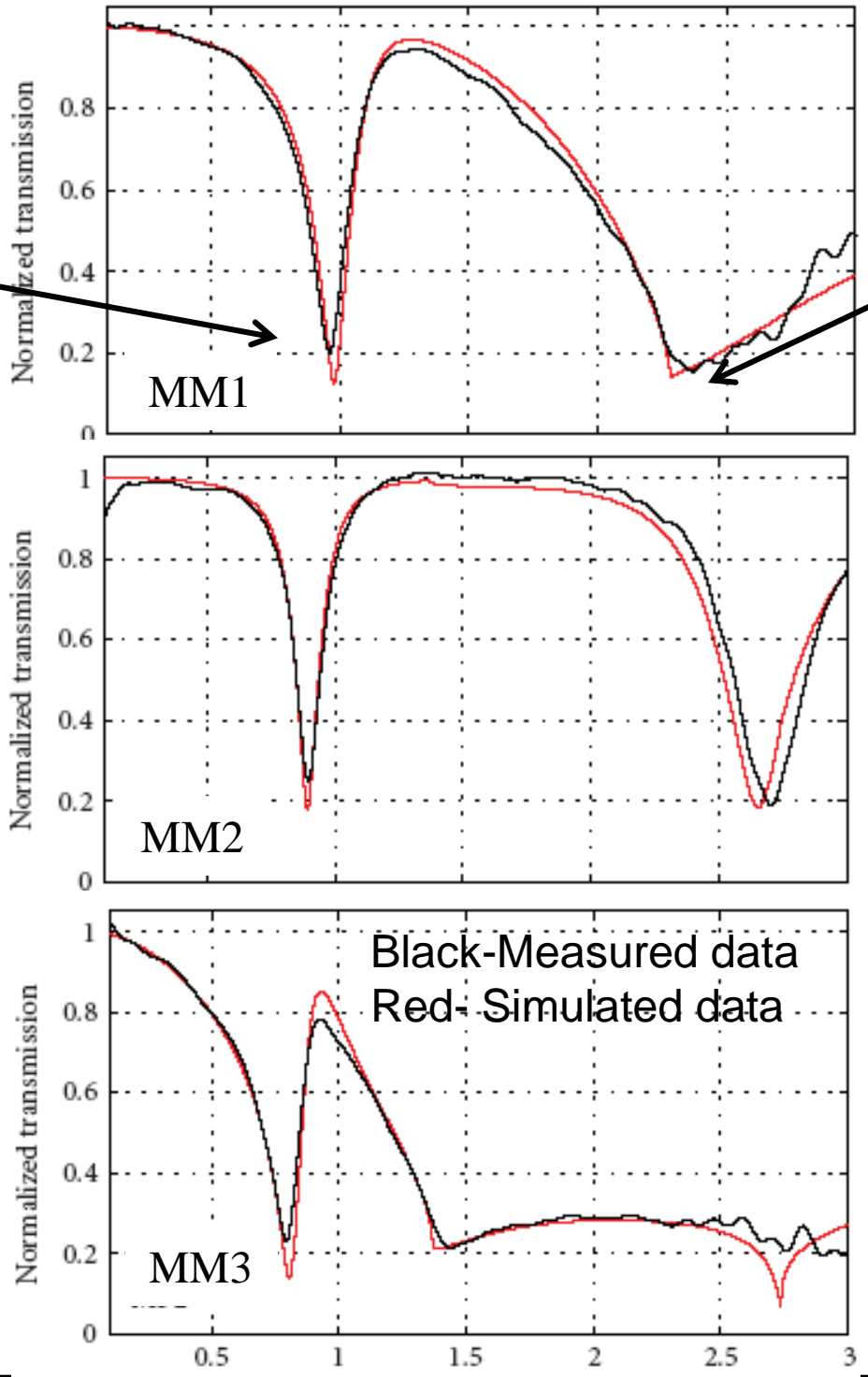

Center for Integrated Nanotechnologies
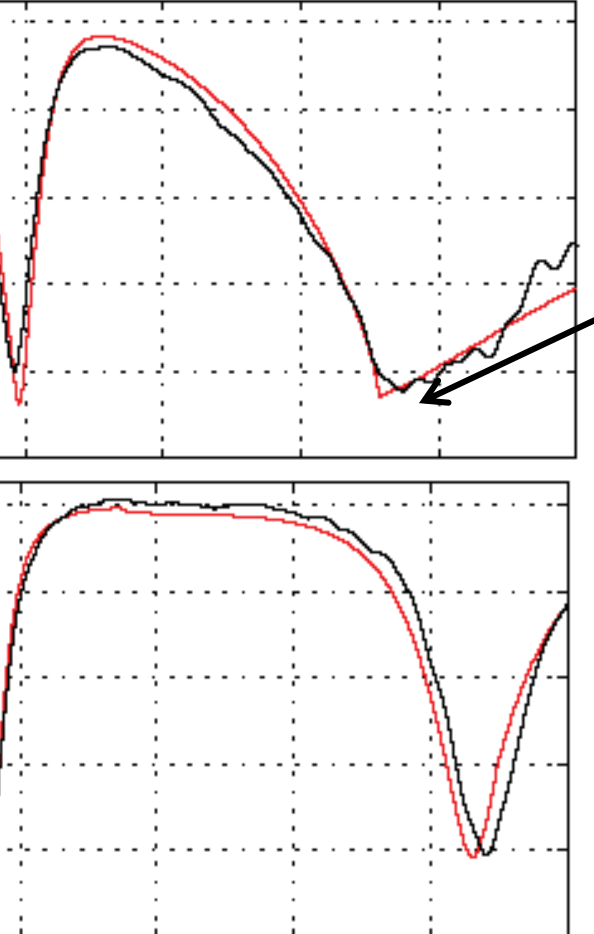

Black-Measured data Red -Simulated data

Collective dipolar resonance 


\section{Coupling between eigen states}

Transmission Line Model

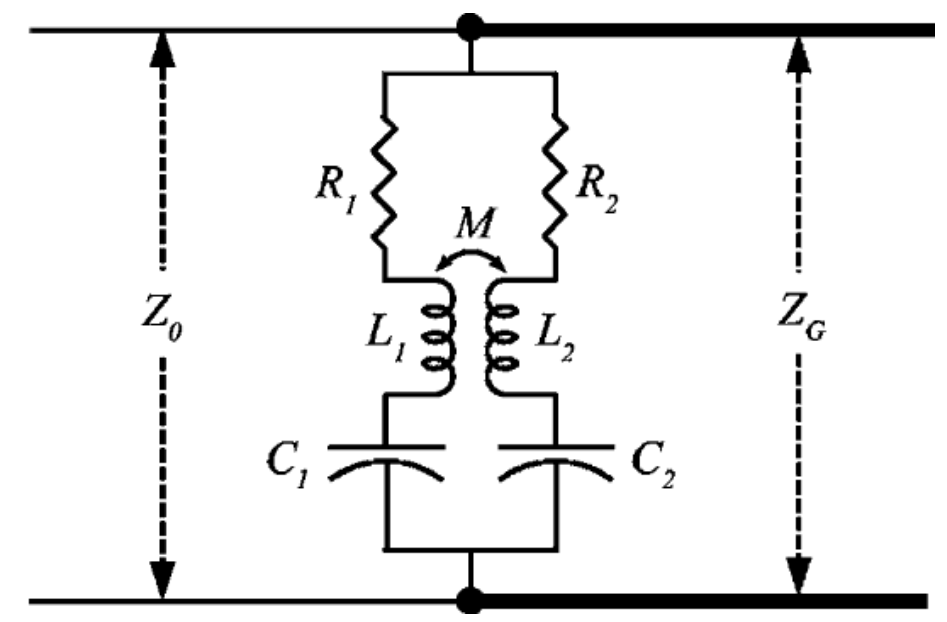

$>\mathrm{LC}$ resonance interacts with dipolar resonance

$>\mathrm{L}$ and $\mathrm{C}$ also varies with shape due to fringing fields

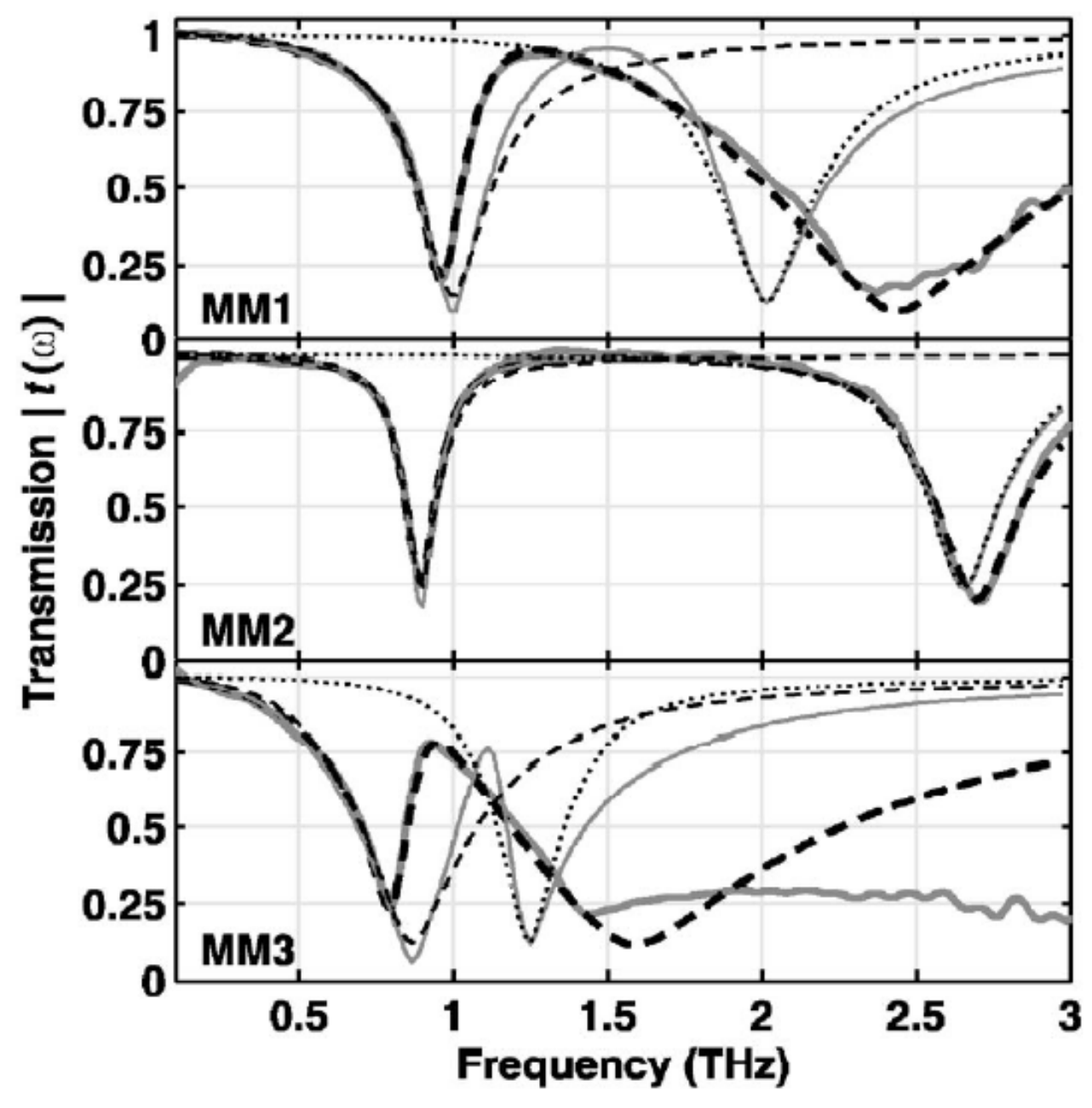

Azad et al., Appl. Phys. Lett. 92, 011119 (2008) 


\section{Effect of Coulomb interaction in coupled MM}
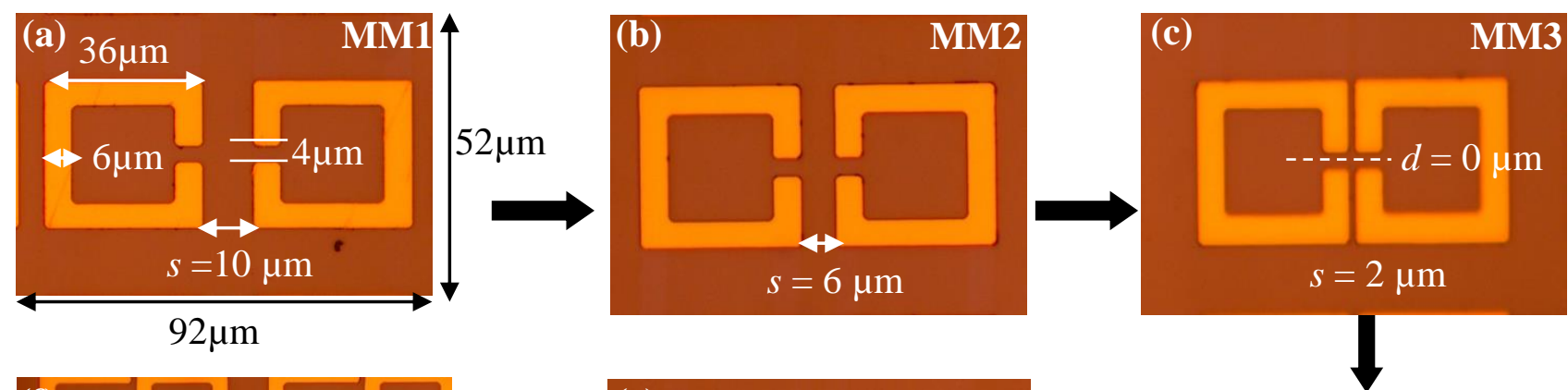

Vary separation $S$
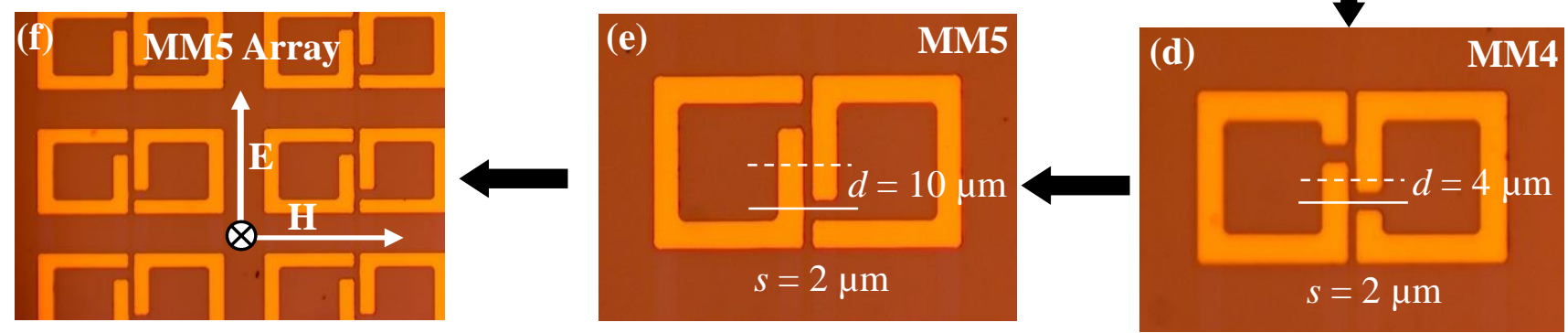

Vary gap asymmetry $d$

D. Roy Chowdhury et al. Opt. Exp. 19, 10679 (2011) 


\section{Effect of Coulomb interaction in coupled MM}
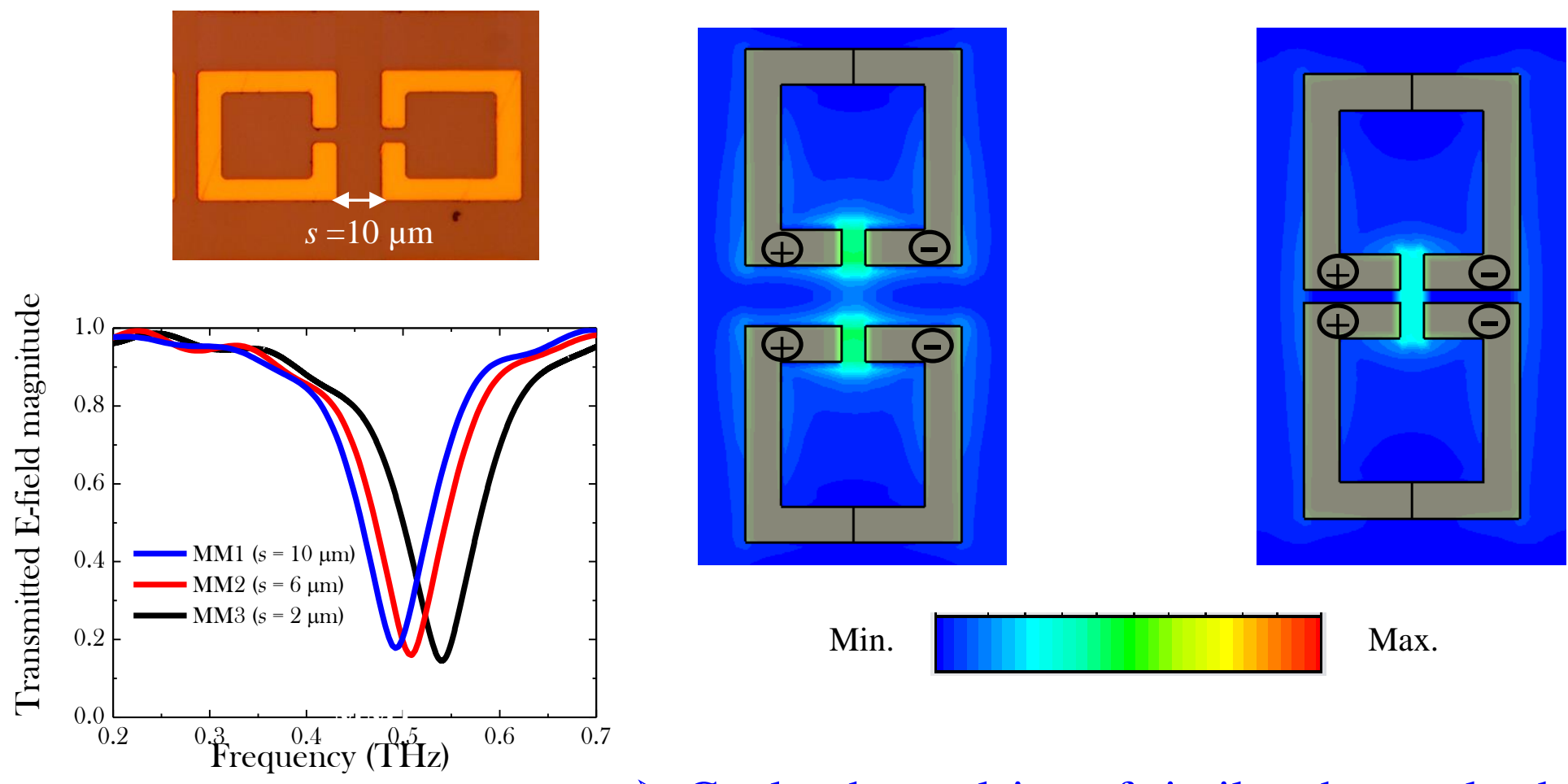

Min.

Max.

Coulomb repulsion of similar charges leads to resonance blue shift

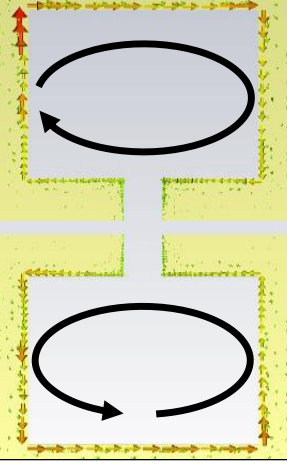




\section{Effect of Coulomb interaction in coupled MM}
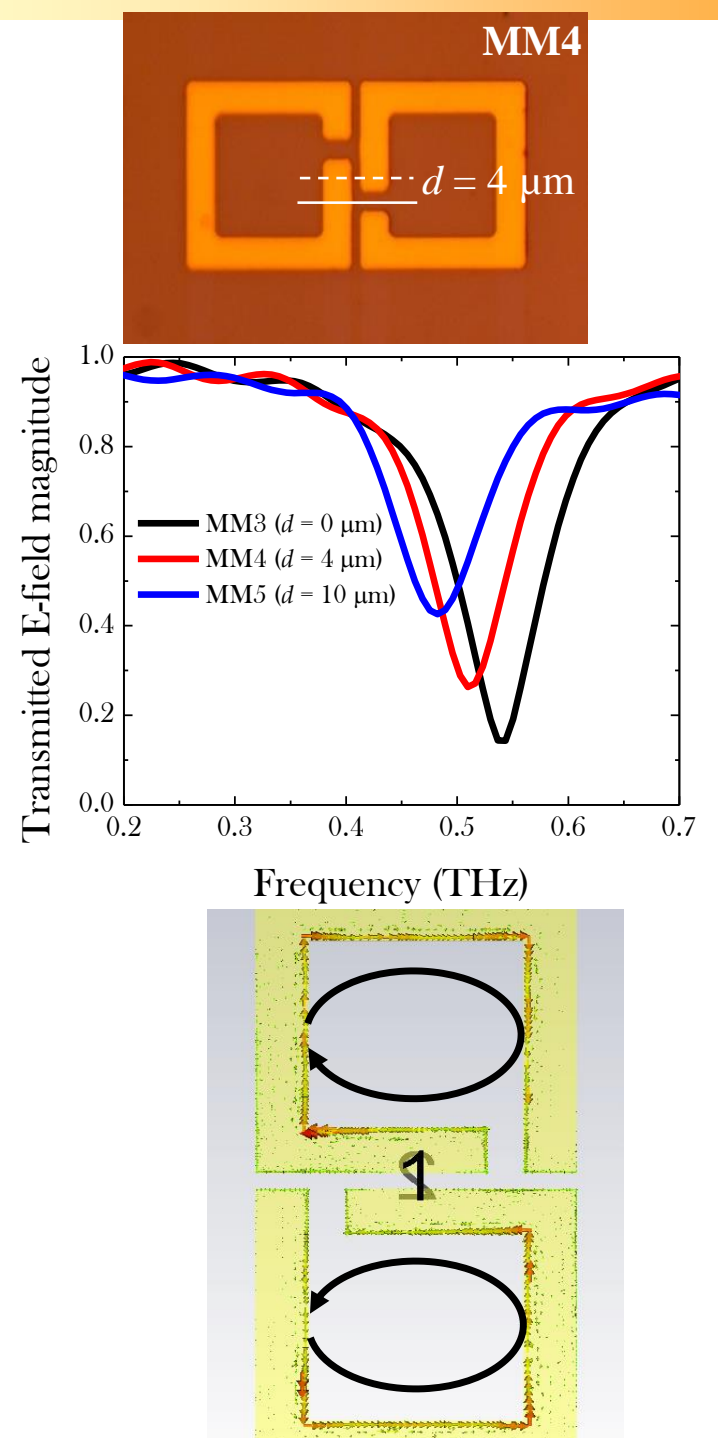

MM3

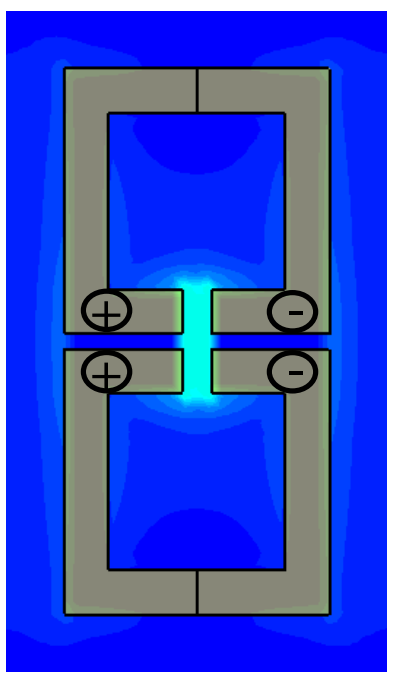

Min.
MM5

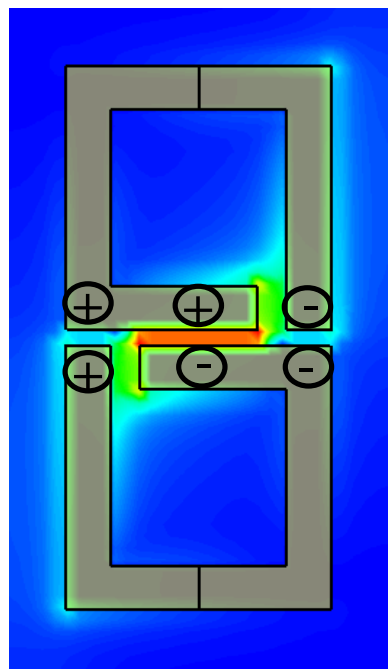

Max.

$>$ Coulomb attraction of opposite charges leads to resonance red shift

$>$ Electric field enhancement in between the SRRs 


\section{Optical switching using bulk semiconductor substrates}
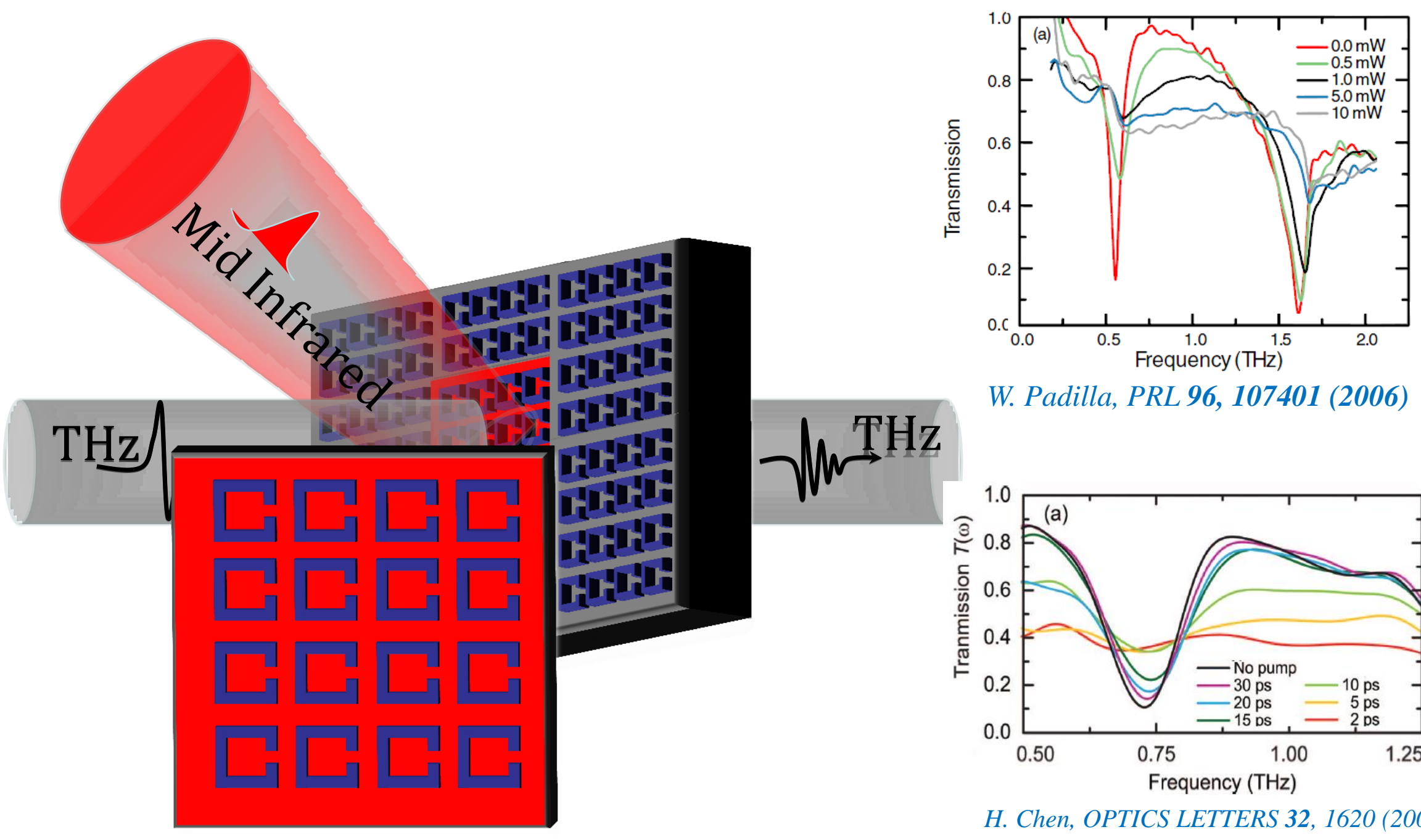

W. Padilla, PRL 96, 107401 (2006)

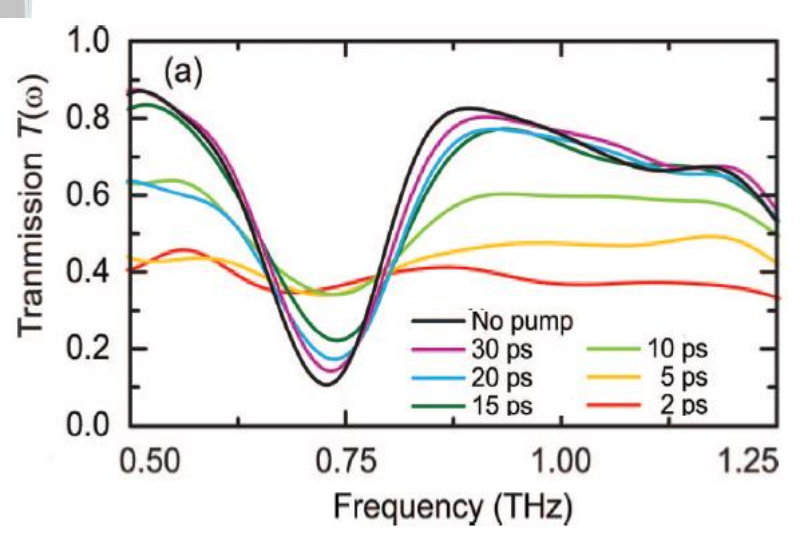

H. Chen, OPTICS LETTERS 32, 1620 (2007) 


\section{Excitation of odd or even resonances in SRR}

SRR odd mode
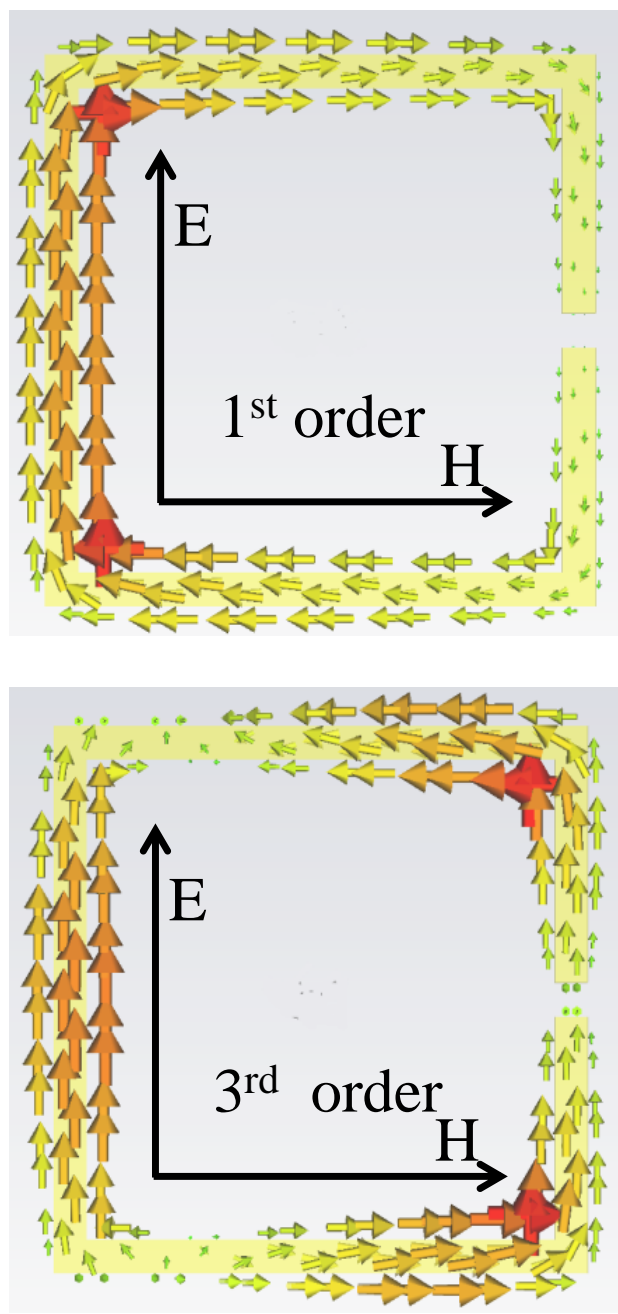

SRR even mode
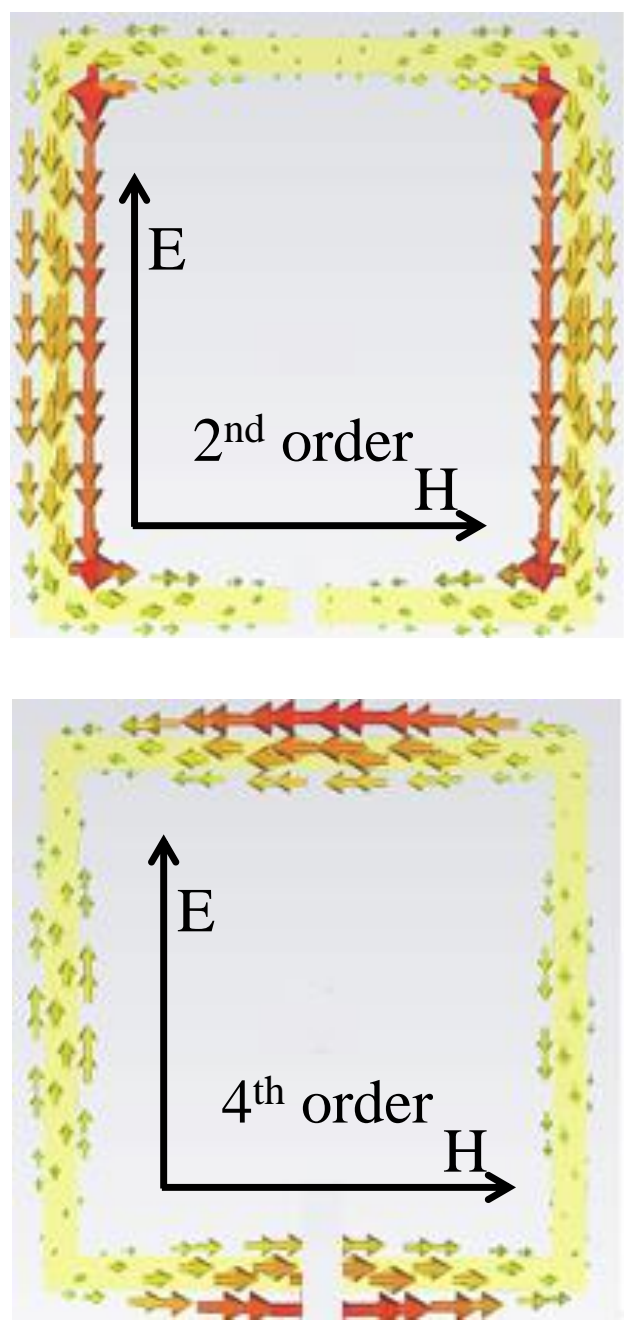

CSR even mode
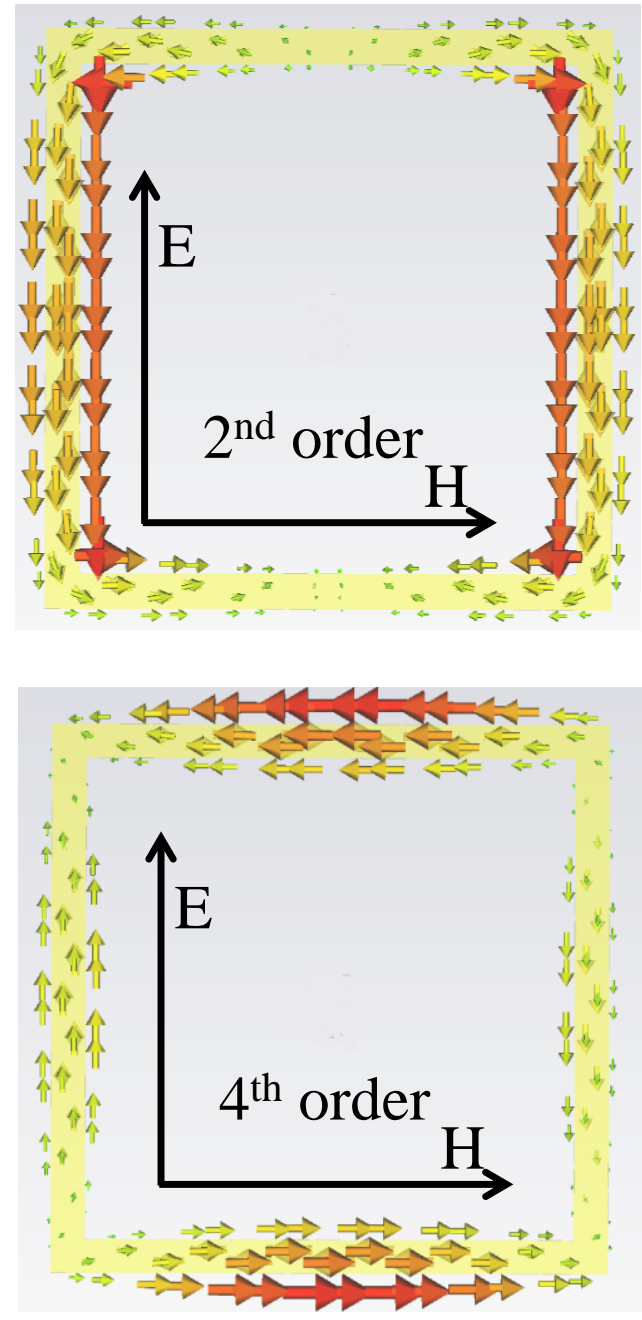


\section{Optically reconfigurable metasurface}

Sample before photoexcitation

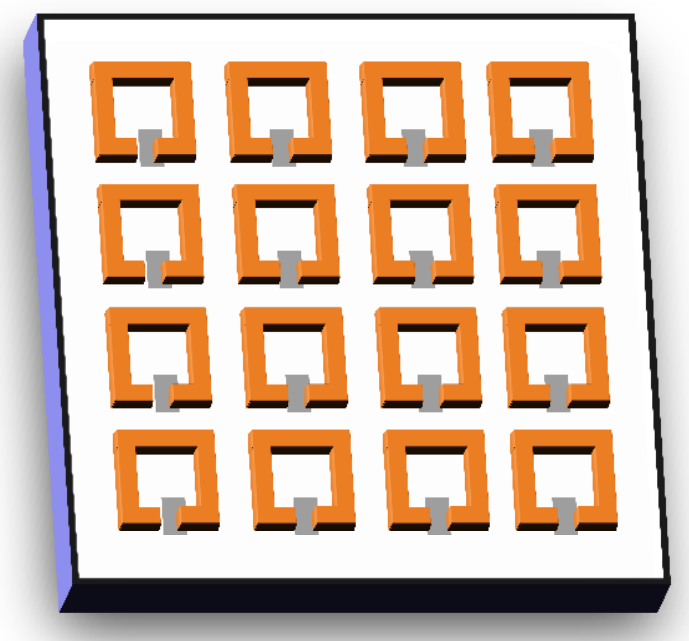

Si island becomes conductive

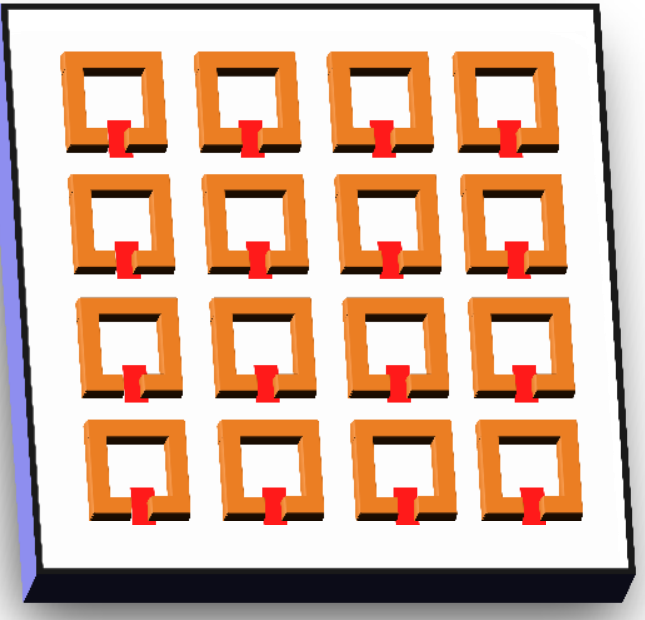

$>$ Substrate is 0.5 micron thick silicon film on sapphire substrate (SOS)

$>$ Silicon islands are small and have unnoticeable effect on $\mathrm{THz}$ transmission while not integrated with SRR.

$>$ Photo excitation transforms the SRR to a $\mathrm{CRR}$, therefore, change the resonant conditions.

$>$ Excites only the even mode resonances

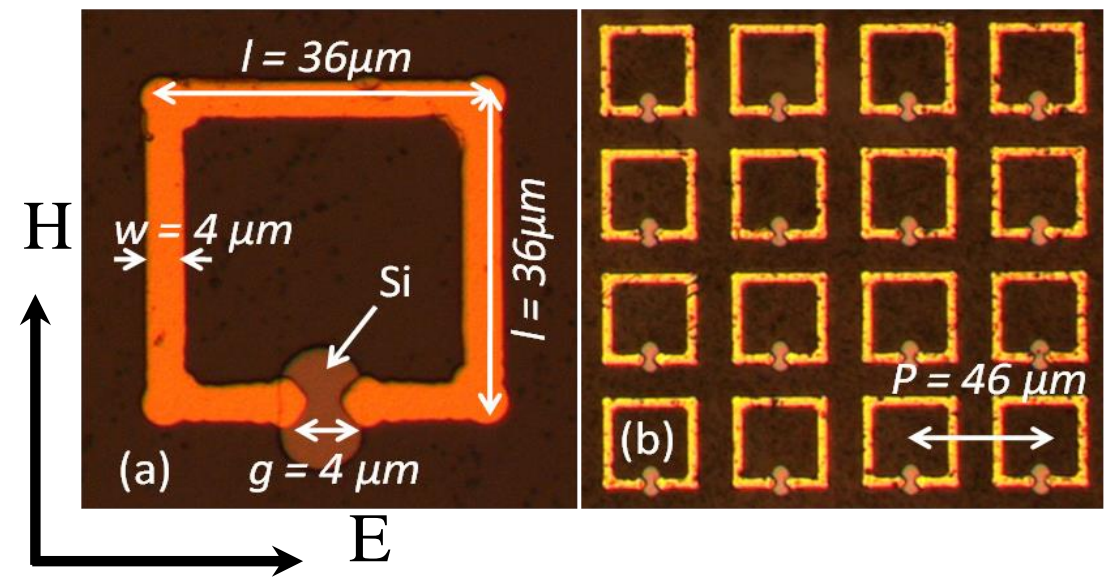

D. Chowdhury, et. al. Appl. Phys. Lett. 99, 231101 (2011) 


\section{Amplitude and phase modulation with optical excitation}

w/o optical excitation

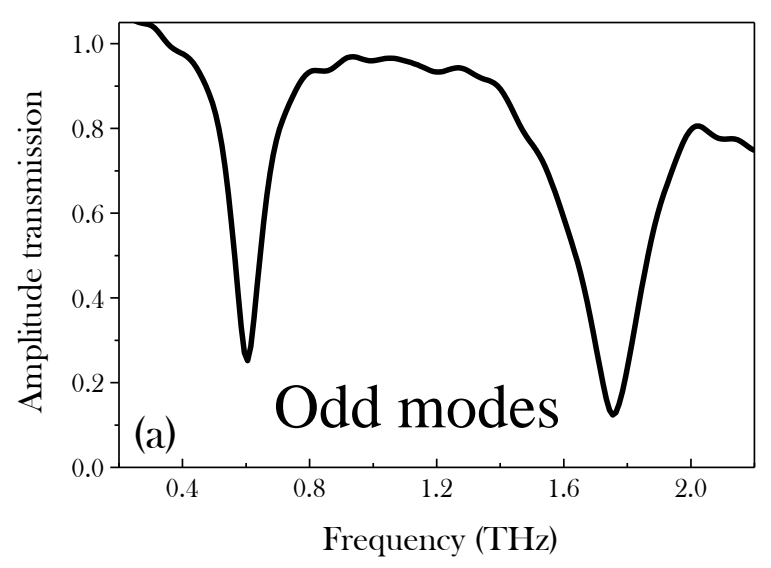

With optical excitation
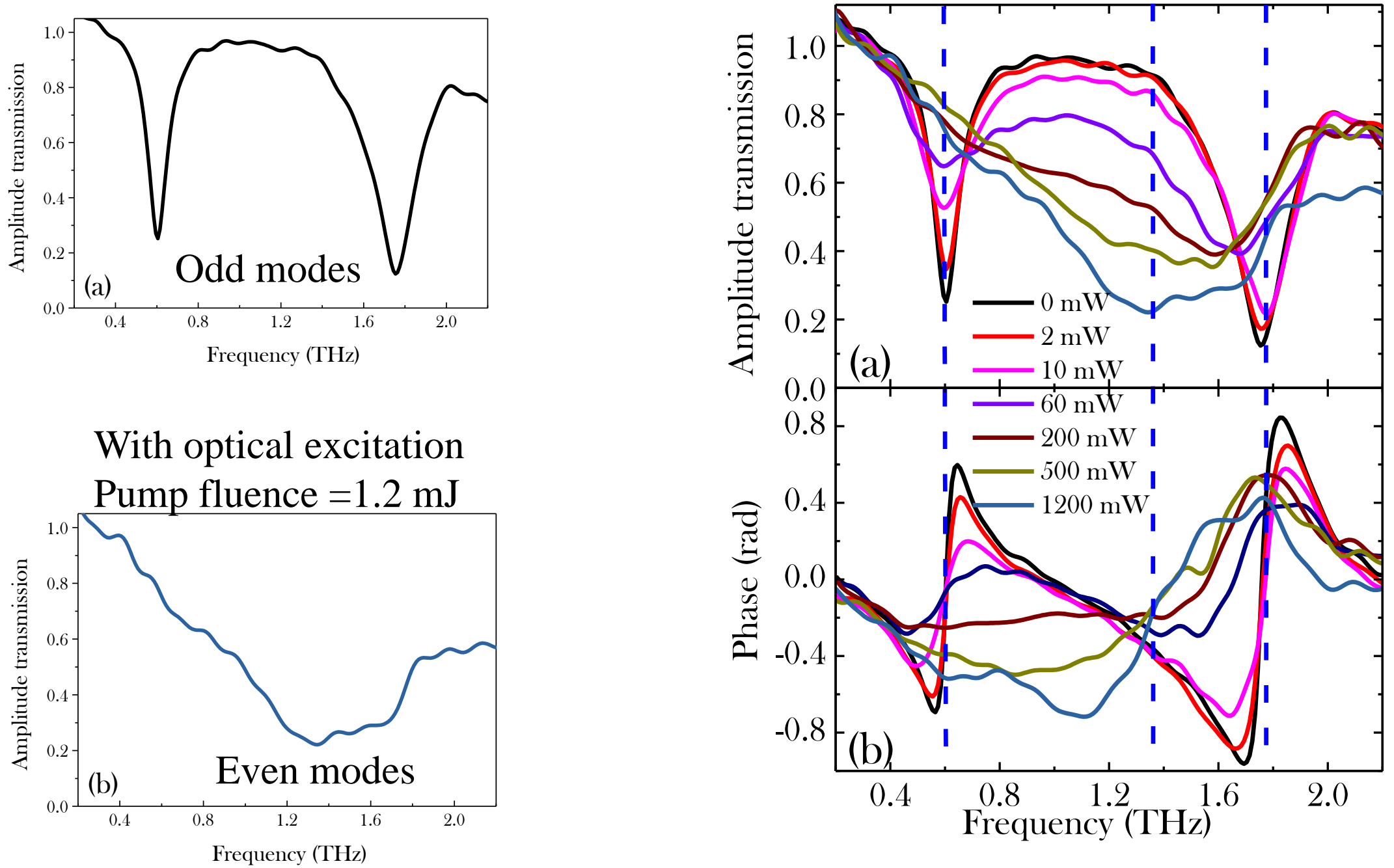


\section{Amplitude and phase modulations due to optical excitation}
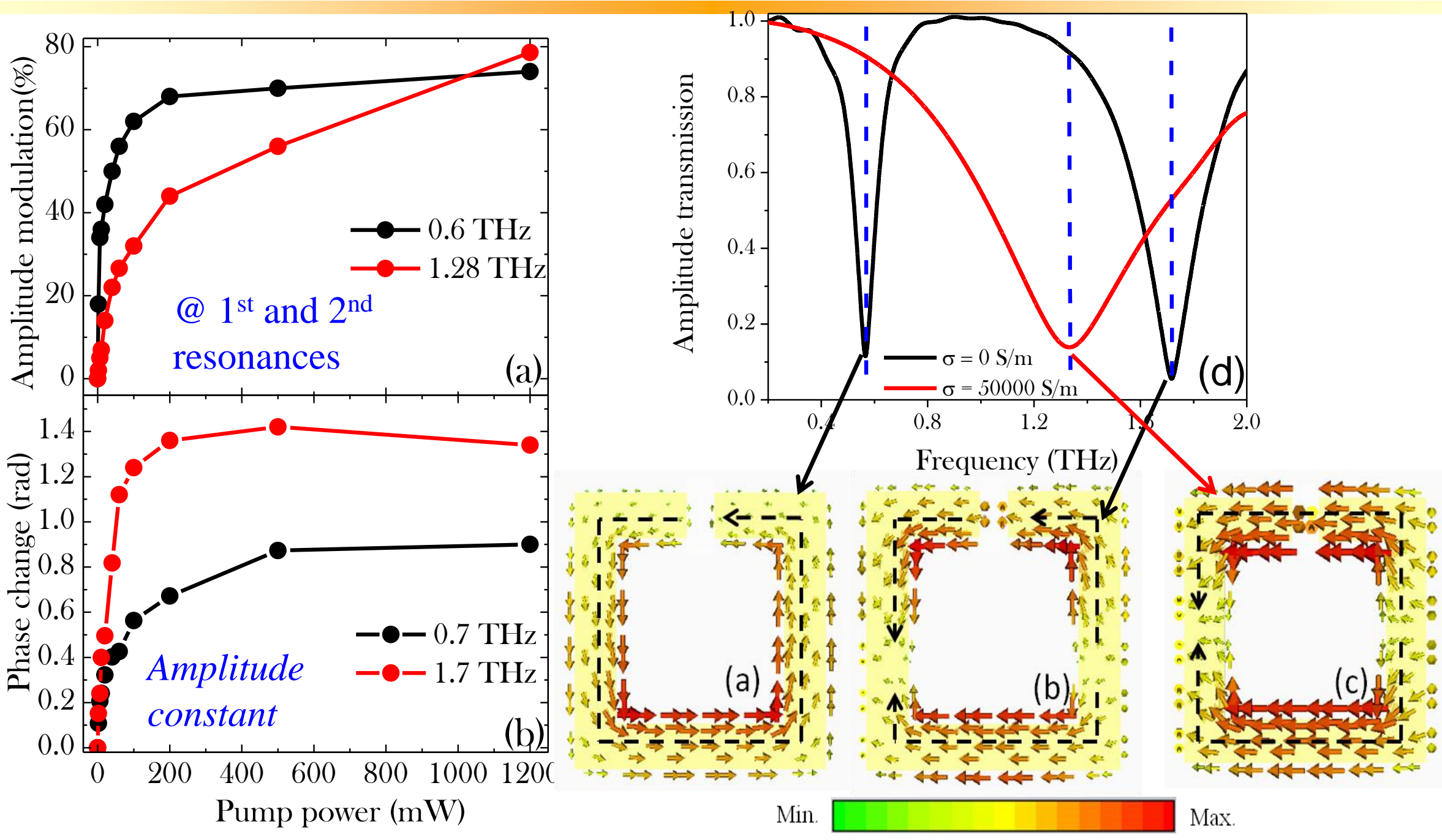


\section{Optically controlled frequency modulation}

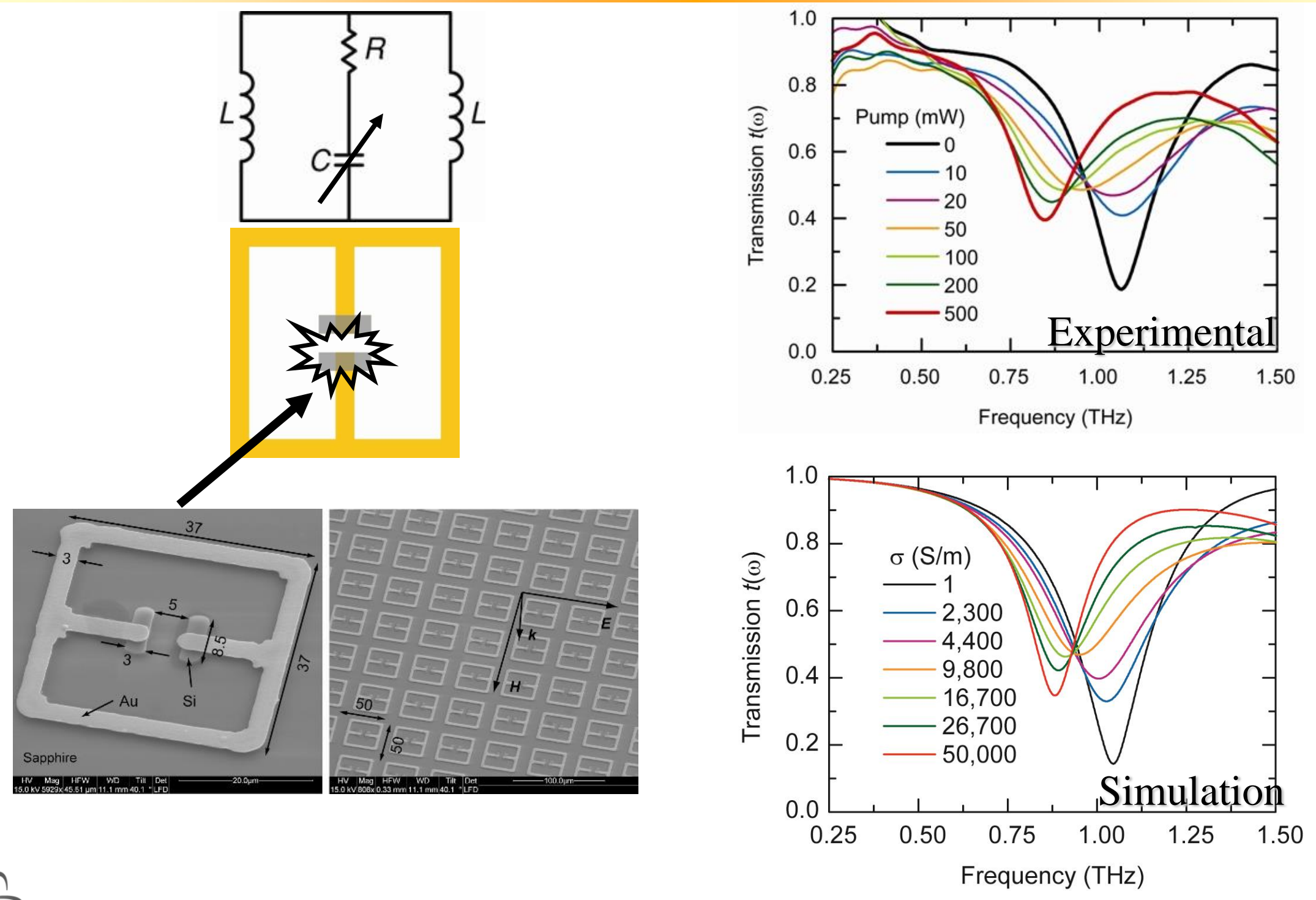

Chen et al., Nature Photon. 2, 295 (2008). 


\section{Static manipulation of coupling between resonators}

(a)

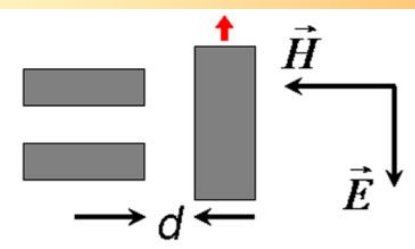

(b)

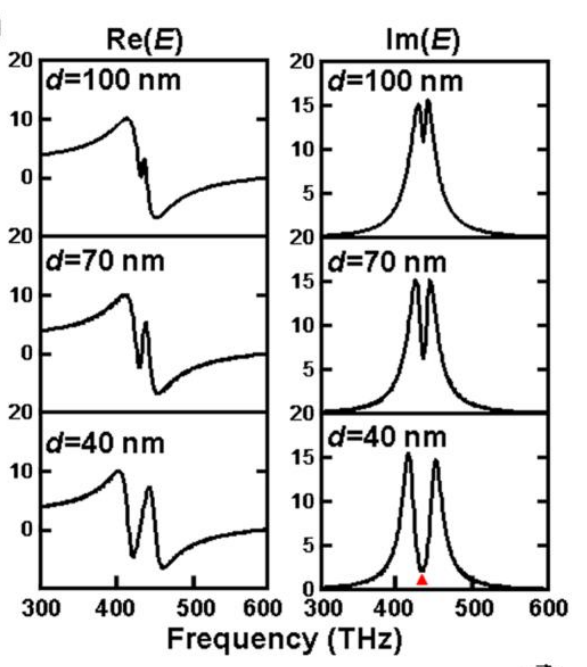

(c)

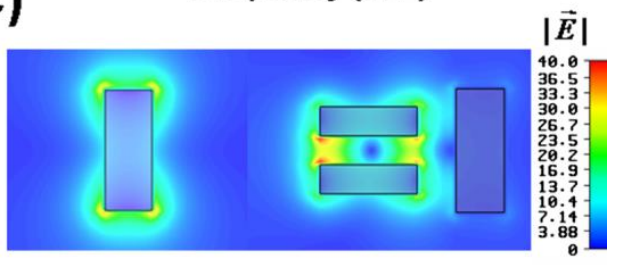

$S$ Zhang et al.

Phys. Rev. Lett., 101, 047401 (2008)

$>$ Plasmonic coupling between bright and dark resonance modes

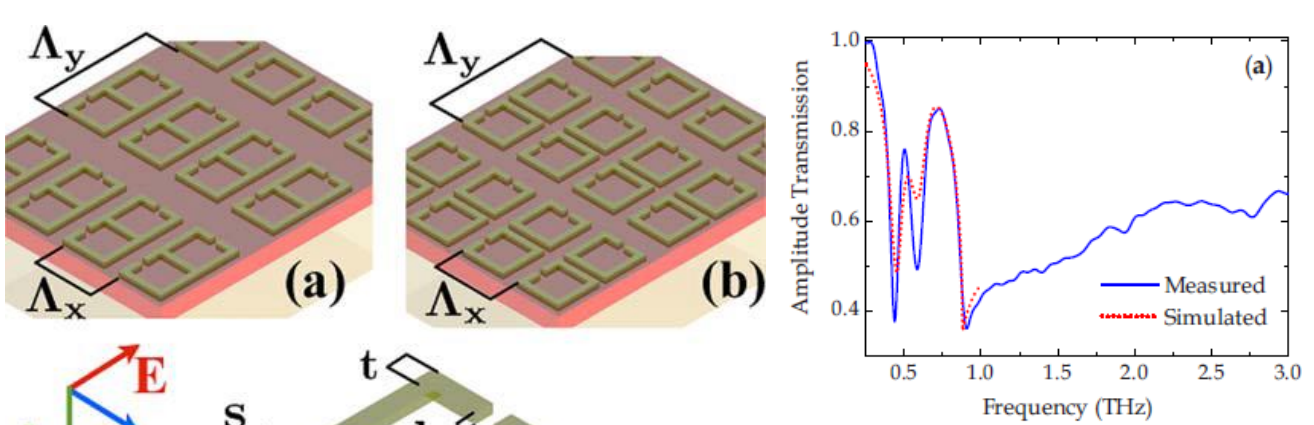

R. Singh et al., Phys. Rev. B 79, 085111 (2009)

Electromagnetic coupling in Metamaterials

$>$ Analogue of electromagnetically induced transparency

Slow light 


\section{Design of the unit metamolecule}

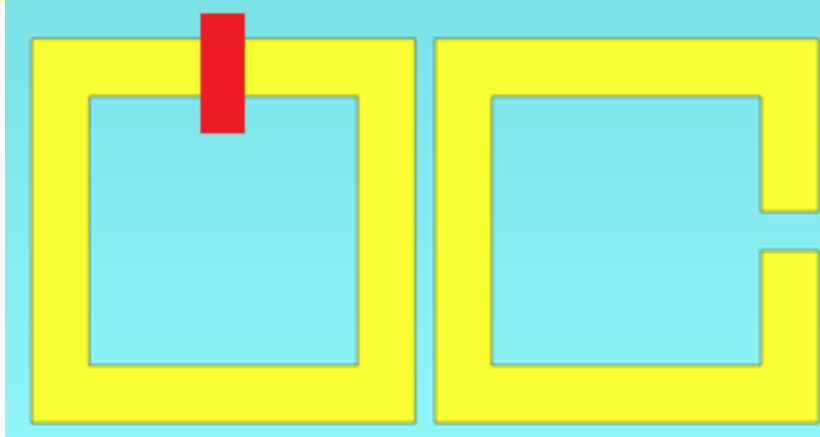

Dark
Conditions for strong coupling:

$\omega_{0(\text { Bright })} \approx \omega_{0 \text { (Dark) }}$

$>$ Separation between Bright and Dark elements must be small

Ultrafast manipulation: dynamically modify $\omega_{0(\text { Dark })}$

Optical excitation by $800 \mathrm{~nm}$ pump

$>$ Ultrafast electron lifetime in Radiation Damaged Silicon (RAD-Si)

$>$ Simultaneous $\mathrm{THz}$ transmission spectroscopy

$>$ At different time delay (ultrafast time scale)

consequence

With different time delay split gap conductivity of dark resonator changes, hence inter resonator magnetic coupling is modified 


\section{Fabricated sample}

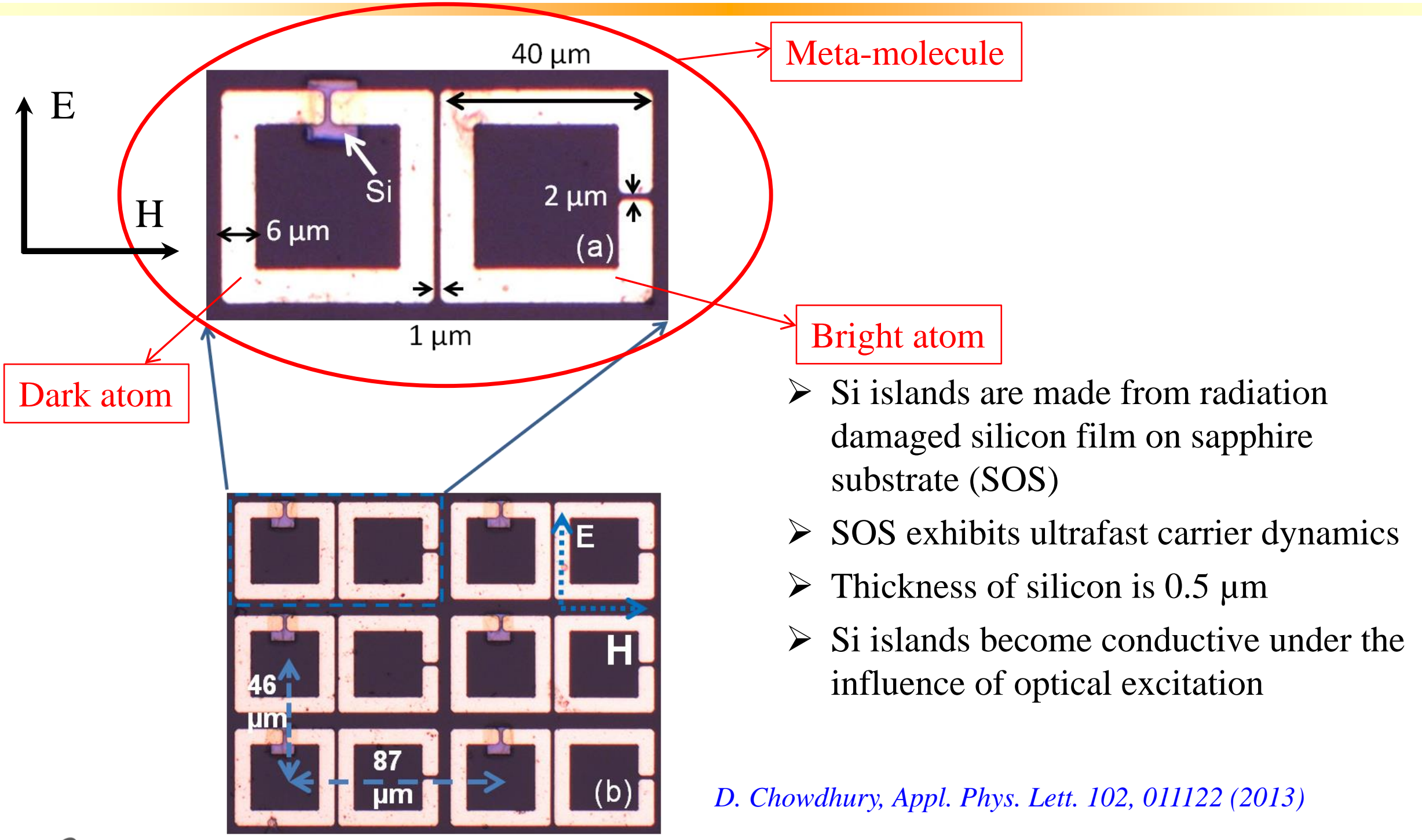




\section{Ultrafast carrier dynamics of RD-SOS}

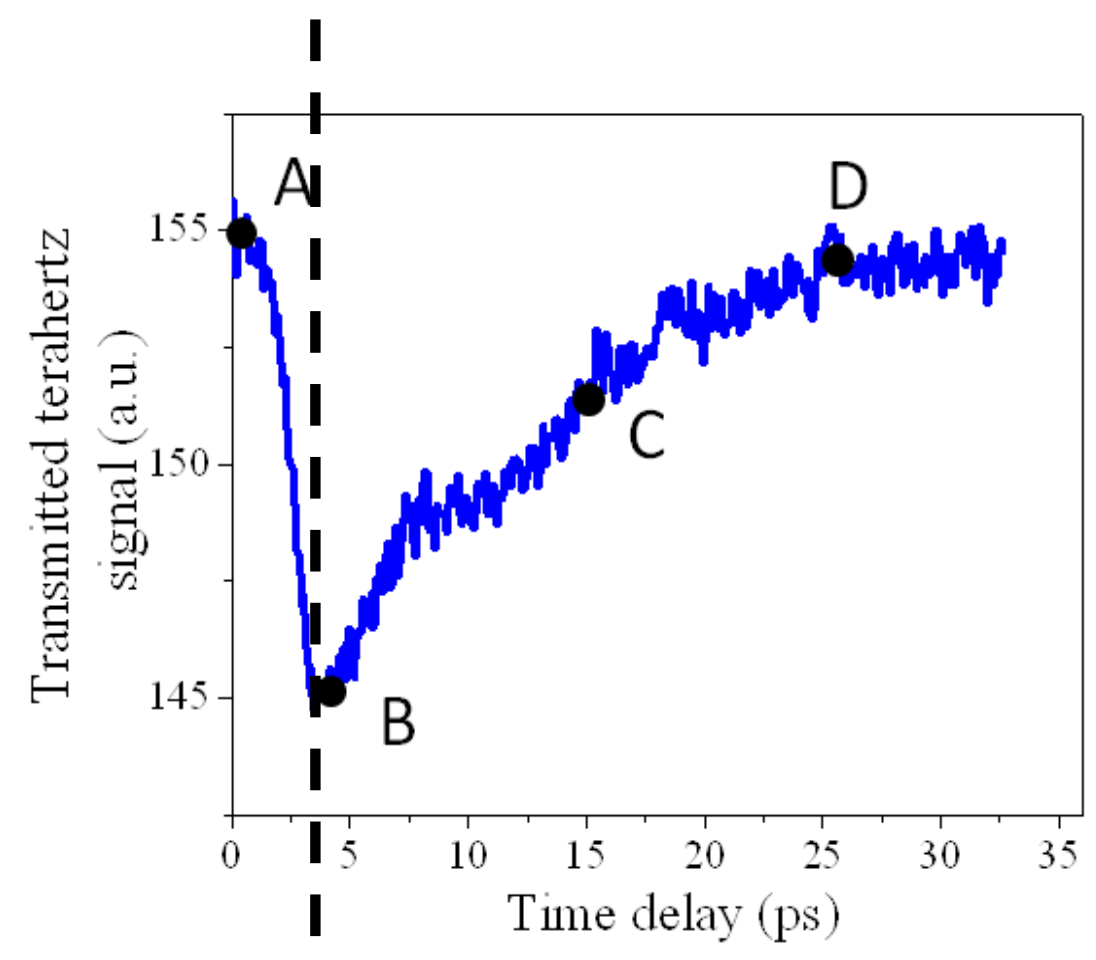

$>$ Conductivity of Si is maximum @ B

$>$ Majority of the carriers recombine @ C

$>$ Si becomes nonconductive again @ D

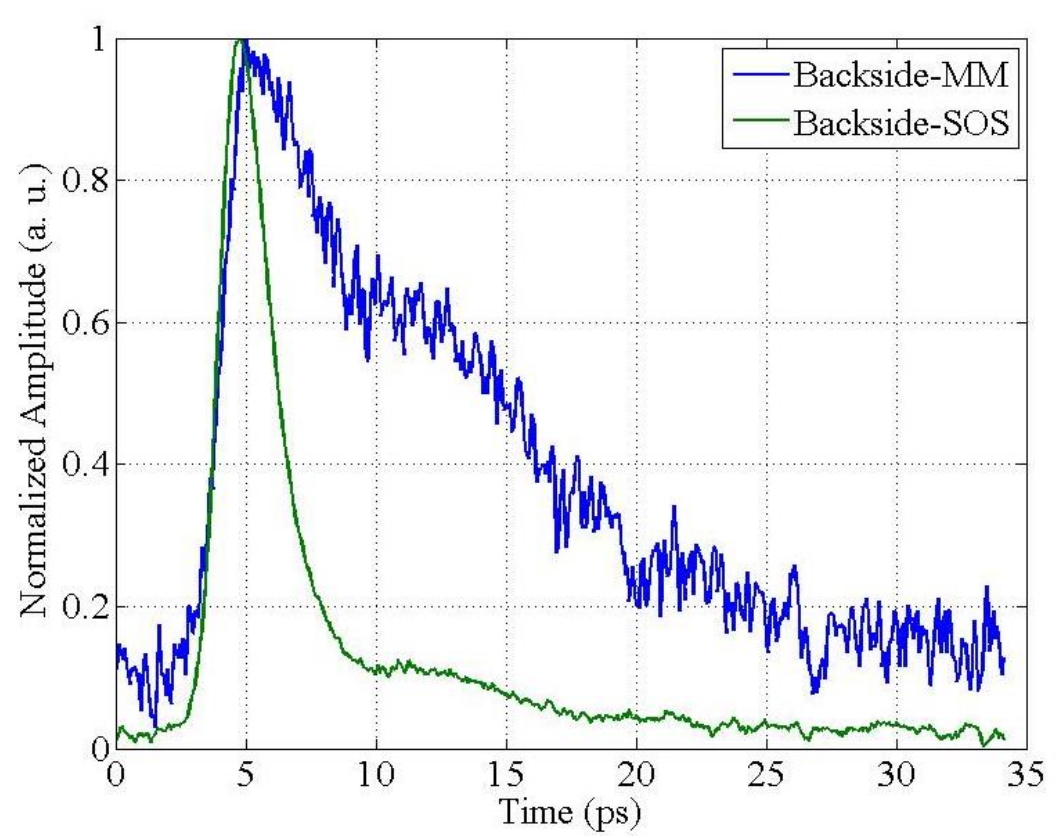

Carrier life times exhibit different dynamics with and without SRR structures

D. Chowdhury, Appl. Phys. Lett. 102, 011122 (2013) 


\section{Amplitude transmission at various time delay}
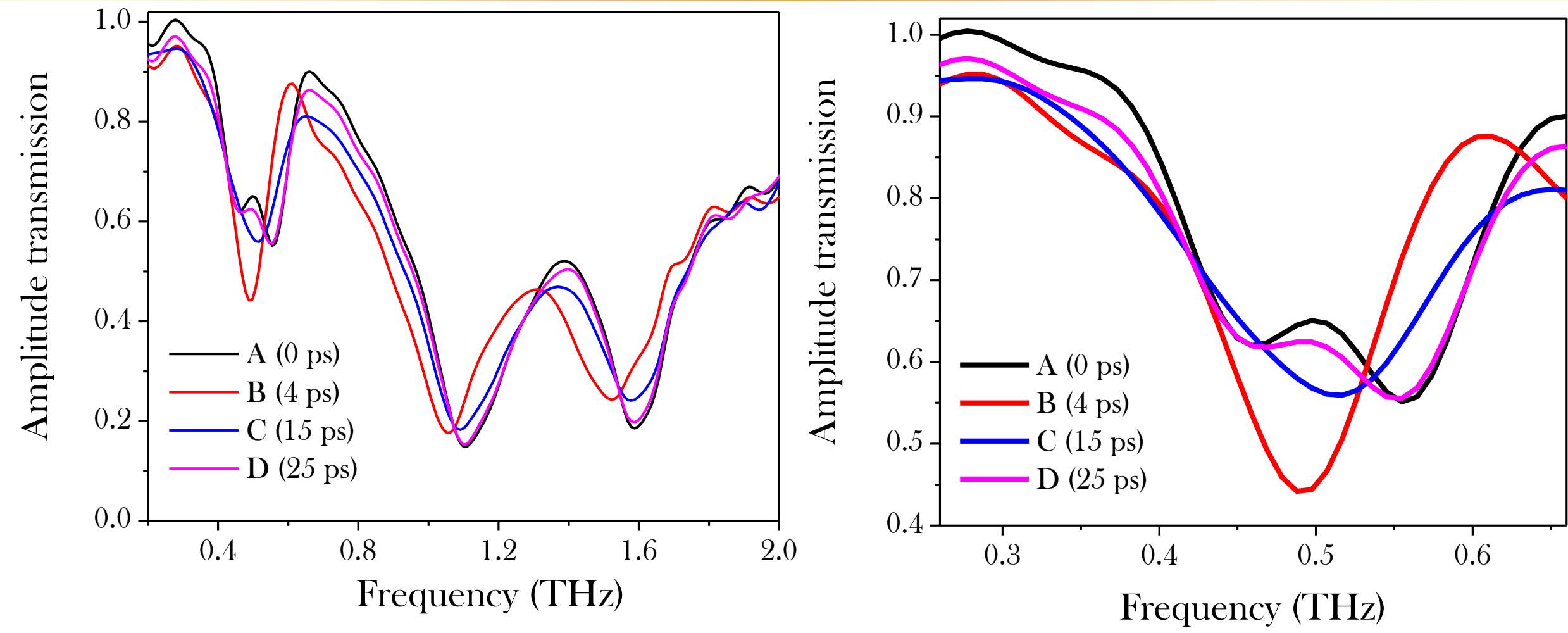

Before optical pump pulse arrive we observe strong coupling

$>$ At point B Si becomes conductive, dark SRRs act like CRR, and the system becomes an uncoupled system - manifested by a single resonance peak

$>$ With time Si becomes nonconductive and the system restores the coupling nature $>$ Complete recovery takes $\sim 25 \mathrm{ps}$

D. Chowdhury, Appl. Phys. Lett. 102, 011122 (2013) 


\section{CST Simulation with different conductivities of silicon}

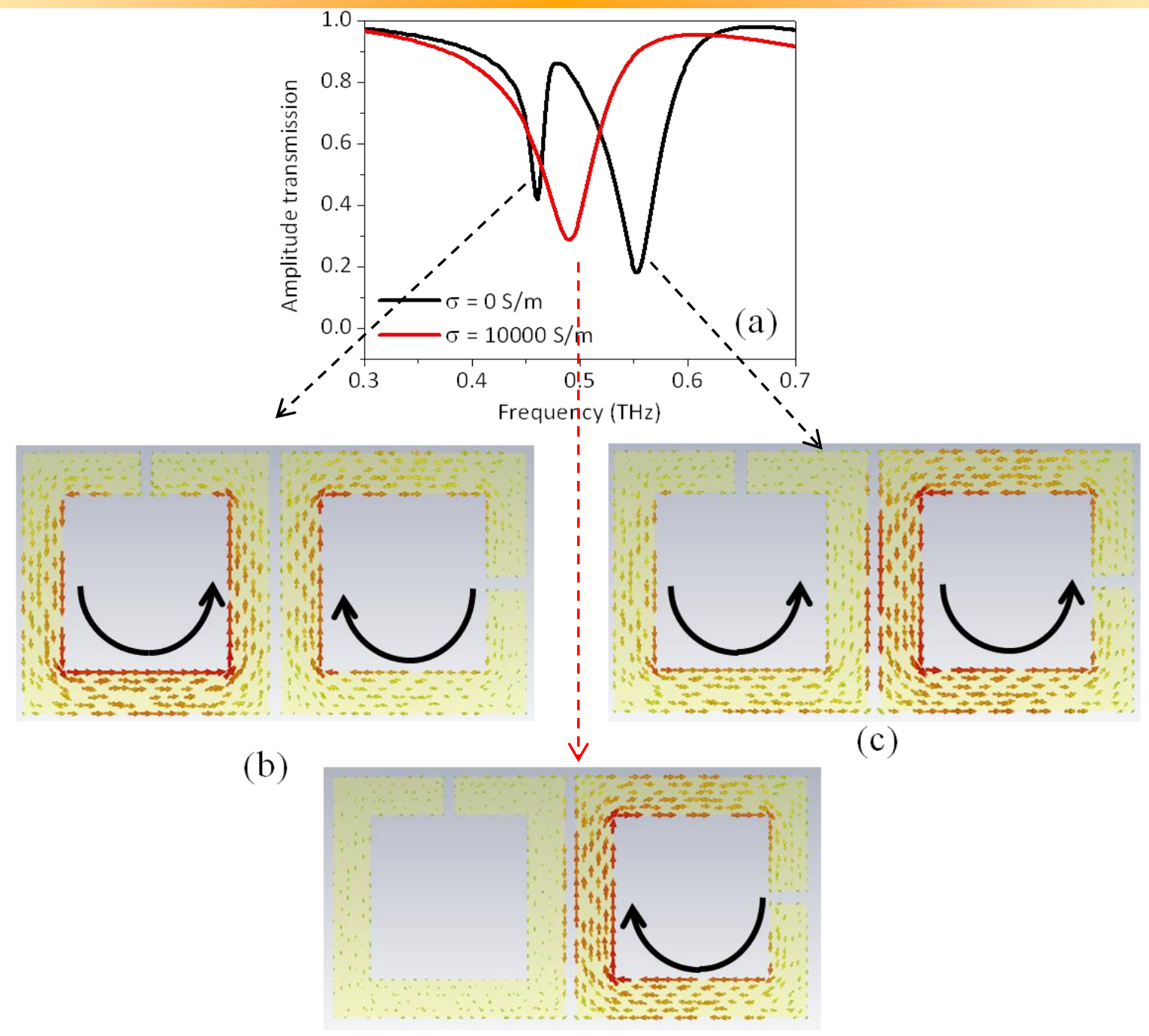

(d) 


\section{Electrically Controlled Modulation}

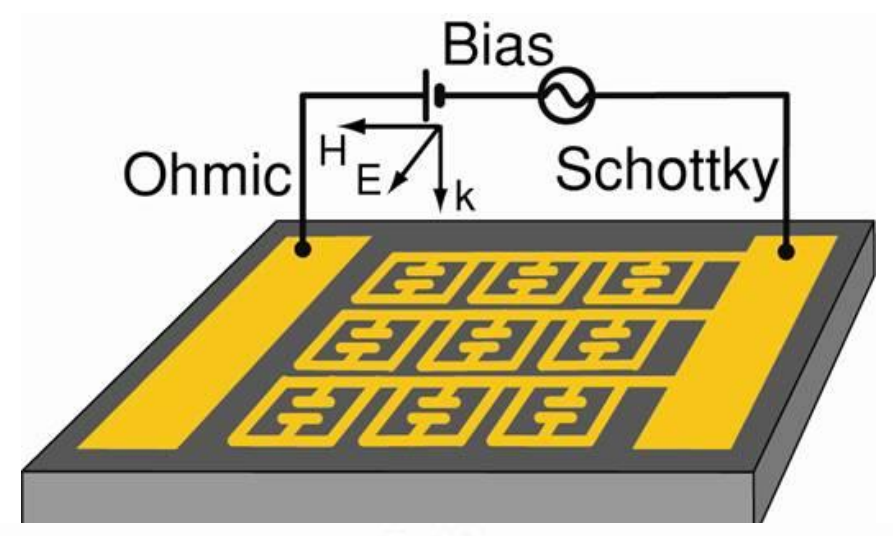

Split gap
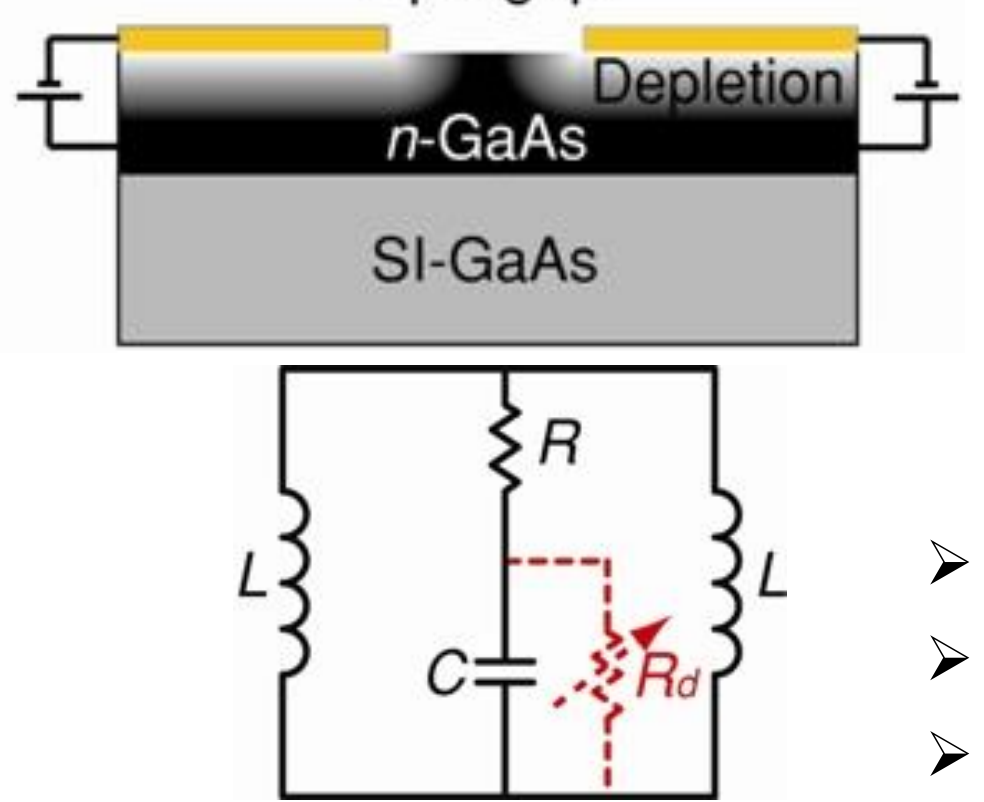

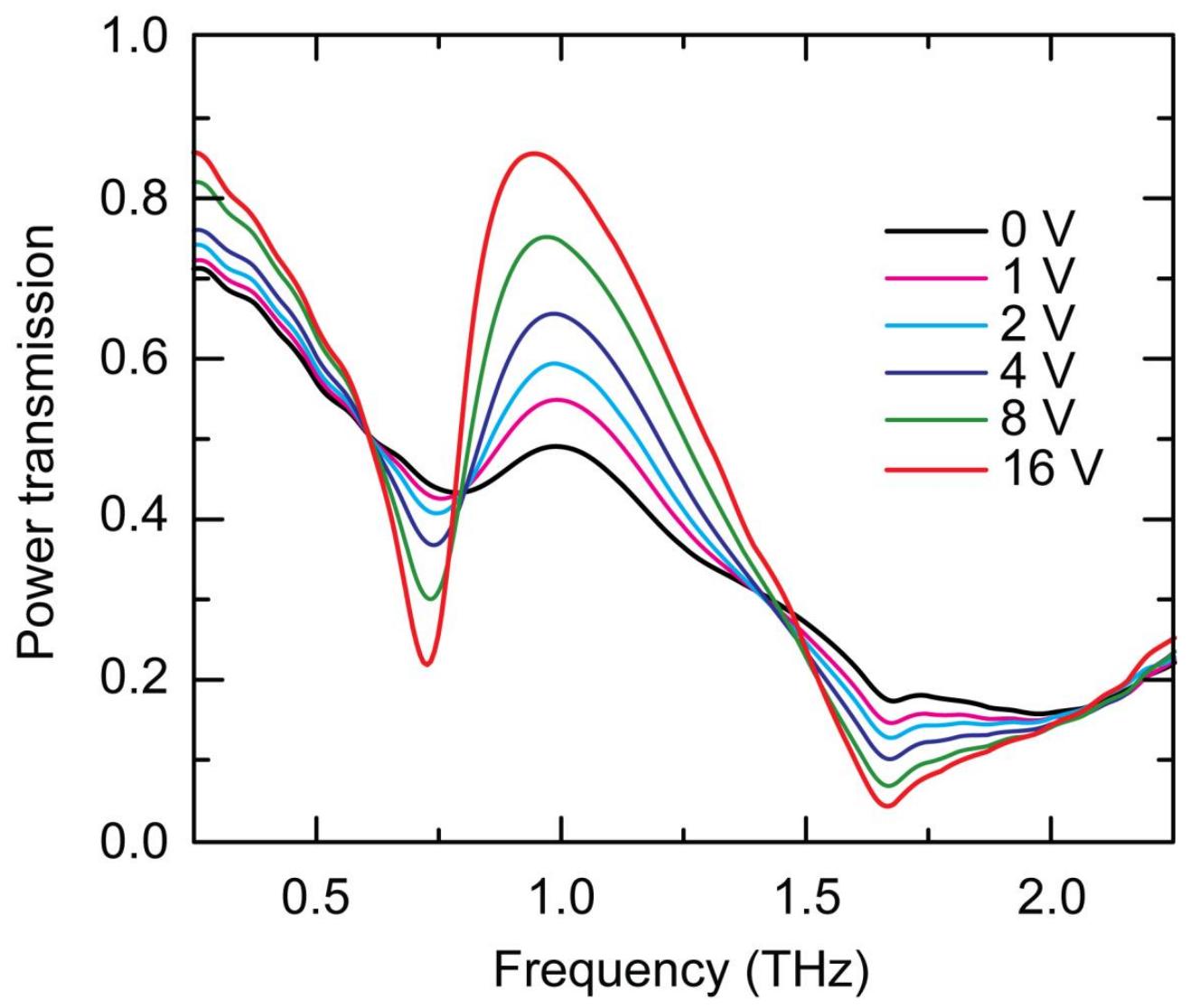

$>$ Power modulation depth: $\Delta T / T=T_{0 \mathrm{~V}}-T_{16 \mathrm{~V}} / T_{0 \mathrm{~V}}=5$

$>$ Modulation up to $2 \mathrm{MHz}$ demonstrated

$>$ Room Temperature Operation 


\section{Solid-state terahertz modulator}
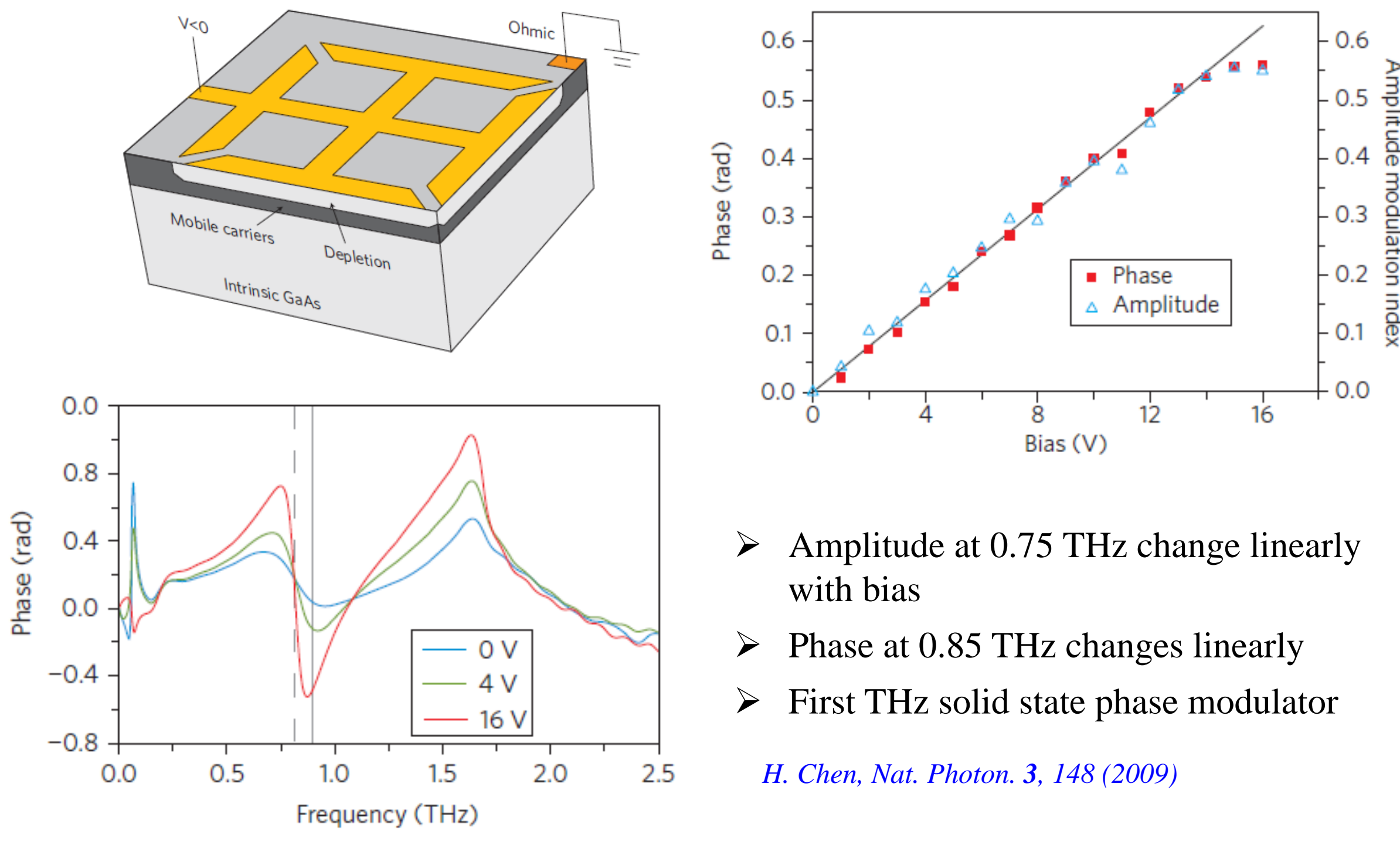

> Amplitude at $0.75 \mathrm{THz}$ change linearly with bias

> Phase at $0.85 \mathrm{THz}$ changes linearly

$>$ First $\mathrm{THz}$ solid state phase modulator

H. Chen, Nat. Photon. 3, 148 (2009) 


\section{Polarization Converter: Reflection}

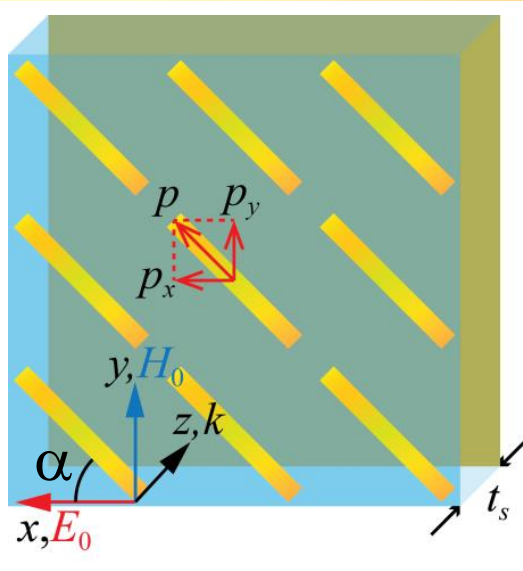

$\square$ Backed with a metal ground plane with appropriate thick dielectric spacer

$\square$ Multiple reflections contain both $\mathrm{x}$ and $\mathrm{y}$ components

$\square$ The co-polarized multiple reflections interfere destructively, leading to minimal co-polarized reflection

$\square$ The cross-polarized multiple reflections interfere constructively, leading to high cross-polarized reflection
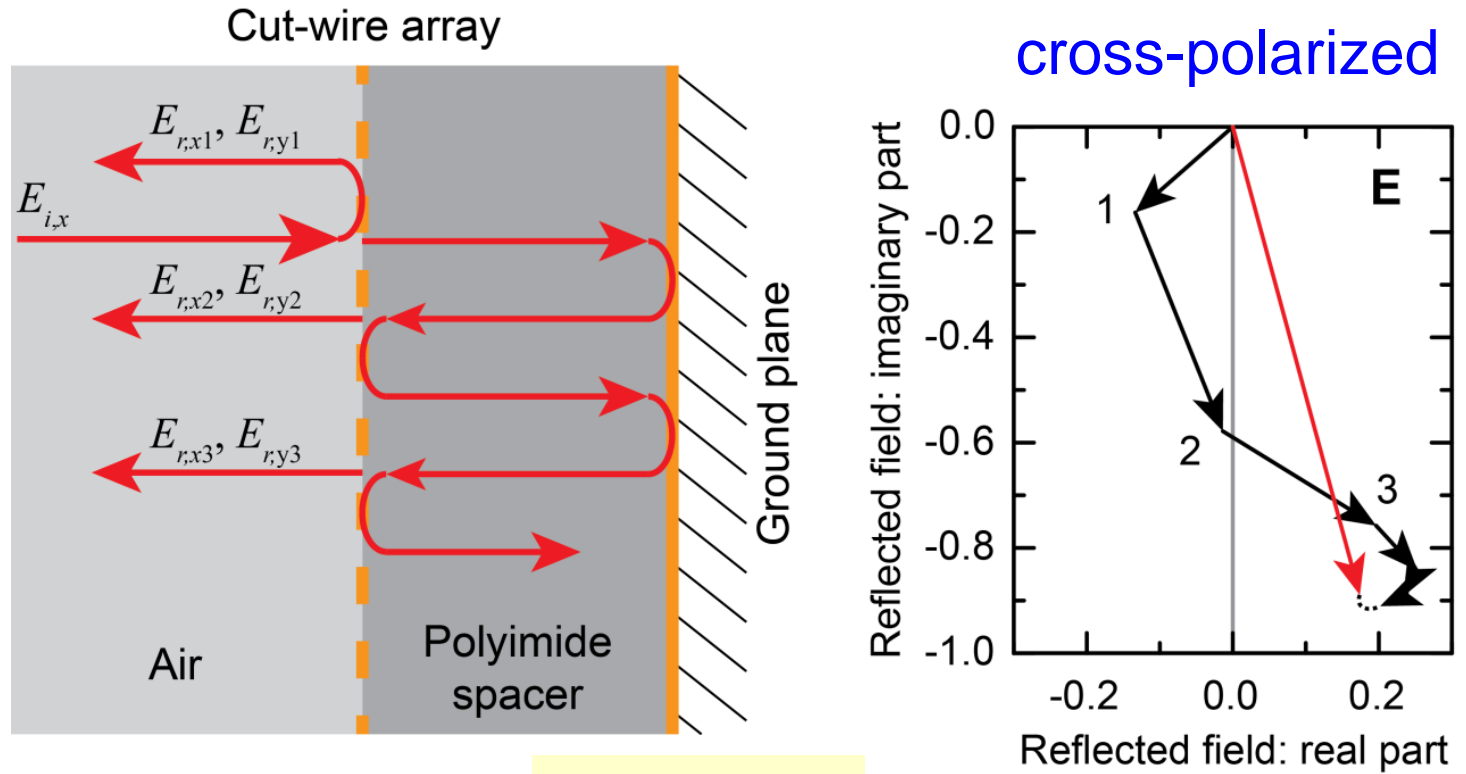

Grady et al., Science 340, 1304 (2013).

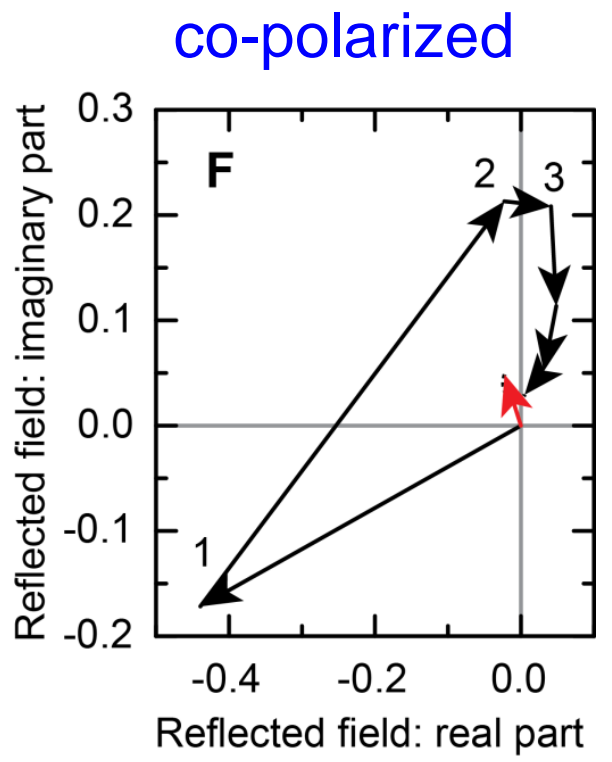




\section{Metamaterial Polarization Converter: Reflection}

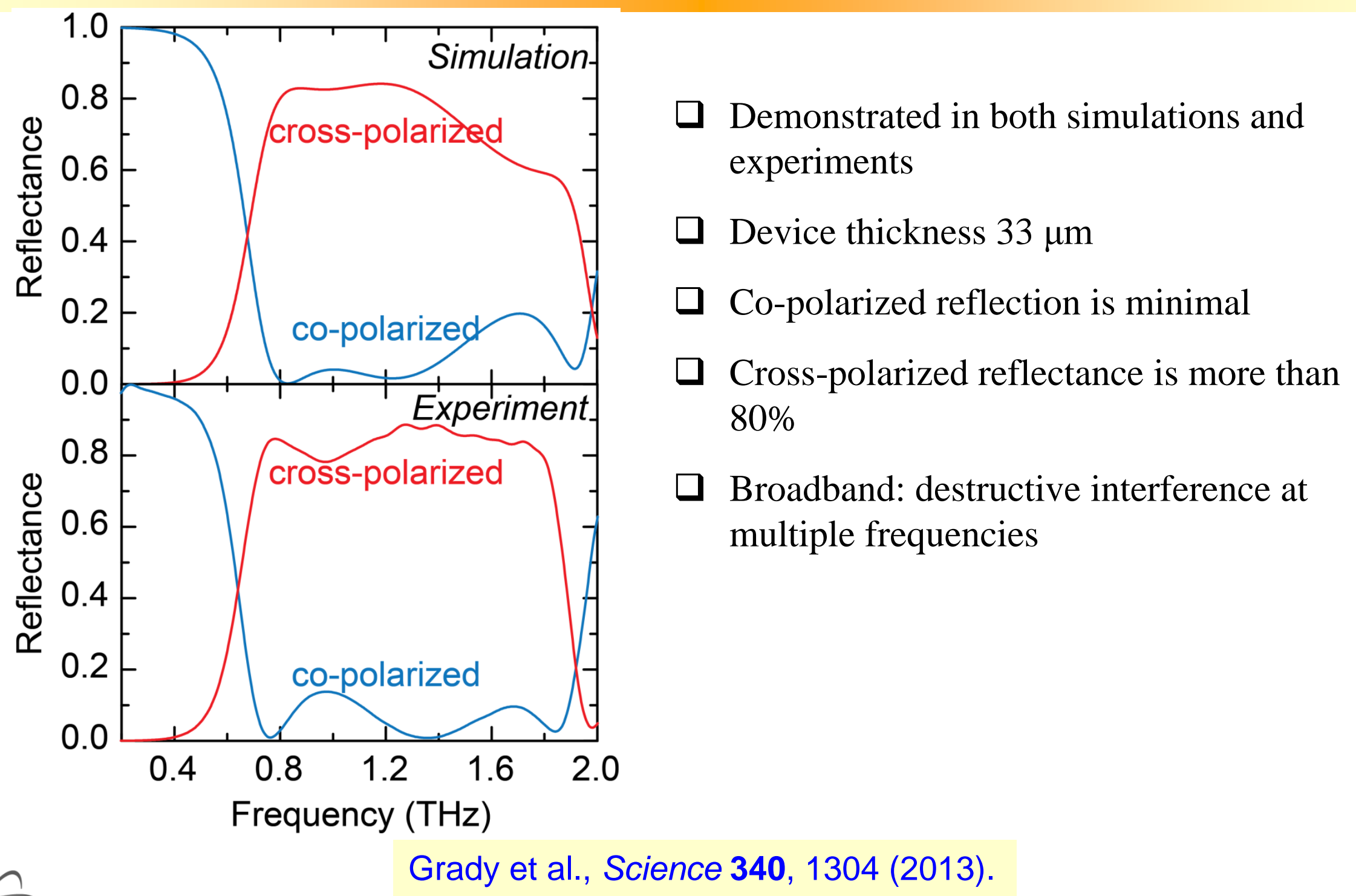




\section{Polarization Converter: Transmission}

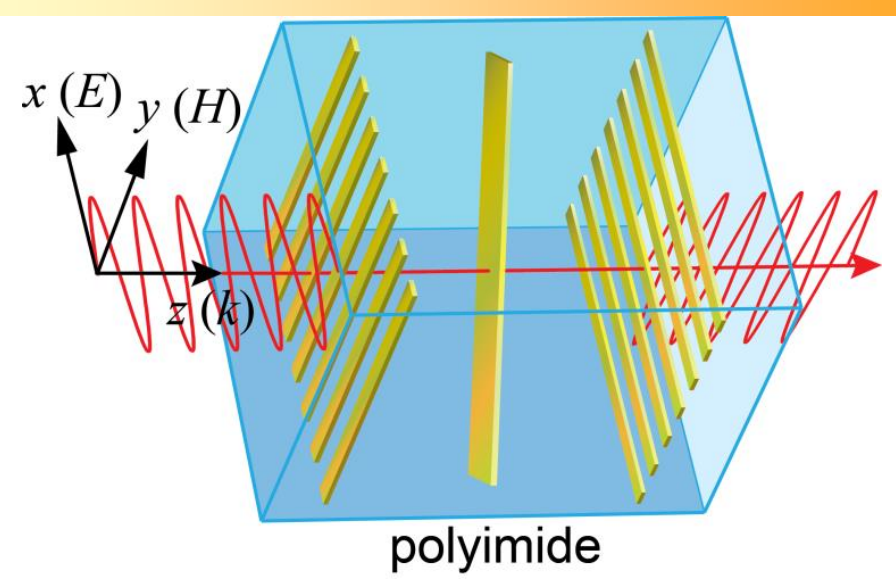

- The gratings are equivalent to ground plane for electric field polarized along the grating lines.

The back grating prevents co-polarized light from transmitting through

The front grating prevents the converted crosspolarized light from reflecting back

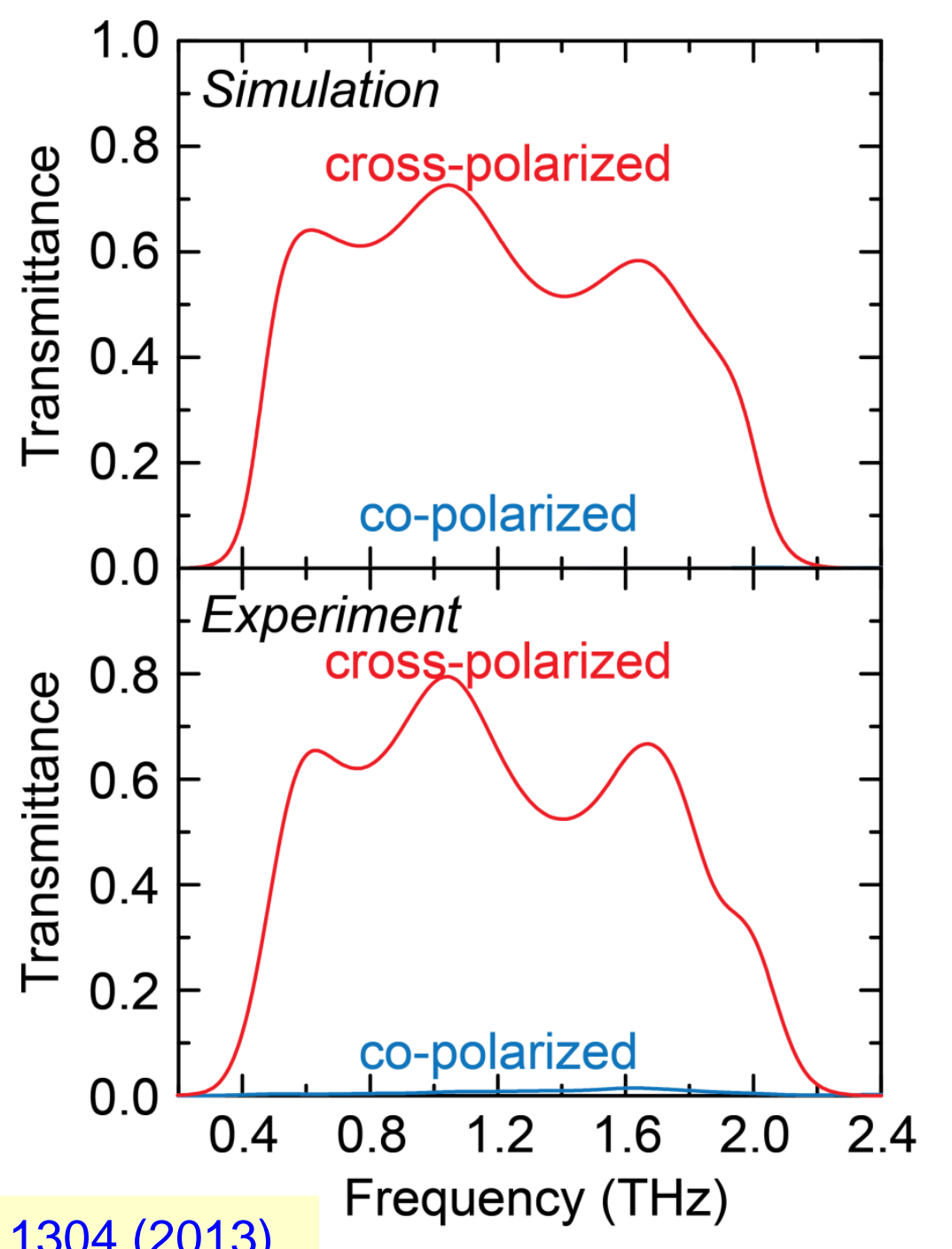

Grady et al., Science 340, 1304 (2013). 


\section{Anomalous Refraction: Generalized Snell's Law}

$\begin{array}{llllllll}1 & 2 & 3 & 4 & 5 & 6 & 7 & 8\end{array}$ $\square \square L \vee \square \square \square$
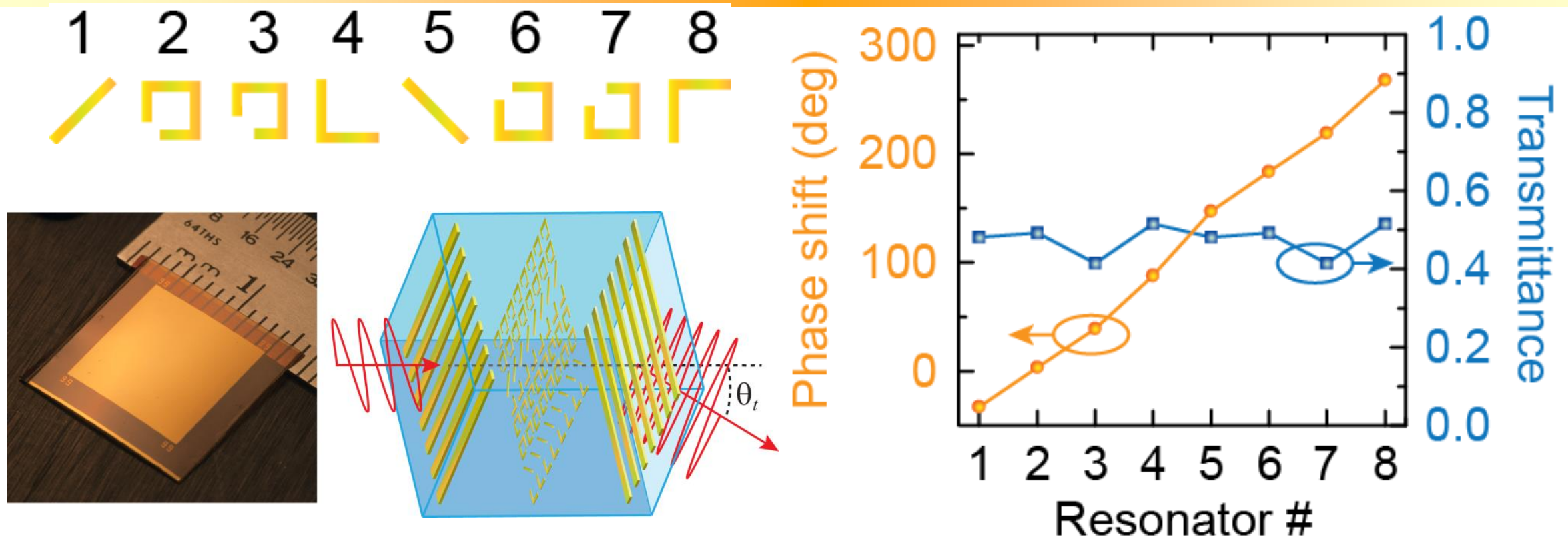

$\square$ Each individual element can be used in $\sin \theta_{t}-\sin \theta_{i}=\frac{\lambda_{0}}{2 \pi} \frac{\mathrm{d} \Phi}{\mathrm{d} x}$ polarization converter

$\square$ Conversion efficiency is designed to be largely constant

$\square 8$ elements form the unit cell, with a linear phase shift spanning a $2 \pi$ range

Grady et al., Science 340, 1304 (2013). 


\section{Near-Perfect Anomalous Refraction: Exp.}
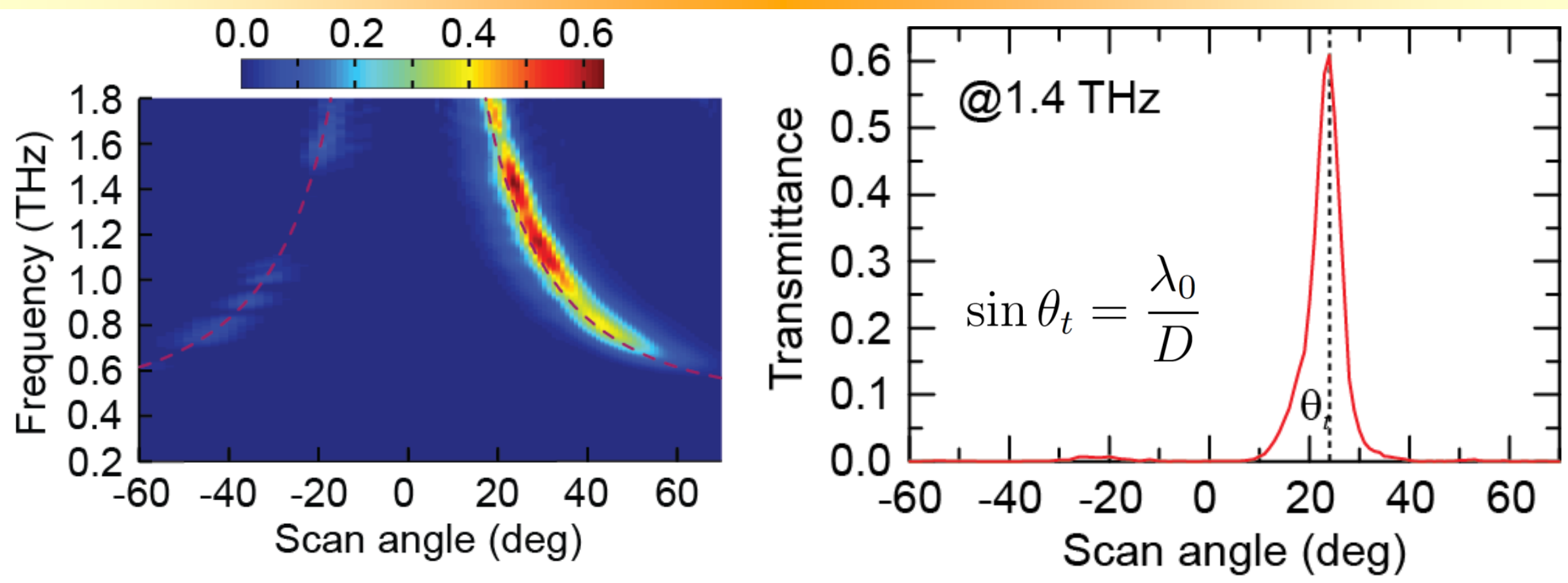

$\square$ At a specific frequency, the "refraction angle" is determined by periodicity

$\square$ At $1.4 \mathrm{THz}$, the anomalous refraction carries $60 \%$ of the incident power

$\square$ Measure the cross-polarized transmission vs. scanning angle

$\square$ Operate over a broad bandwidth

Grady et al., Science 340, 1304 (2013). 


\section{Summary}

$\square$ Engineered metasurfaces at $\mathrm{THz}$ frequencies, Strong resonance response

$\square$ Understanding basic properties with respect to design parameters

$\square$ Investigated the coupling mechanism and the corresponding effect on resonances

$\square$ Actively modulation of THz radiation using optical controls

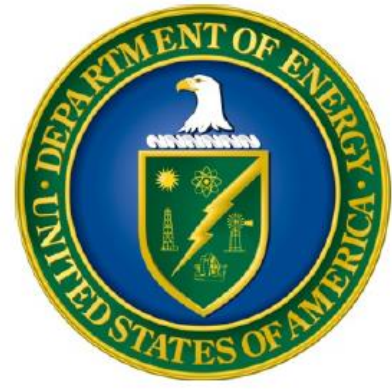

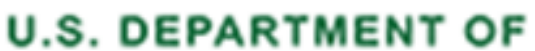
ENERGY
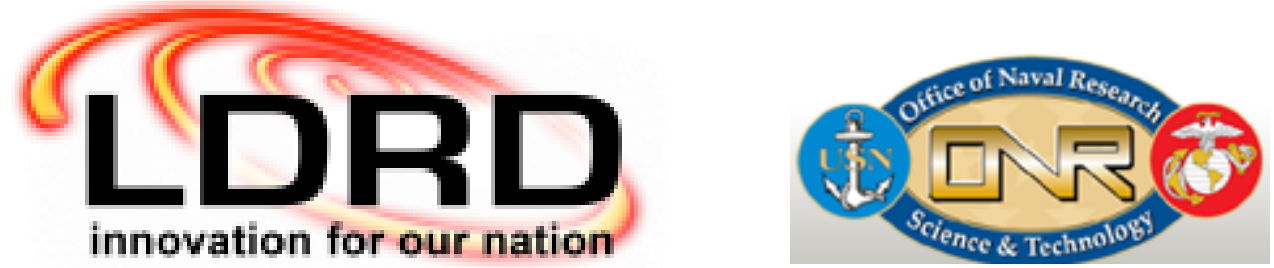

\section{Laboratory Directed Research \& Development}




\section{Electrical Generation of Microwave and THz}

Split Ring Resonator acts as a tank circuit providing feedback and antenna

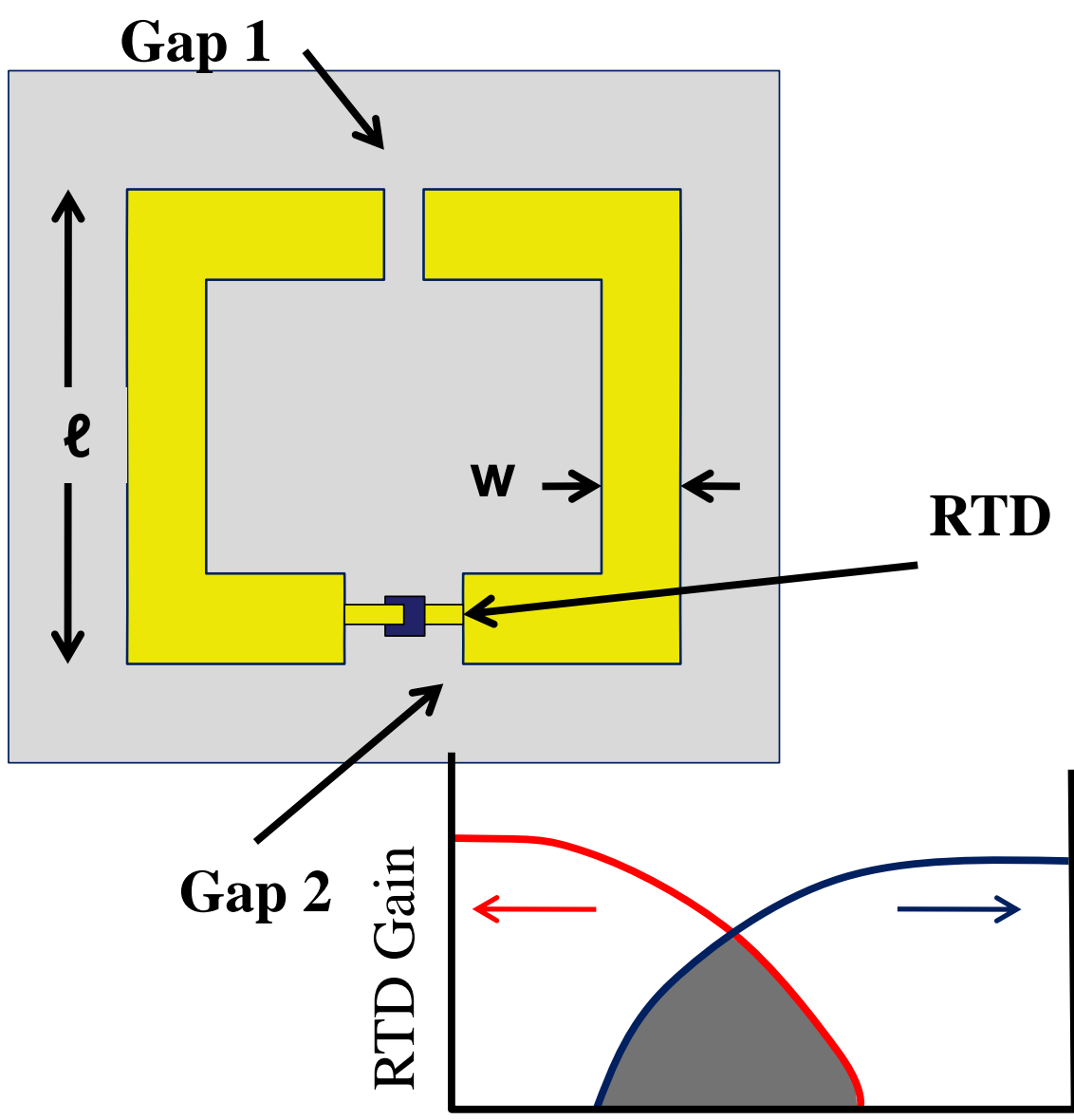

Frequency
Resonant Tunneling Diode (RTD) is active gain element 


\section{Electrical Generation of Microwave and THz}
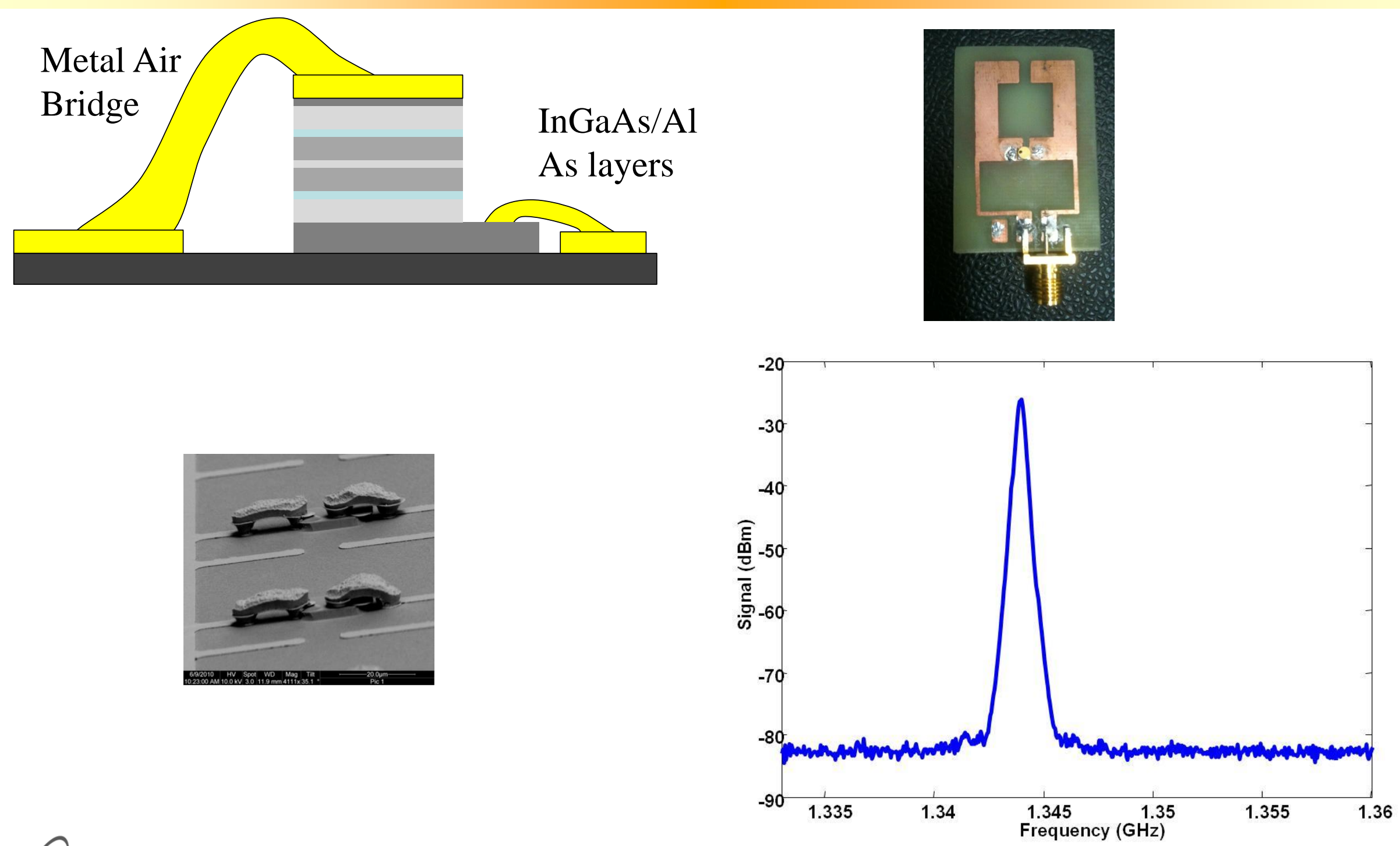


\section{Team and Collaborators}

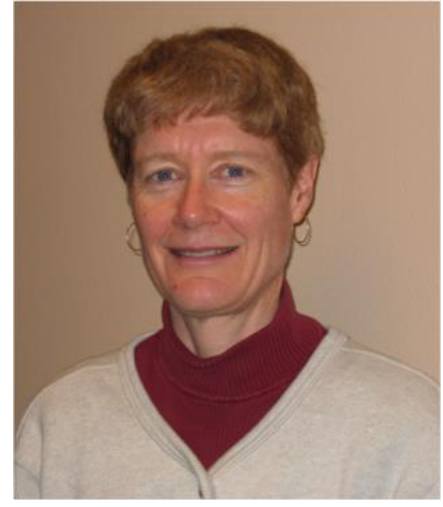

Toni Taylor

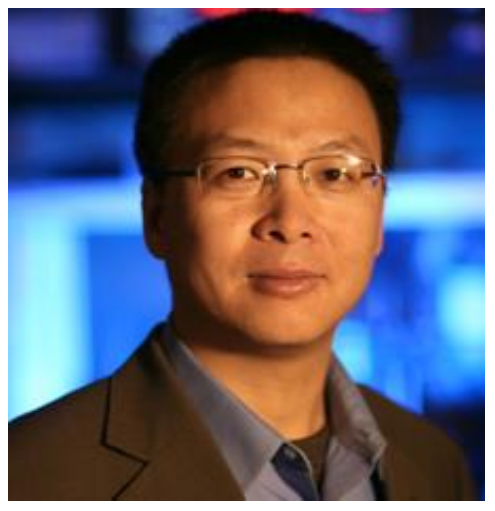

Hou-Tong Chen

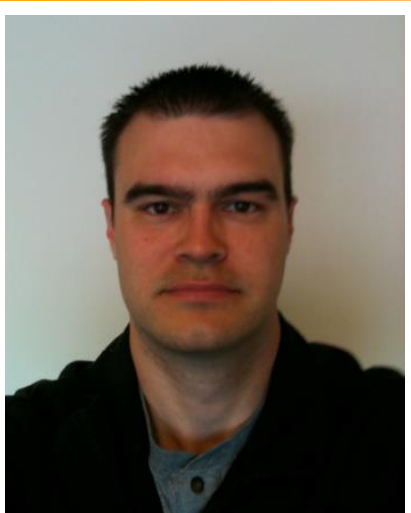

John O’Hara
Matthew Reiten Dibakar Chowdhury

Nathaniel Grady

Suchitra Ramani

LANL: Quanxi Jia, Stuart Trugman, Diego Dalvit, Lawrence Earley, Michael Holloway, Evgenya Smirnova, Dmitry Shchegolkov

Oklahoma State University: Weili Zhang (weili.zhang@okstate.edu)

Nanyang Technological University: Ranjan Singh (RANJANS@ntu.edu.sg)

University of Minnesota: Jeong-Hyun Cho (jcho@umn.edu)

University of Delaware: Josh Zide (zide@udel.edu), and Matthew Doty (doty@udel.edu)

Okinawa Inst. Of Sci. \& Tech., Grad. Univ.: Keshav Dani

University of Utah: Ajay Nahata (doty@udel.edu)

Duke University: David Smith, Nan Jokerst 


\section{My special thanks to}

Professor Dr. Y. Haider

Professor Dr. Monowar Abedin 


\section{Strongly coupled planar metasurface}

$>$ Meta-atom based meta-molecule

$>$ Meta-atom can be bright or optically active for certain polarization or dark

$>$ Strong coupling can be realized through concentric configuration
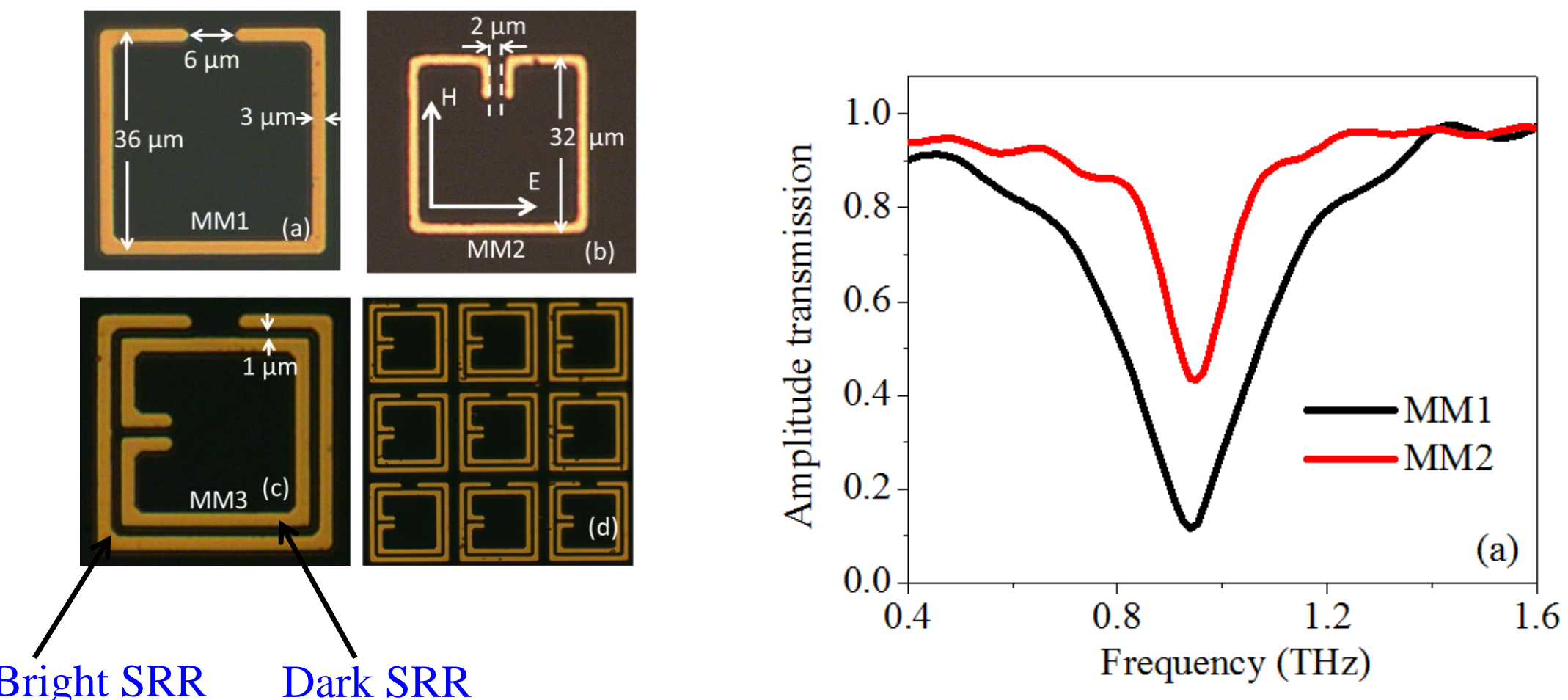

D. R. Chowdhury et al., Appl. Phys. Lett. (Accepted) 


\section{Strongly coupled planar metasurface}
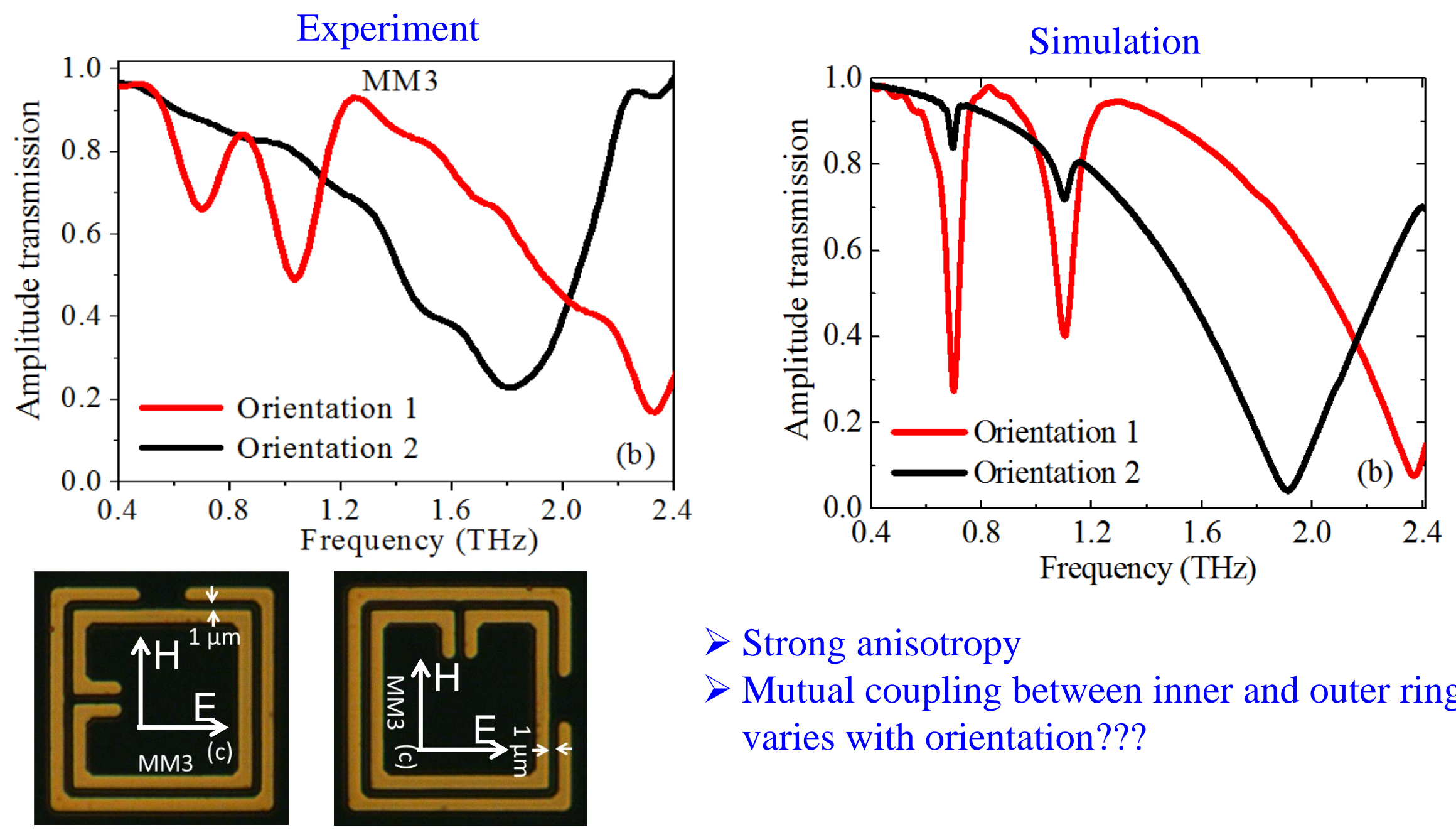

Strong anisotropy

$>$ Mutual coupling between inner and outer ring varies with orientation???

D. R. Chowdhury et al., Appl. Phys. Lett. (Accepted) 


\section{Strongly coupled planar metasurface}
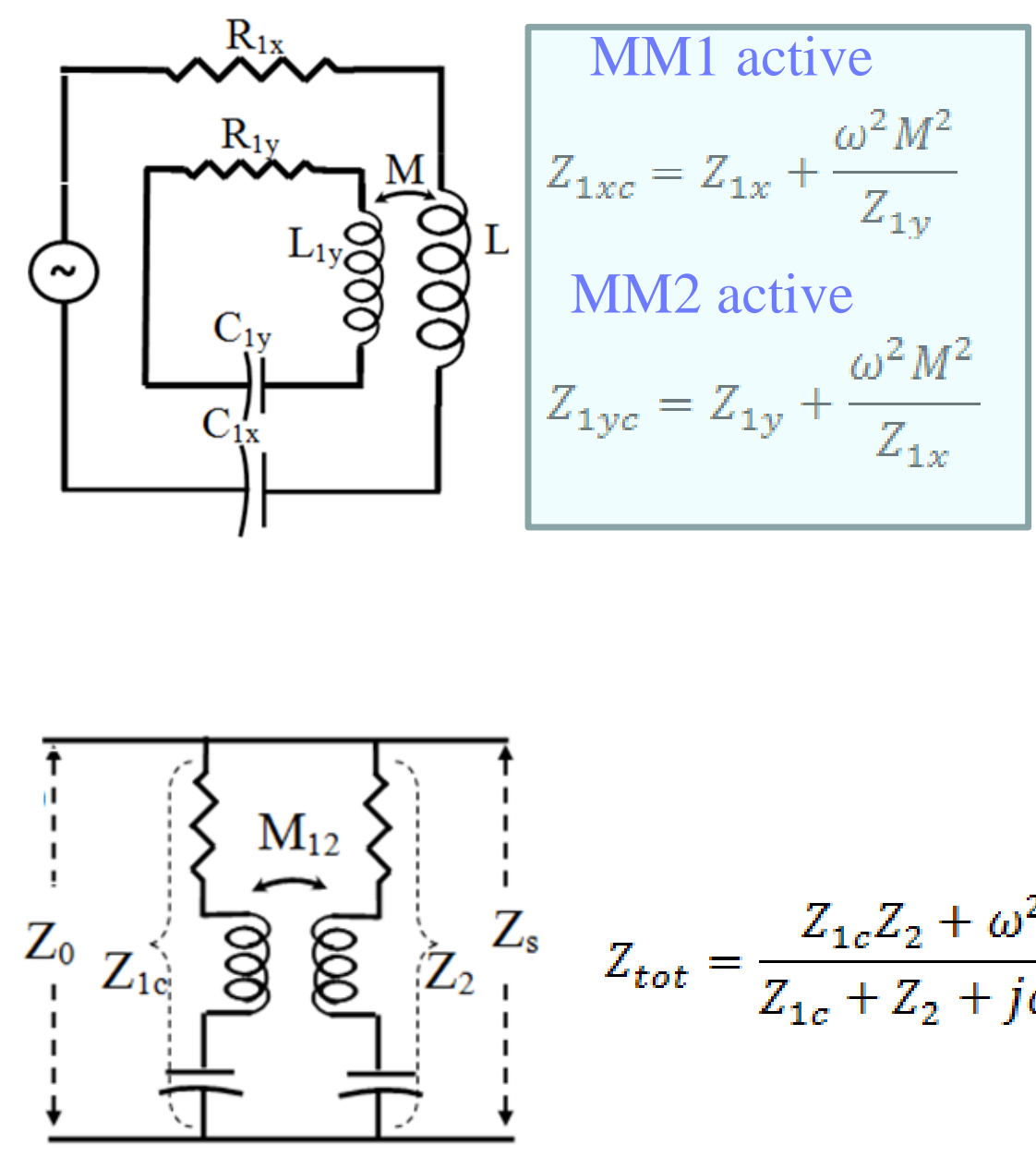

$$
Z_{\text {tot }}=\frac{Z_{1 c} Z_{2}+\omega^{2} M_{12}}{Z_{1 c}+Z_{2}+j \omega 2 M_{12}}
$$

D. R. Chowdhury et al., Appl. Phys. Lett. (Accepted)

$>$ Coupling between LC resonances of MM1 and MM2

$>$ Stronger coupling creates larger frequency separation

Analytical

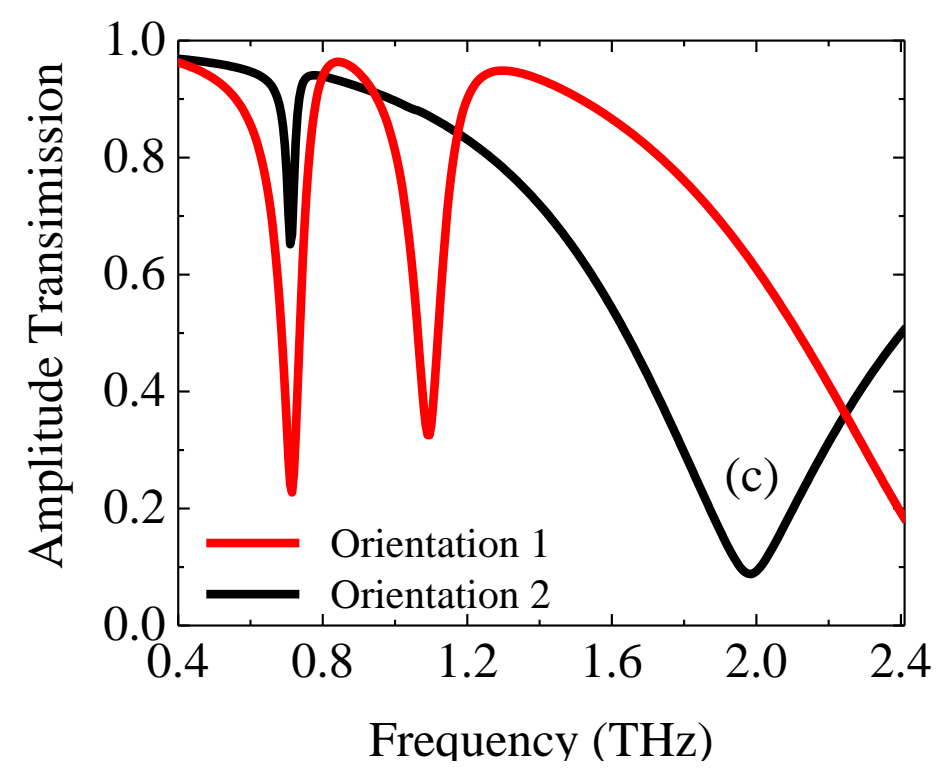

$>$ Coupling between the splitted mode and the higher order modes 


\section{An active hybrid plasmonic metamaterial}

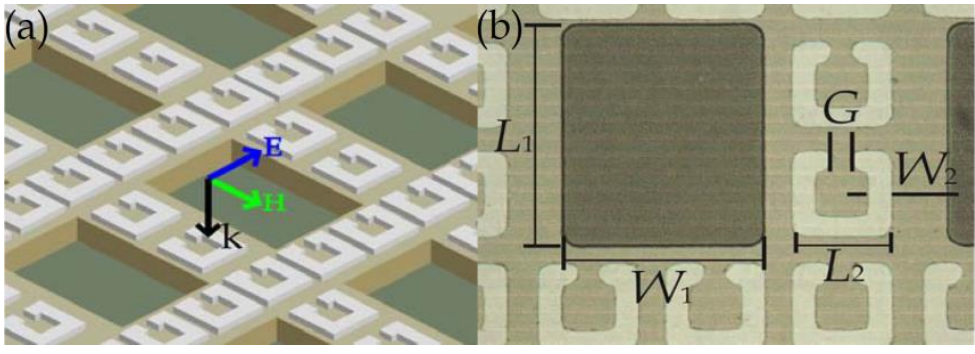

$\mathrm{L}_{1}=65 \mu \mathrm{m}, \mathrm{W}_{1}=50 \mu \mathrm{m}, \mathrm{L}_{2}=33 \mu \mathrm{m}$, $\mathrm{W}_{2}=6 \mu \mathrm{m}$, and $\mathrm{G}=5 \mu \mathrm{m}$

$>$ Substrate: Silicon on Sapphire

$>$ SRR: Aluminum (200 nm)

$>$ Silicon film thickness: $500 \mathrm{~nm}$

$>$ Plasmonic hole array: Etching silicon

J. Gu, et. al, OPTICAL MATERIALS EXPRESS 2, 1617 (2012)

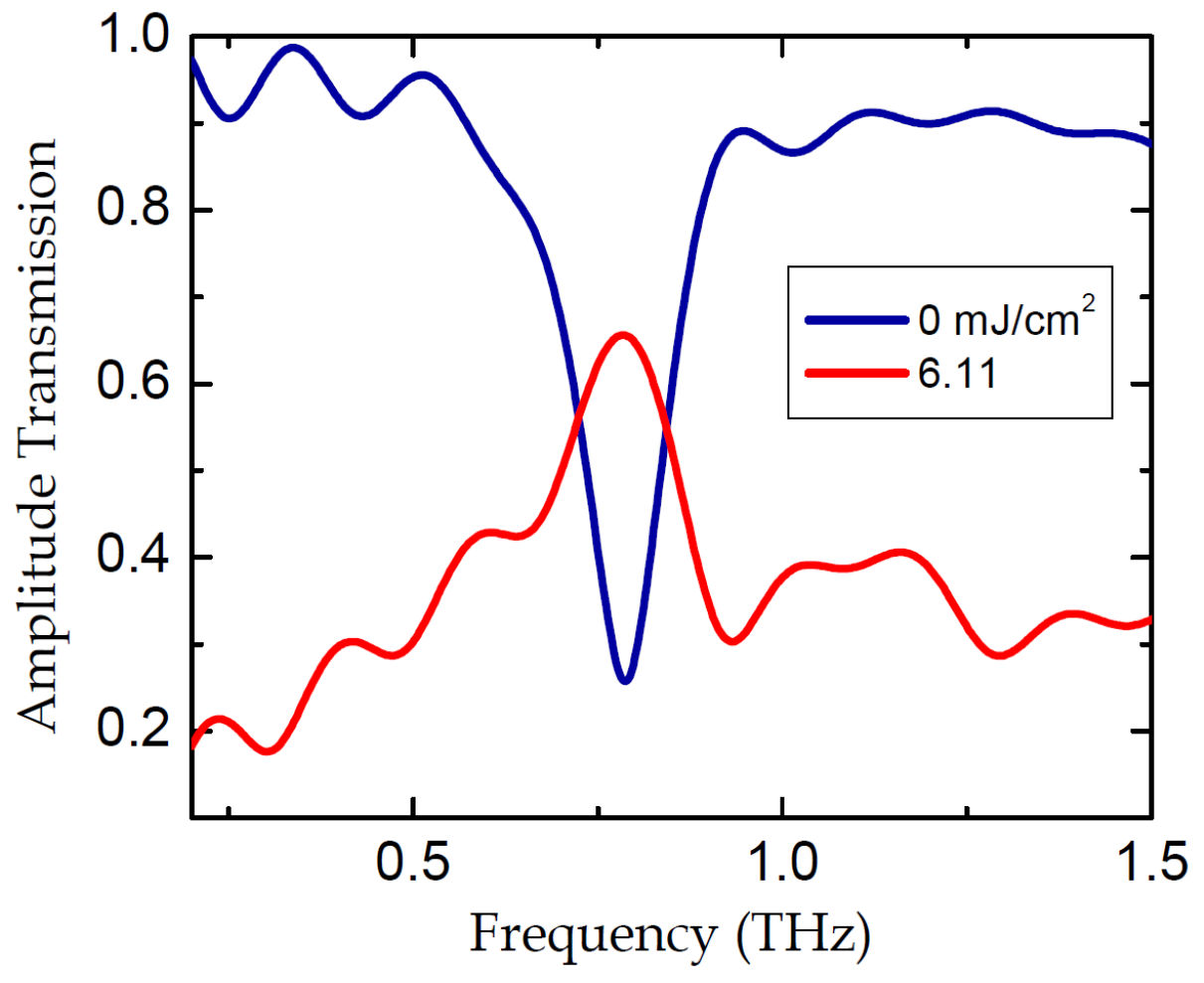

$>$ Without optical pump: SRR resonance - band-stop nature

$>$ With optical pump: Silicon becomes metallic

$>$ Behaves like a metallic hole array

$>$ Excites surface plasma polaritons, band-pass resonance 


\section{An active hybrid plasmonic metamaterial}
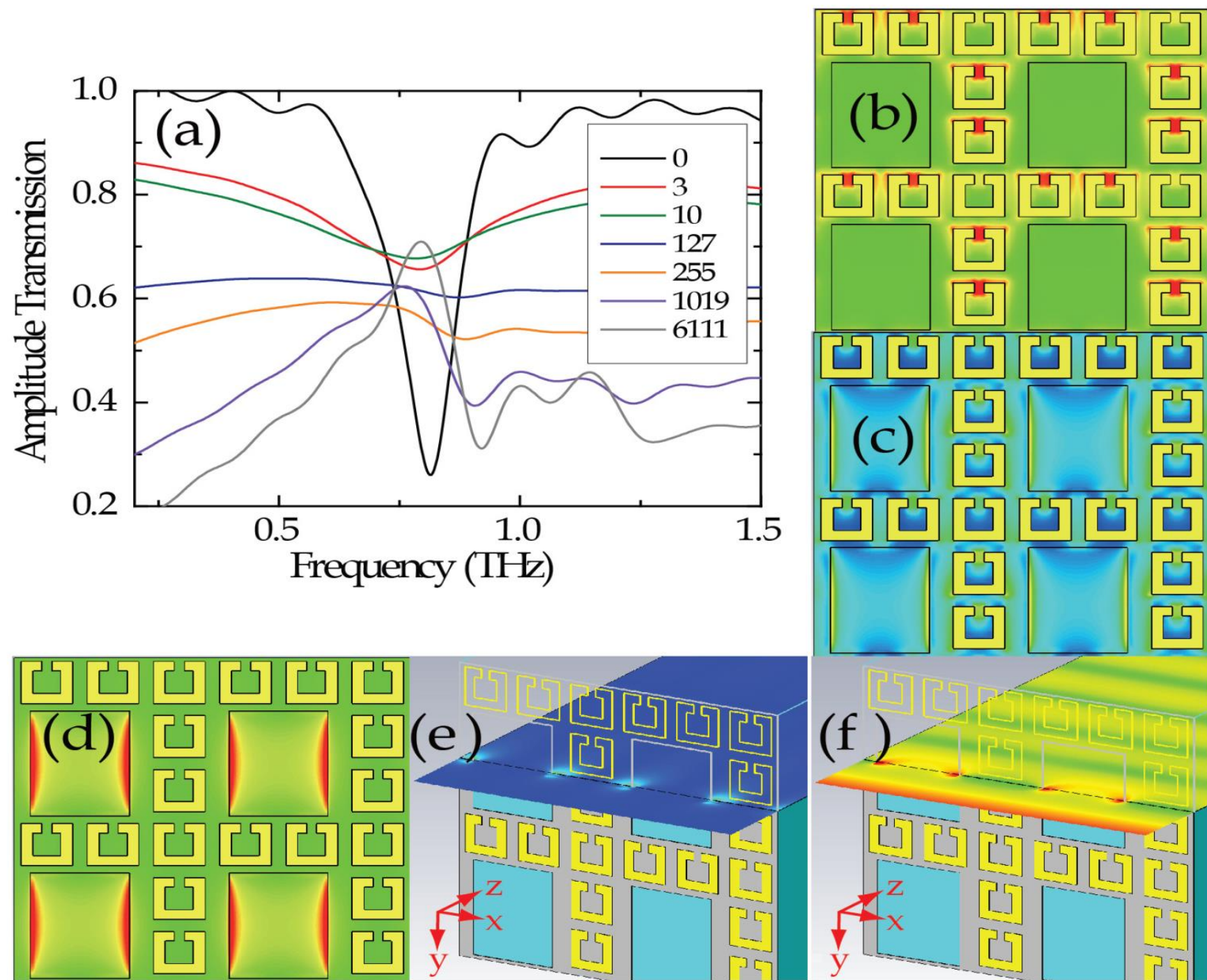


\section{Metamaterial Electro-Optical Modulator}

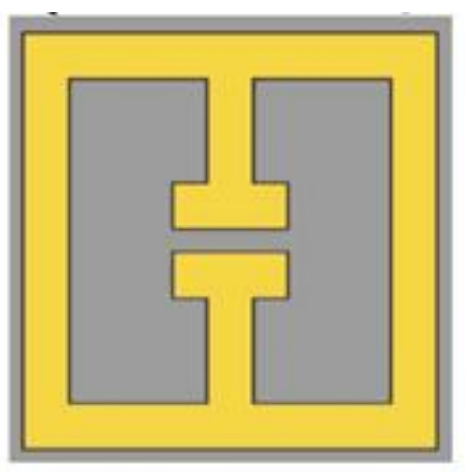

Low

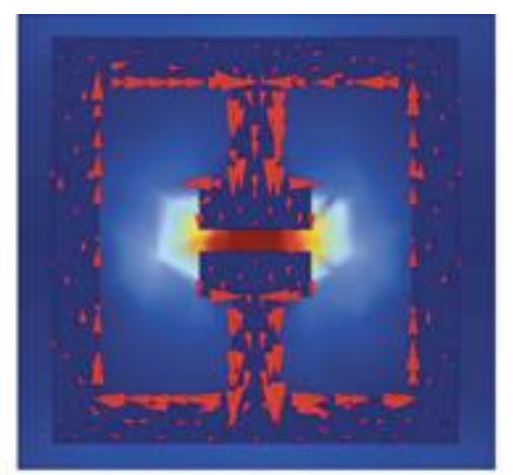

High

$>\quad$ SRR can be excited by E \& H fields

$>$ At resonance field in the gap is orders of magnitude higher than excitation field

$>\quad$ Ideal for EO modulator

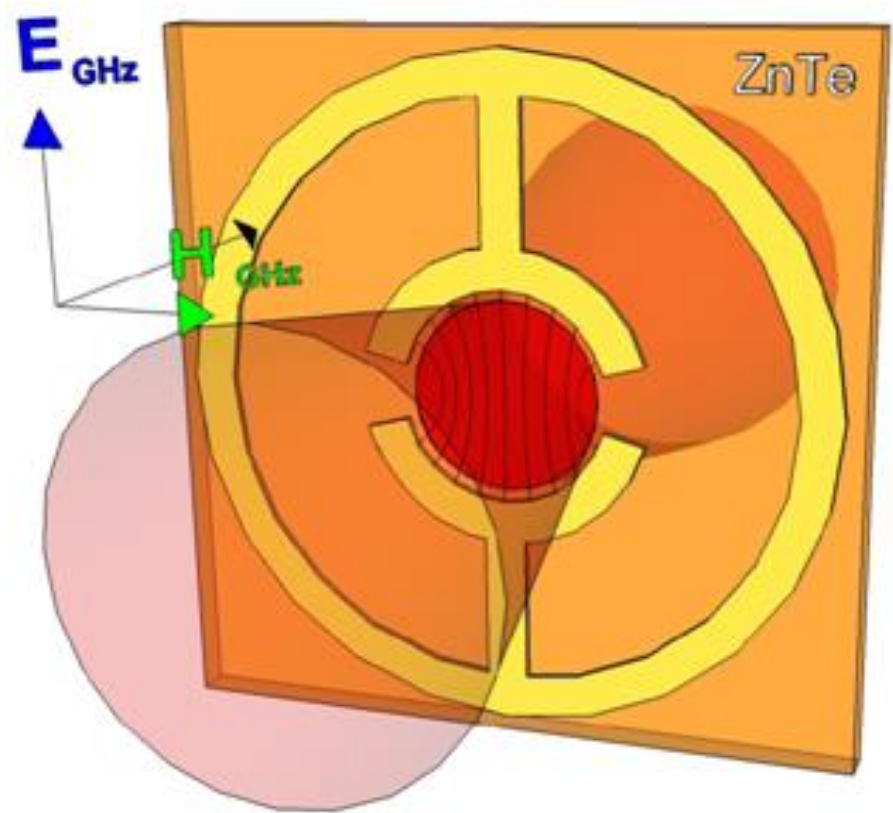

$>\quad$ Schematic of modulating IR light by microwave electric field

$>\quad$ SRR is designed to resonate at $\mathrm{GHz}$ frequencies

$>$ Fabricated on an electro-optic medium

D. Shchegolkov, et. al. Submitted to Appl. Phys. Lett 


\section{Experimental setup (EO modulation)}
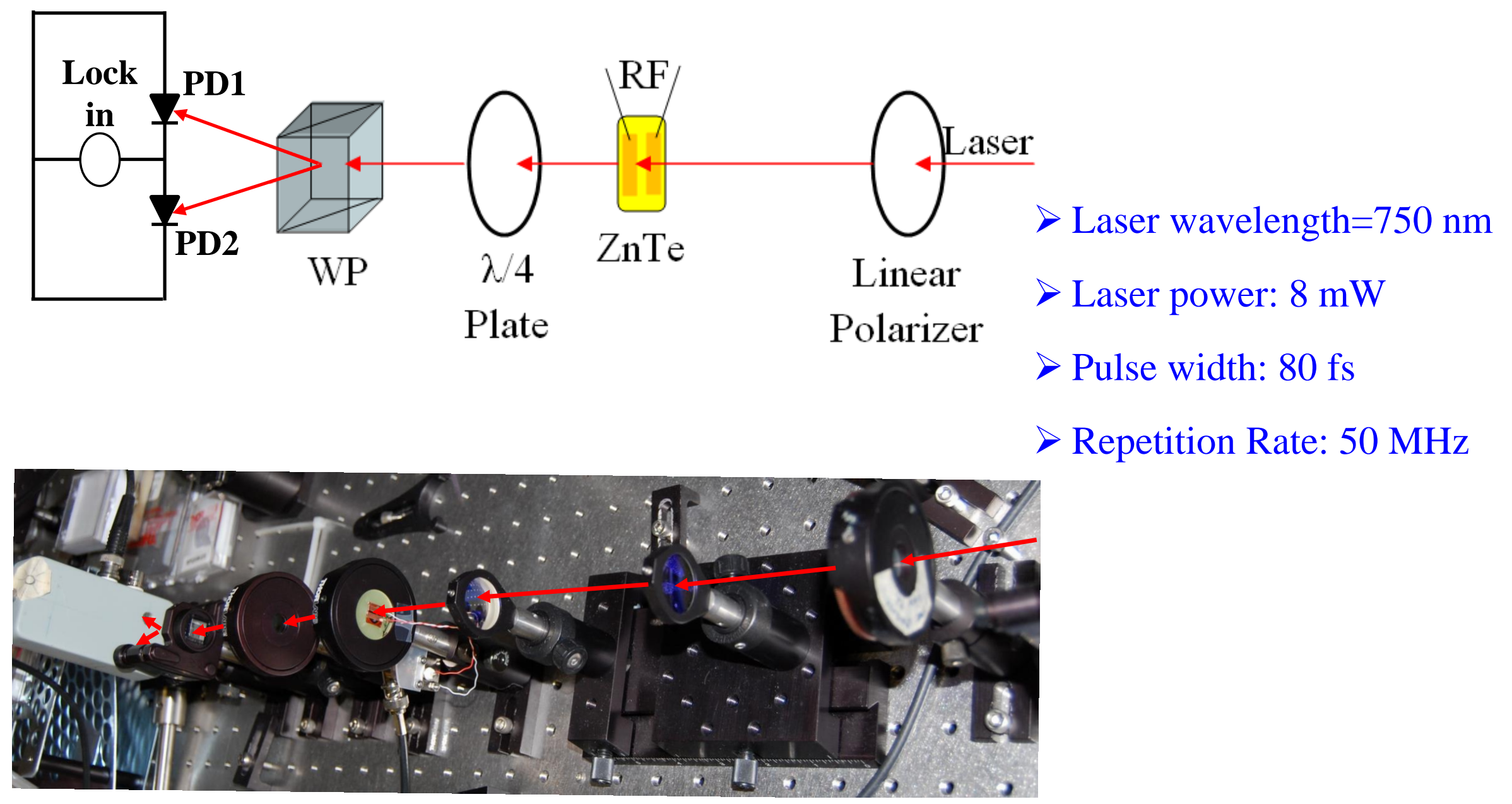


\section{SRR Fabrication}

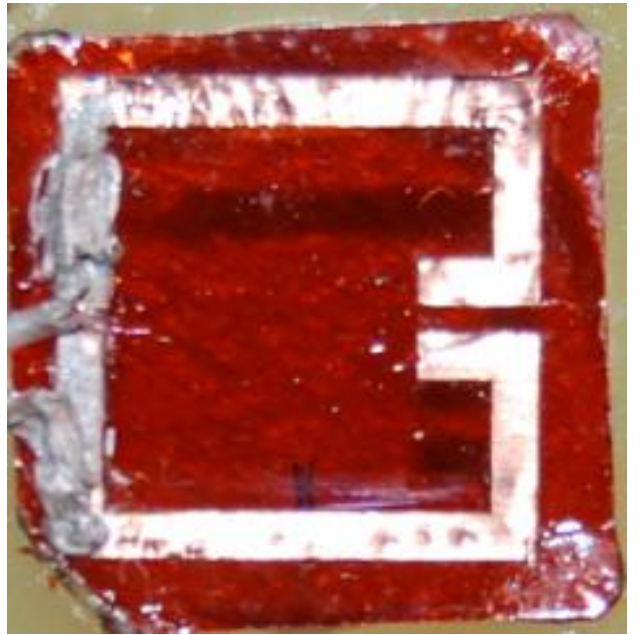

$>$ Skin depth of copper at $3 \mathrm{GHz}$ is $\approx 2 \mu \mathrm{m}$

$>$ Used short pulse laser machining

$>$ Laminate whole crystal with adhesive copper tape

$>$ Etch copper
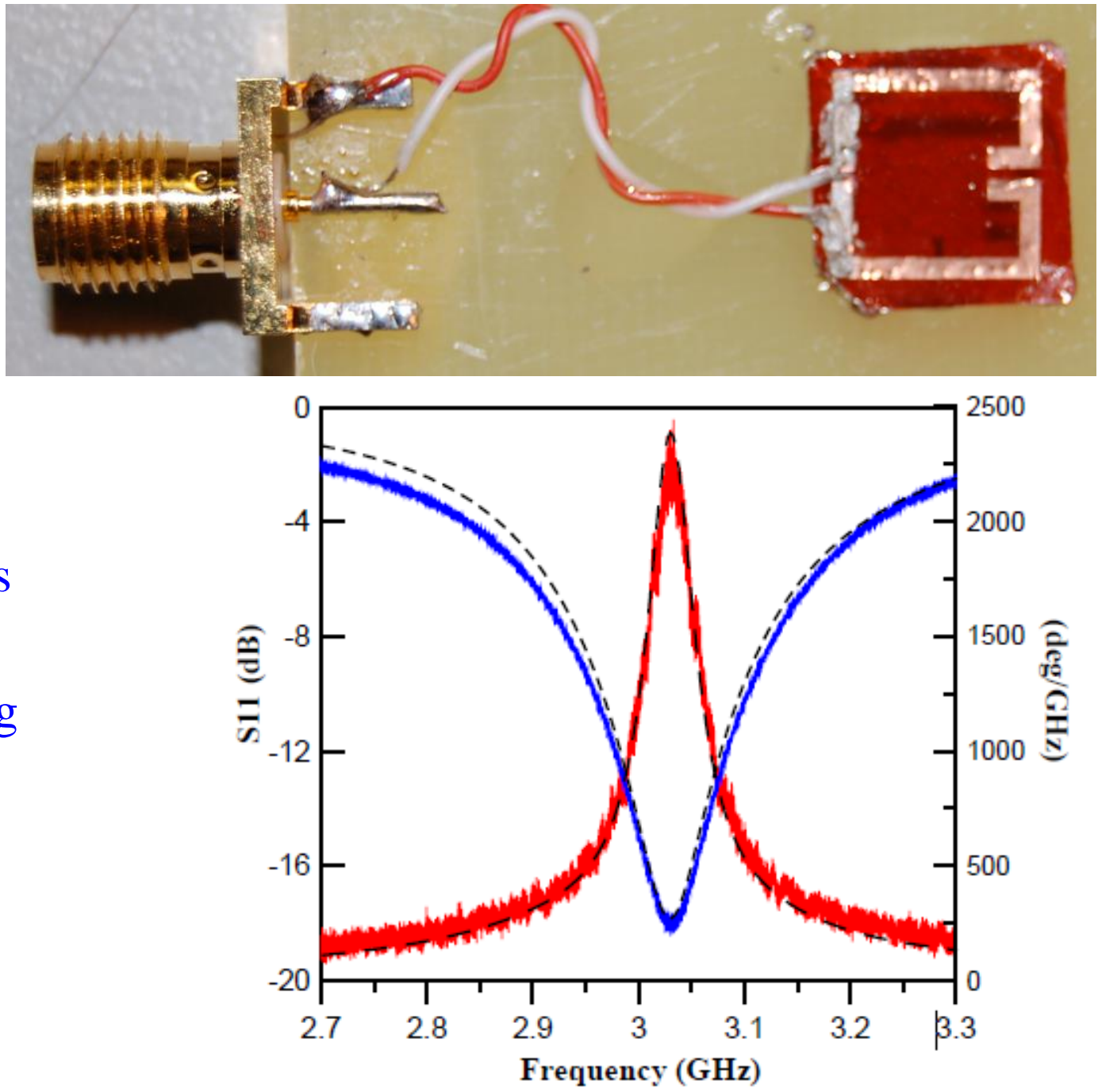


\section{EO Measurement}
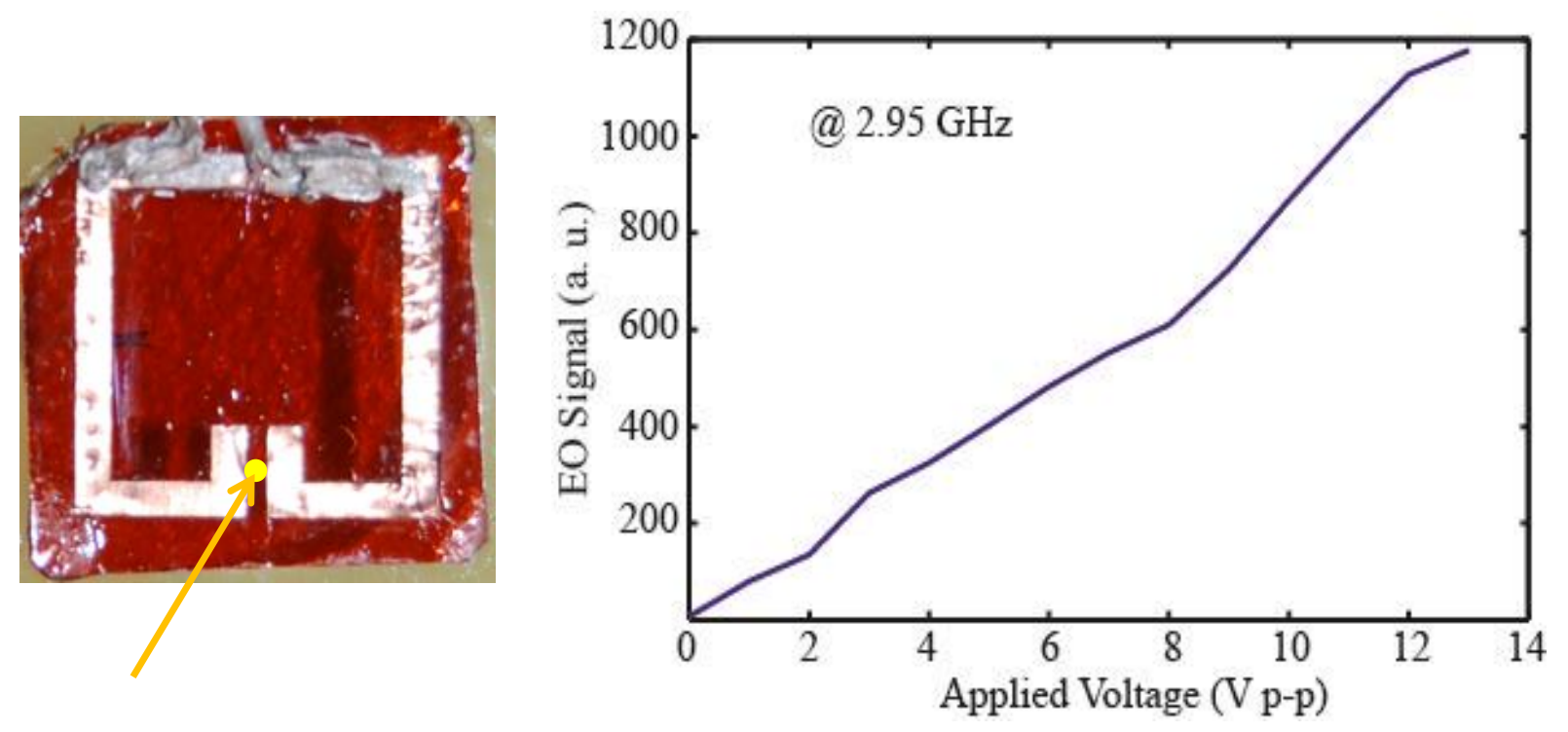

$>$ Frequency of microwave is fixed @ 2.95 $\mathrm{GHz}$

$>$ Probe at the middle of the resonant gap

$>$ Optical beam diameter on the sample is $\sim 100 \mu \mathrm{m}$

$>$ EO signal increases linearly with applied voltage 


\section{Frequency dependence of EO signal}
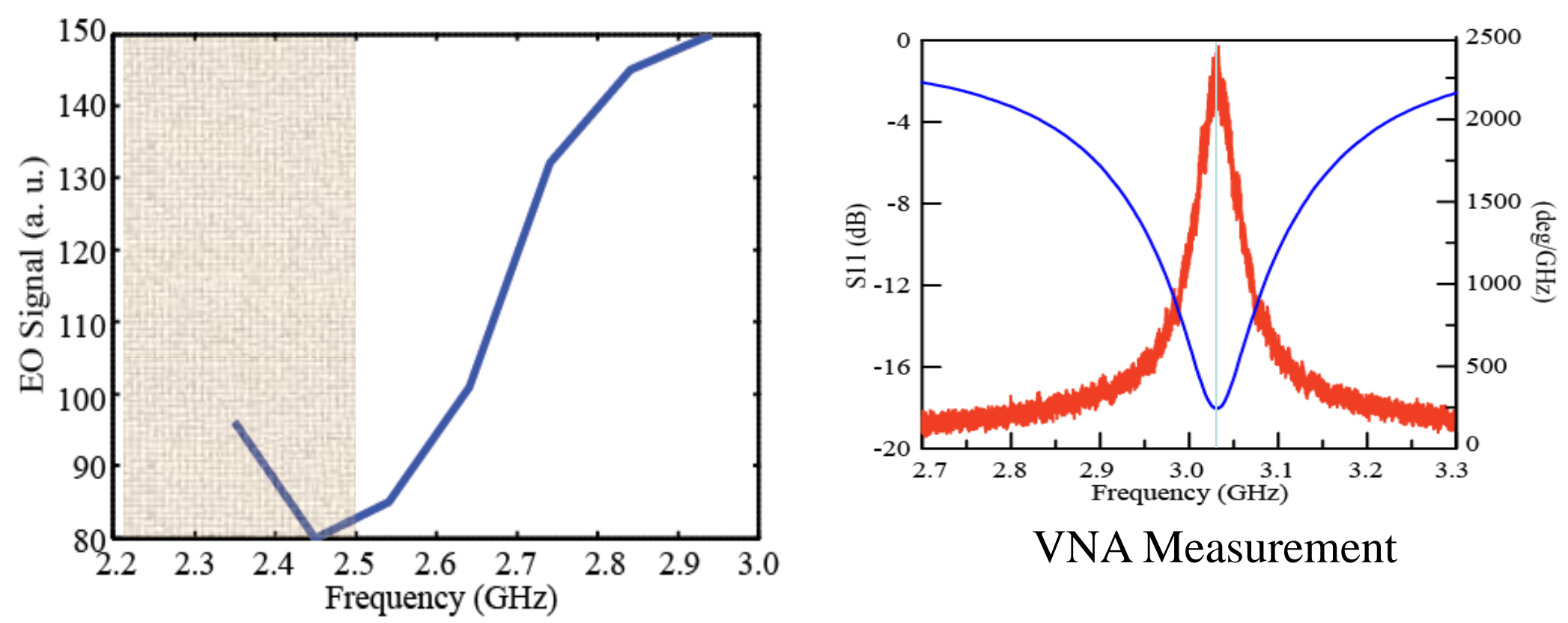

VNA Measurement

$>$ Keep the applied voltage p-p amplitude the same

$>$ 2.5-3.0 GHz is the specified PC board operational frequency range

$>$ EO signal rolls-off as the microwave frequency offsets from the resonance of the SRR

$>$ Qualitatively follows simulated voltage in the gap 


\section{Spatial distribution of EO signal: experiment}

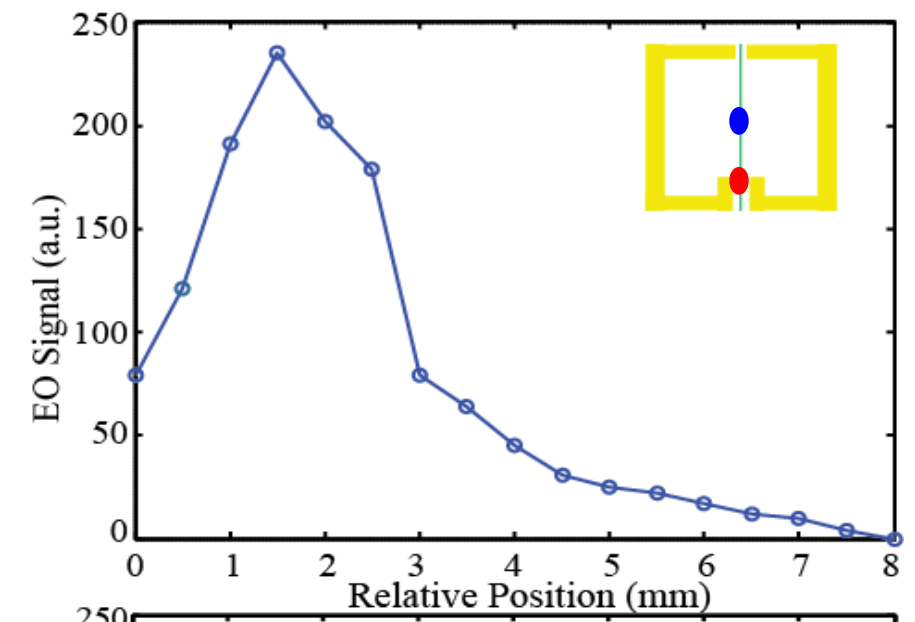

$>$ EO signal in the SRR gap is five times higher compared to signal at the SRR center

$>$ Horizontal scanning shows the presence of orthogonally polarized electric filed component on both

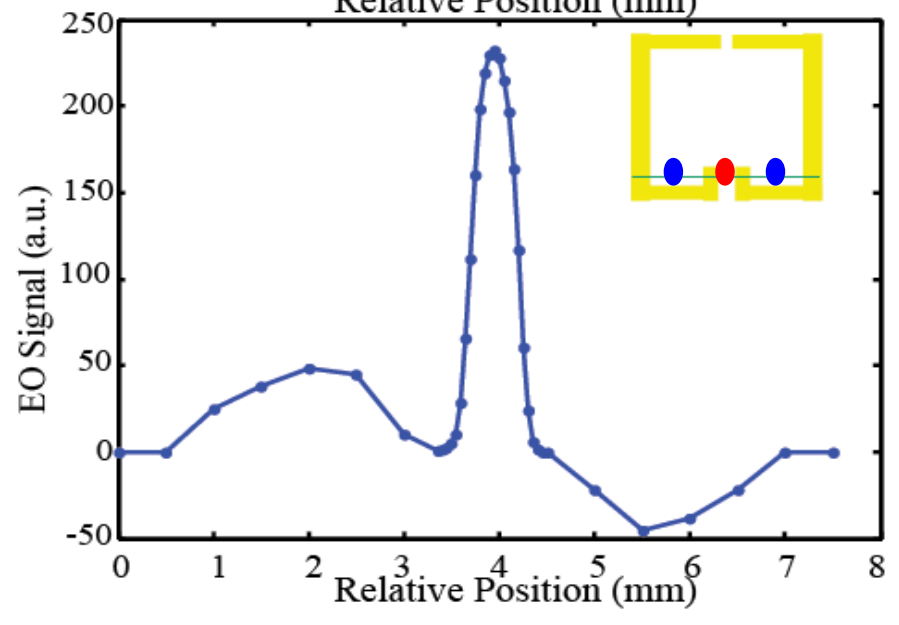
sides of the gap

$>$ Field enhancement is the highest inside the gap

$$
\mathrm{Q} \approx 7
$$




\section{D Field distribution}

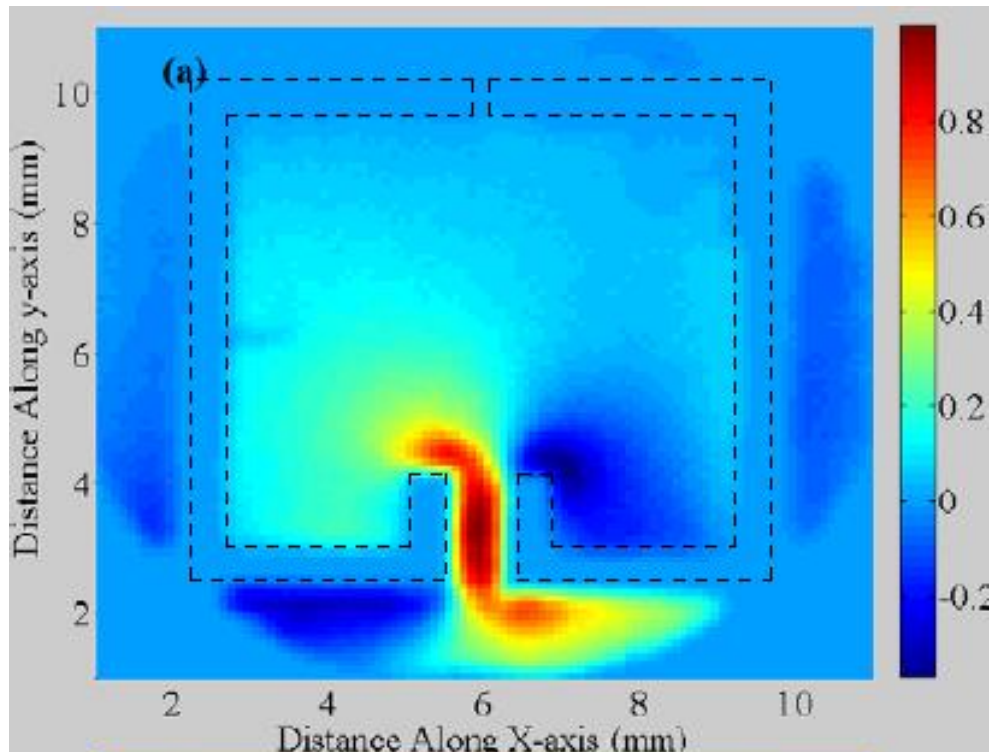

(b)

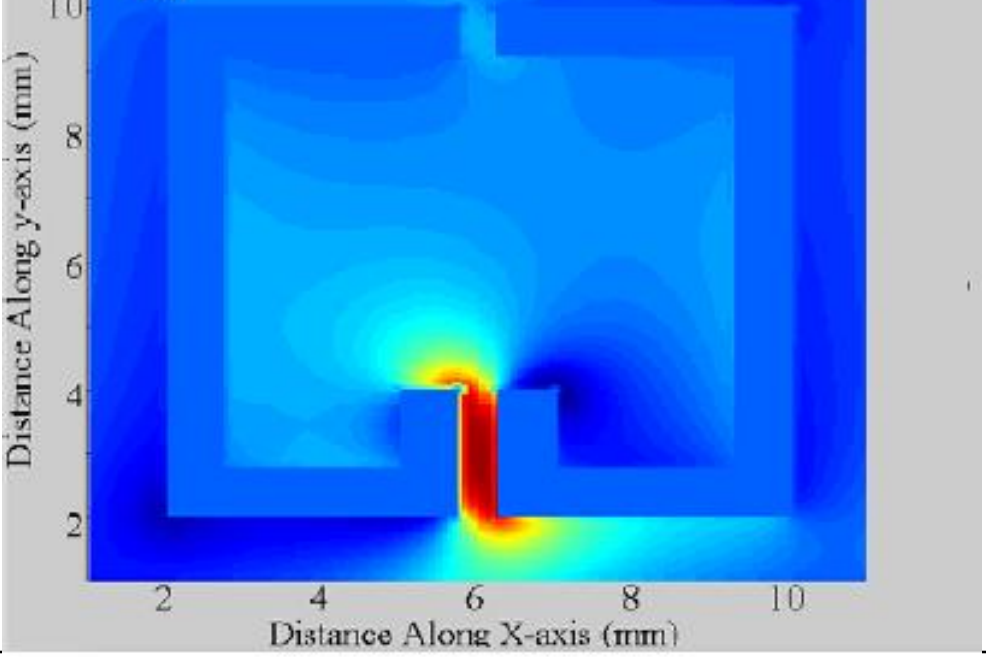

Center for Integrated Nanotechnologies

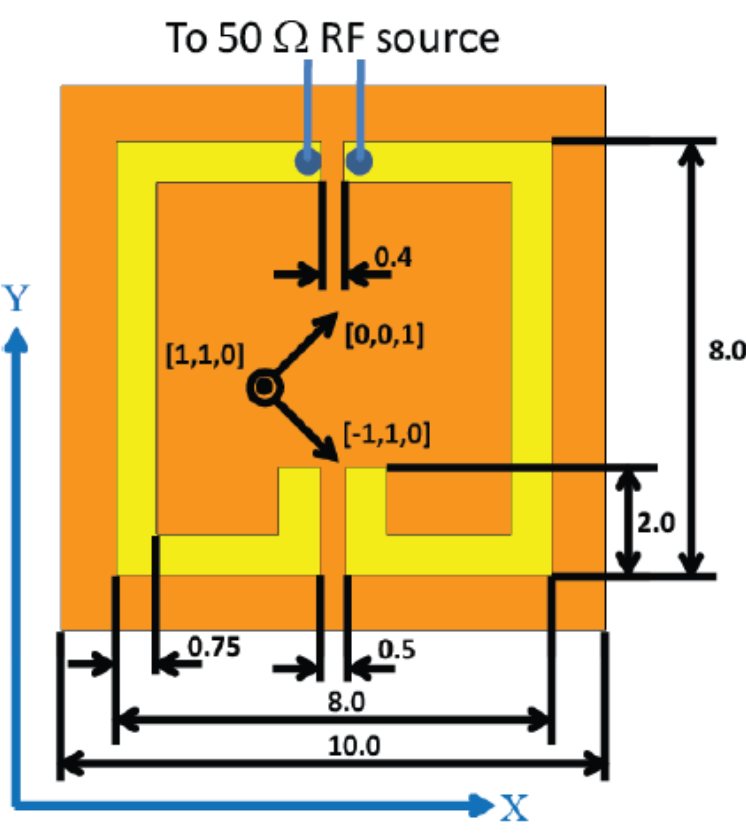

$$
\begin{aligned}
& \Gamma=\frac{\pi d}{\lambda_{0}} n_{0}^{3} r_{41} E_{r f} \sqrt{1+3 \cos ^{2} \alpha} \\
& I=\rho \frac{\pi d}{\lambda_{0}} n_{0}^{3} r_{41} E_{r f}\left|E_{0}\right|^{2} \sin \alpha
\end{aligned}
$$




\section{Summary}

$\square$ Engineered Metamaterials at $\mathrm{THz}$ frequencies, Strong resonance response

$\square$ Understanding basic properties with respect to design parameters

$\square$ Investigated the coupling mechanism and the corresponding effect on resonances

$\square$ We are able to actively modulate $\mathrm{THz}$ radiation using optical and thermal controls

$\square$ Demonstrated metamaterial based EO modulator 


\section{Team and Collaborators}

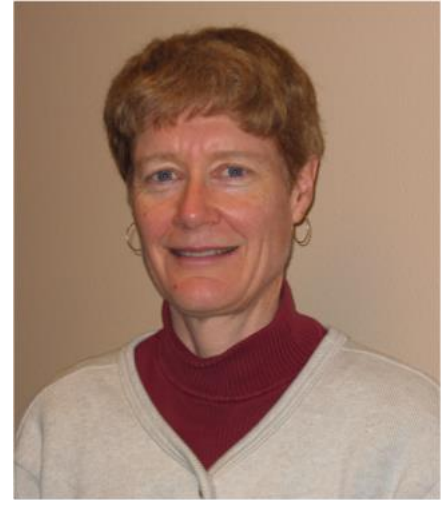

Toni Taylor

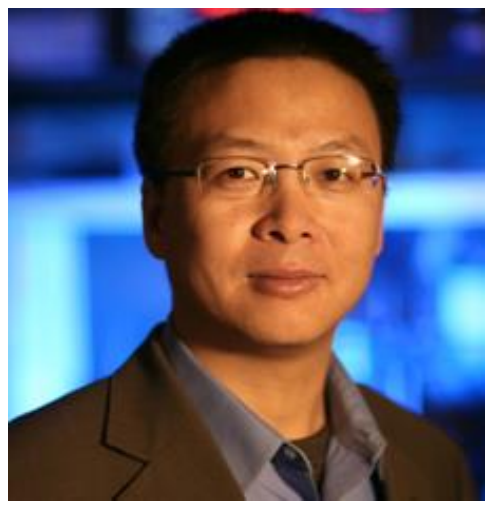

Hou-Tong Chen

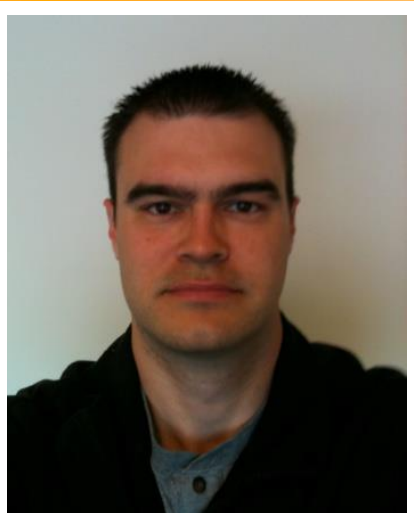

John O’Hara

\section{Matthew Reiten Dibakar Chowdhury Ranjan Singh Jiangfeng Zhou}

LANL: Quanxi Jia, Stuart Trugman, Diego Dalvit, Lawrence Earley, Michael Holloway, Evgenya Smirnova, Dmitry Shchegolkov

Oklahoma State University: Weili Zhang

Boston University: Richard Averitt

Boston College: Willie Padilla

University of California, Santa Barbara: Arthur Gossard

Sandia National Laboratory: Igal Brener, Mark Lee, Michael Cich

Duke University: David Smith, Nan Jokerst 


\section{Center for Integrated Nanotechnologies}

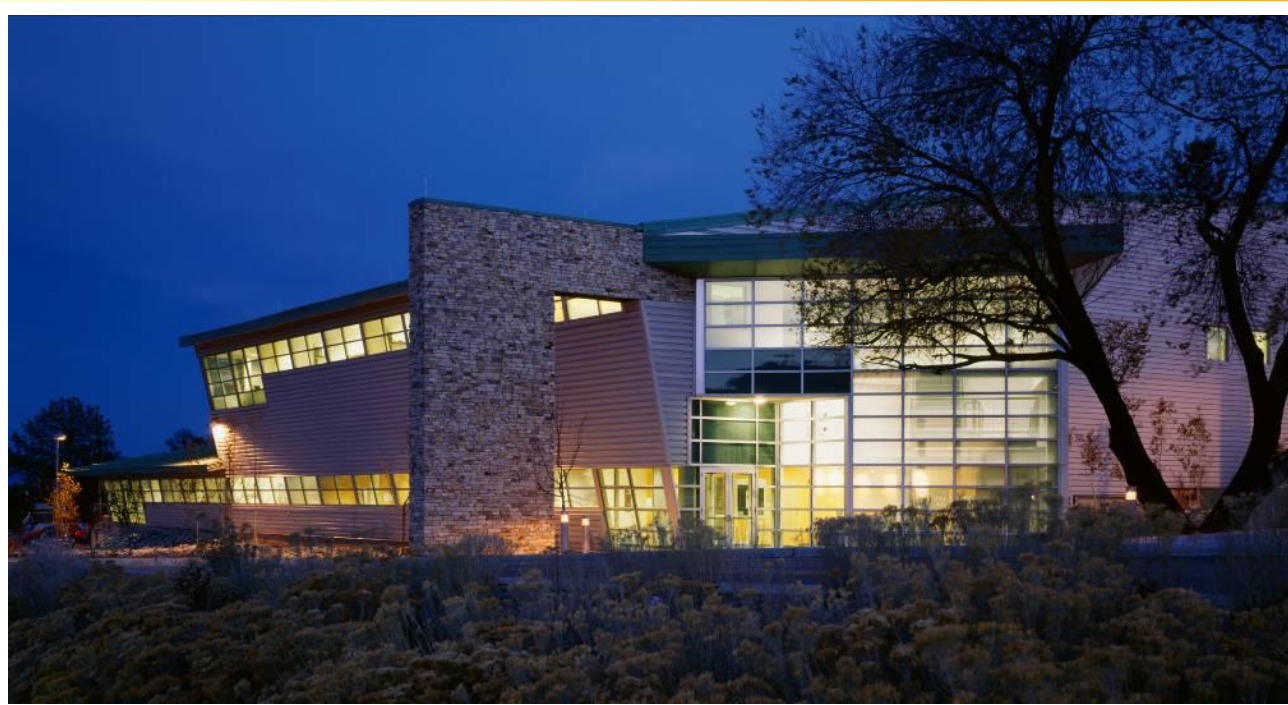

"One scientific community focused on nanoscience integration"

Los Alamos National Laboratory

State-of-the-art facilities

$>$ Goal: Develop innovative approaches for nano-scale integration

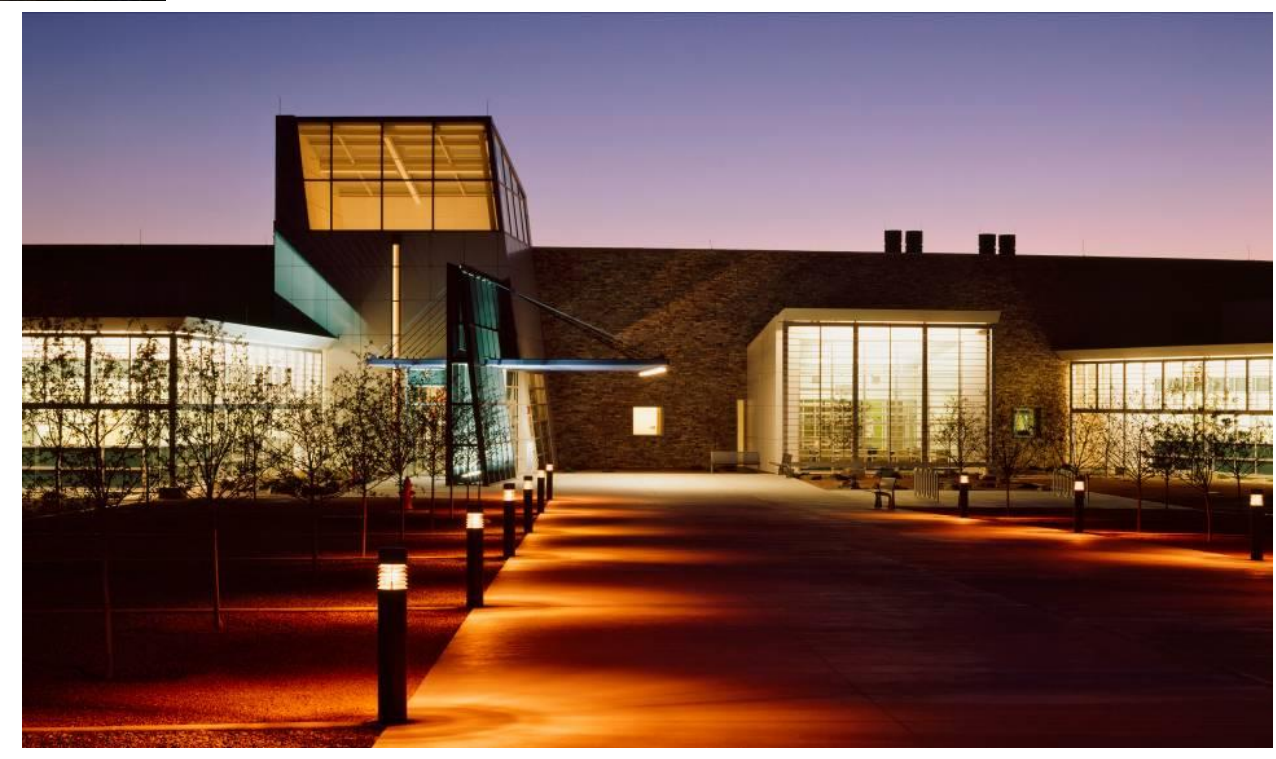

http://CINT.lanl.gov

Sandia National Laboratory 


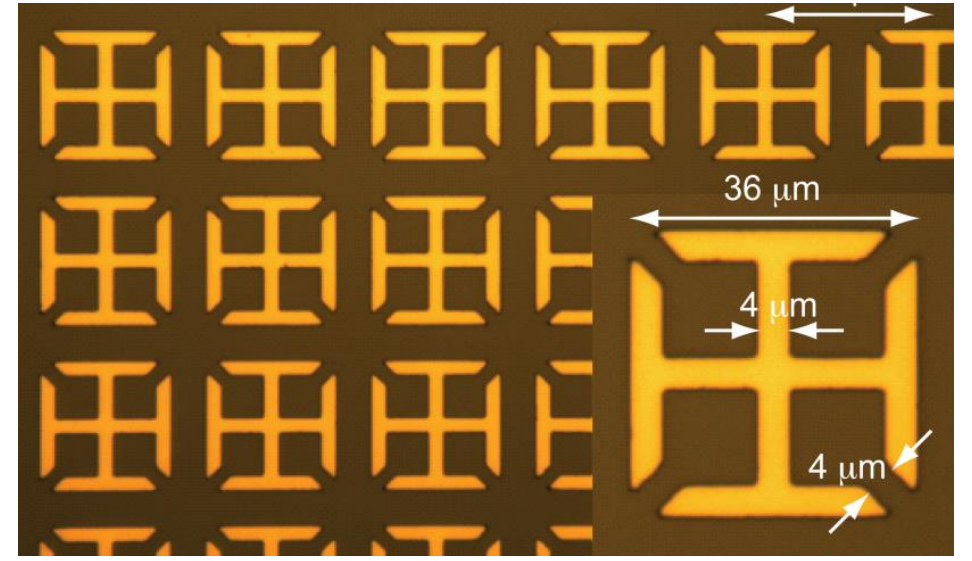

$\omega_{0}=1 / \sqrt{L C} \sim 1 / n$

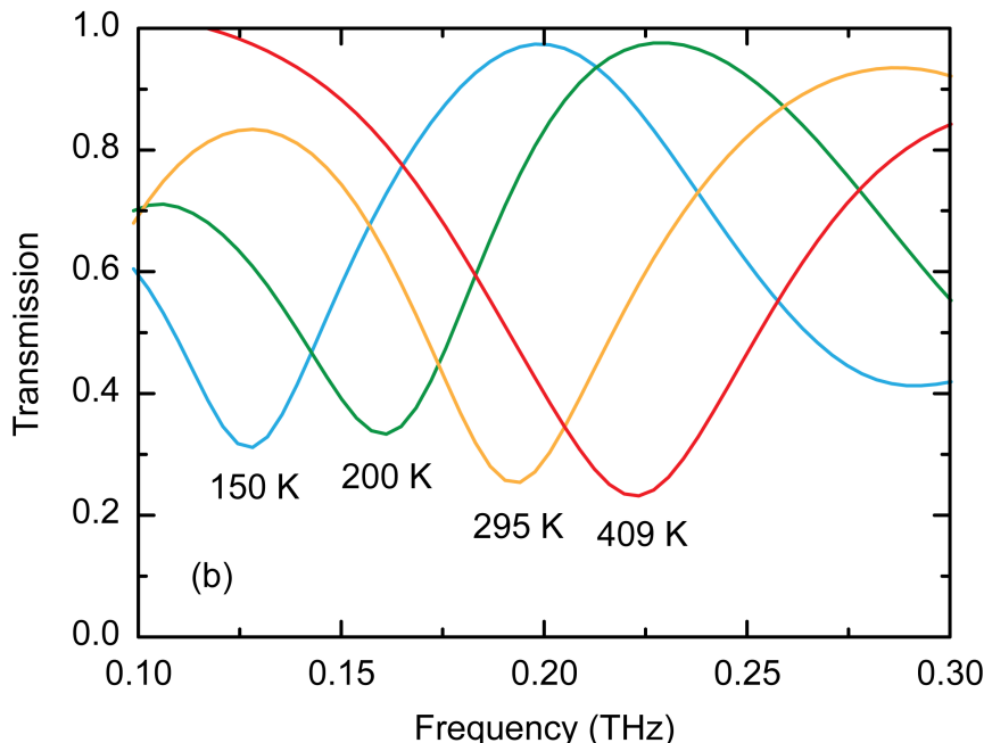

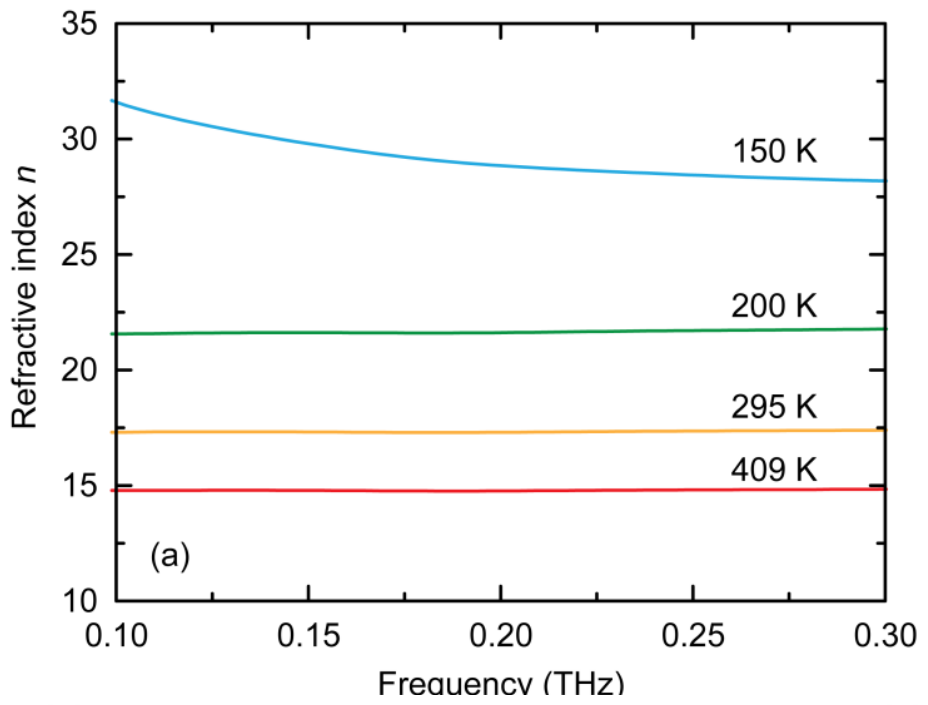

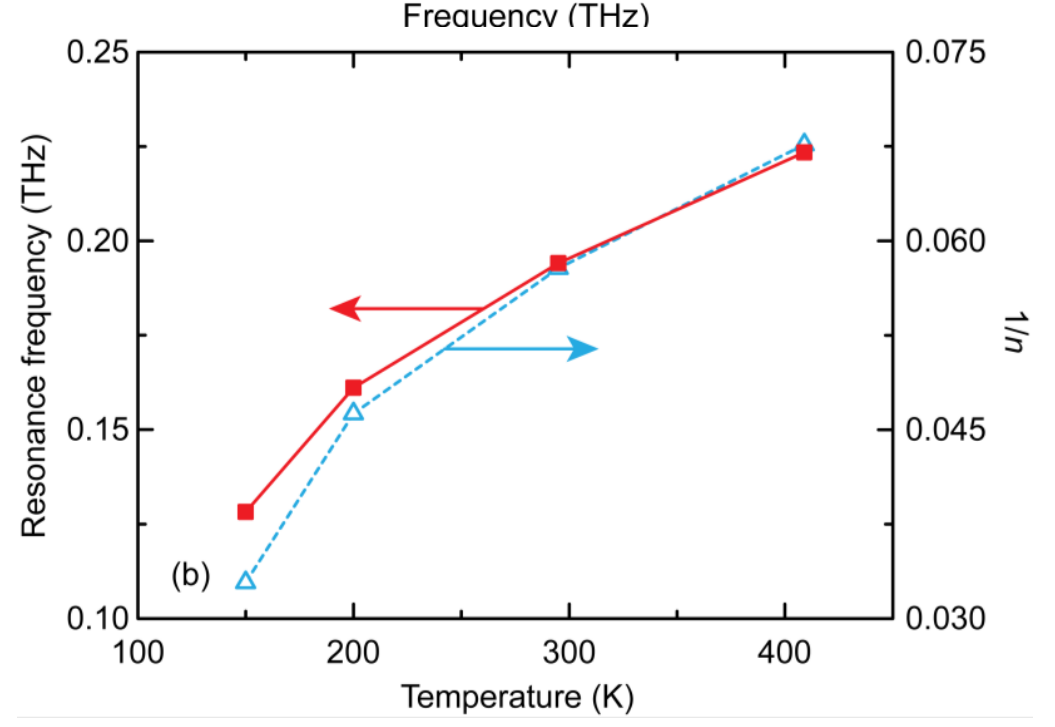

Singh et al., Opt. Lett. 36, 1230 (2011). 


\section{Superconducting THz MMs enable thermal tunability}

High quality epitaxial YBCO film on LAO by Pulsed Laser Deposition with TC $~ 90 \mathrm{~K}$ and thickness $180 \mathrm{~nm}$
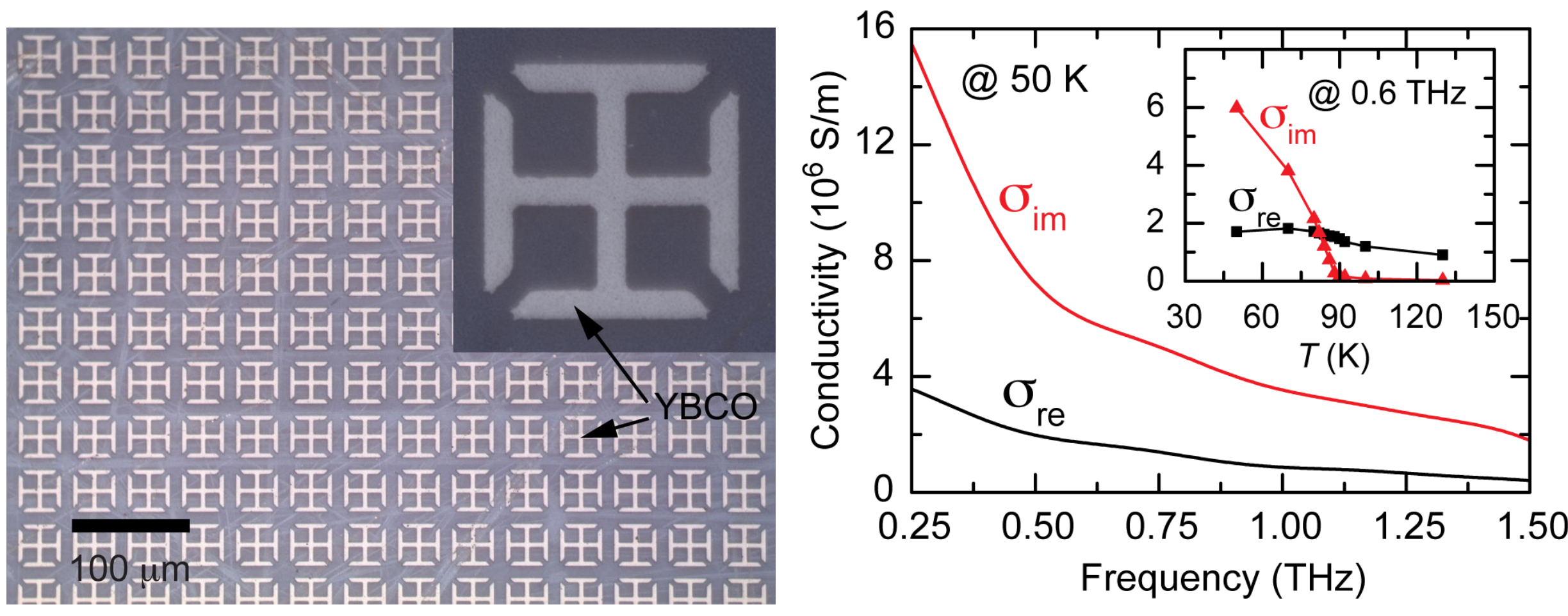

Experimentally measured complex conductivity of YBCO 


\section{Thermally Tunable Metamaterial Resonance}

$180 \mathrm{~nm}$ thick YBCO MM

$50 \mathrm{~nm}$ thick YBCO MM
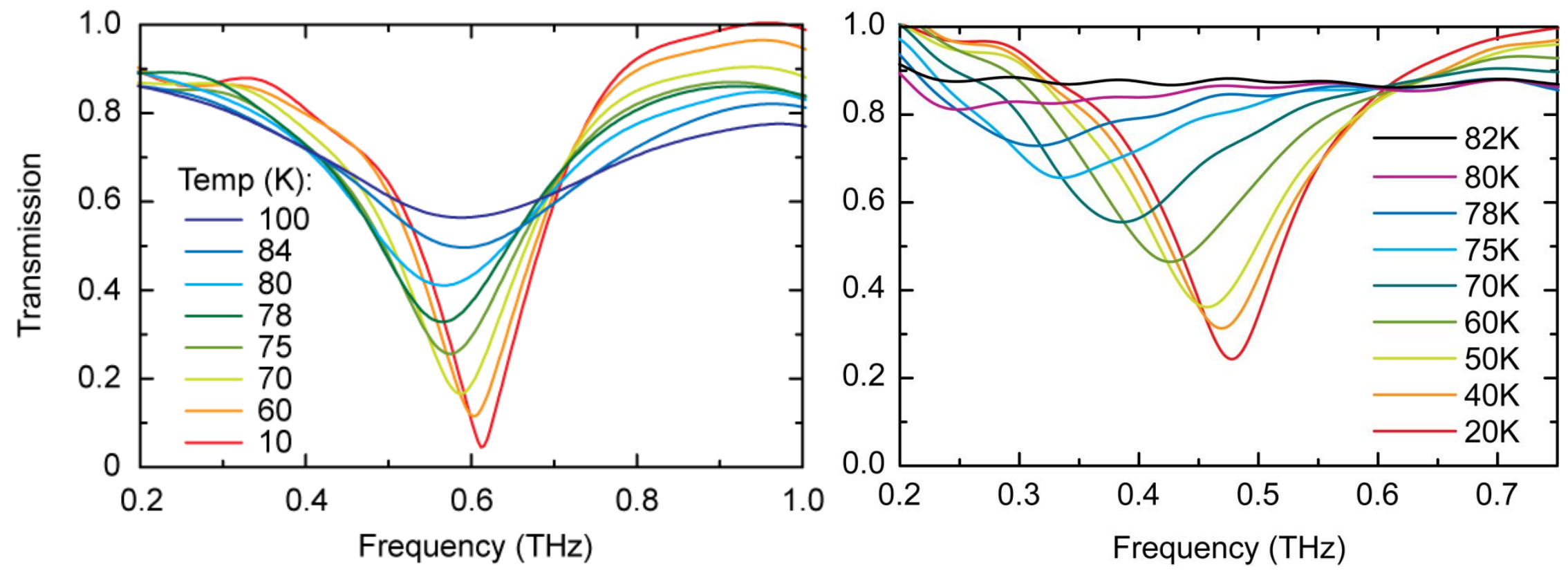

$>$ Switching resonance strength and shifting resonance frequency

$>$ Decreasing the thickness results in:

- A red-shift of the resonance frequency

- A larger tuning range and higher tuning efficiency 


\section{MM resonance tuning by photoexcitation}

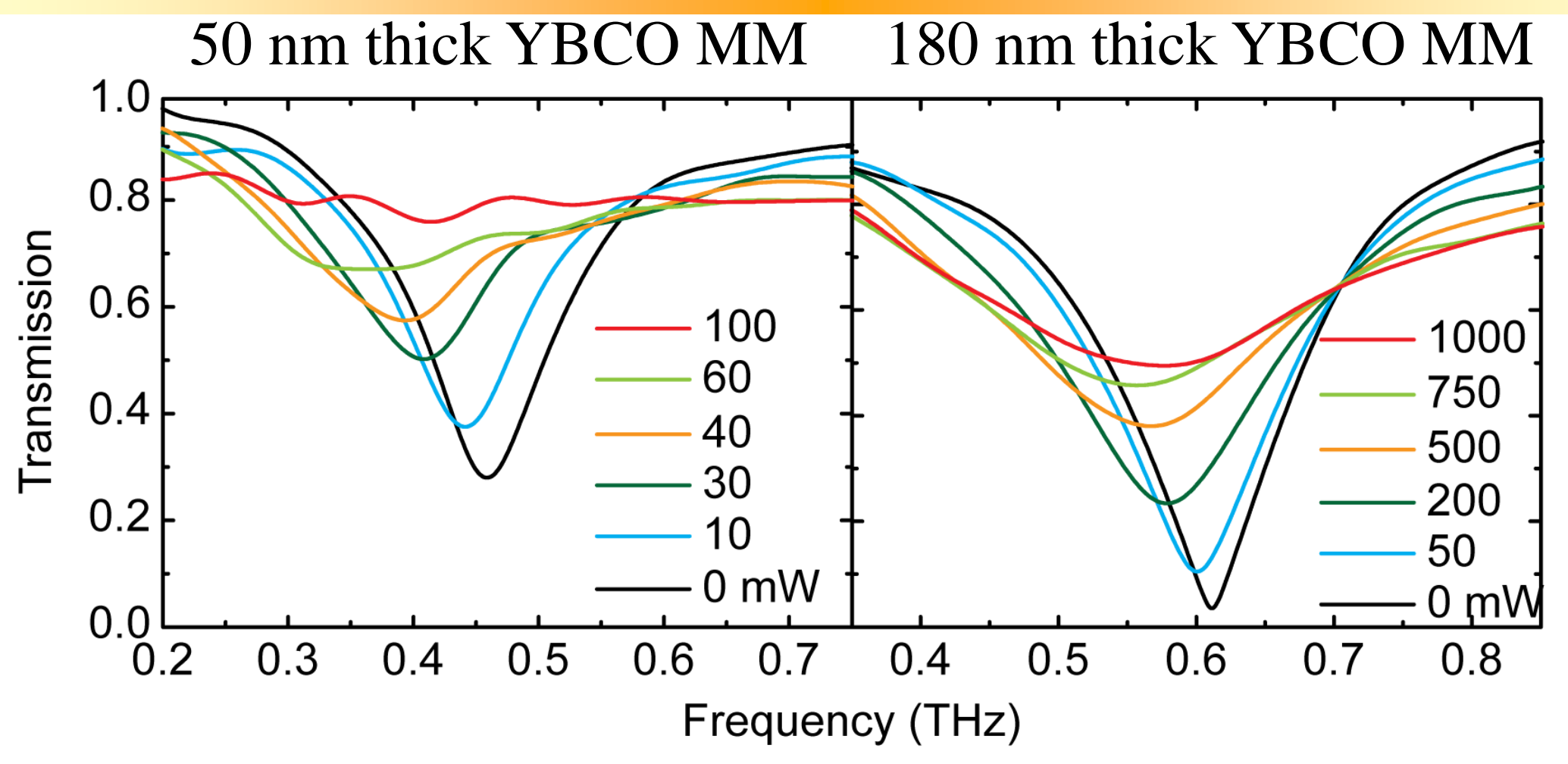

Metamaterials made from different thicknesses of YBCO films

$>$ Resonance tuning by photoexcitation depends on thickness 


\section{Ultrafast Dynamics}

Optical Pump THz Probe
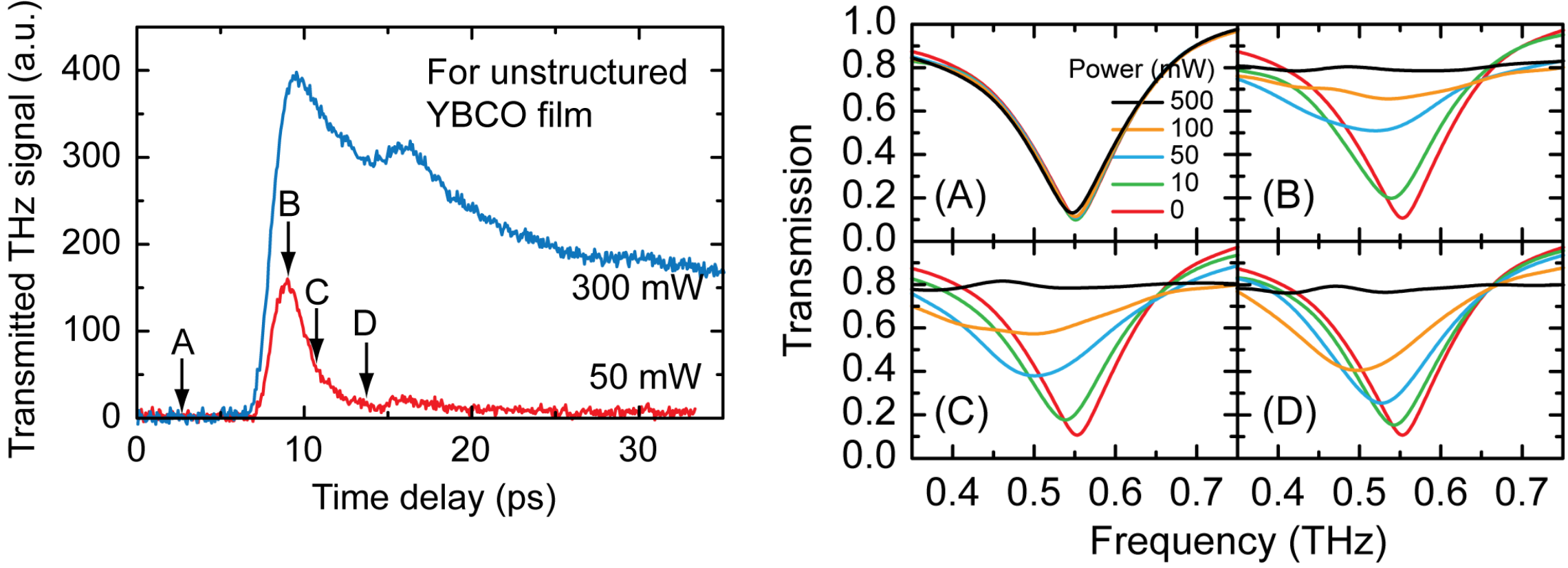

$>$ Relaxation (recombination) time is a few ps

$>$ Increasing the pump power results in a long relaxation tail due to thermal effects 


\section{Optical THz Switch: Optical-Pump Terahertz-Probe}

$\left.\begin{array}{l}1 \mathrm{KHz}, \sim 100 \mathrm{fs} \\ @ 800 \mathrm{~nm}\end{array}\right\} \quad$ Beam splitters

\section{Pump}

IE-

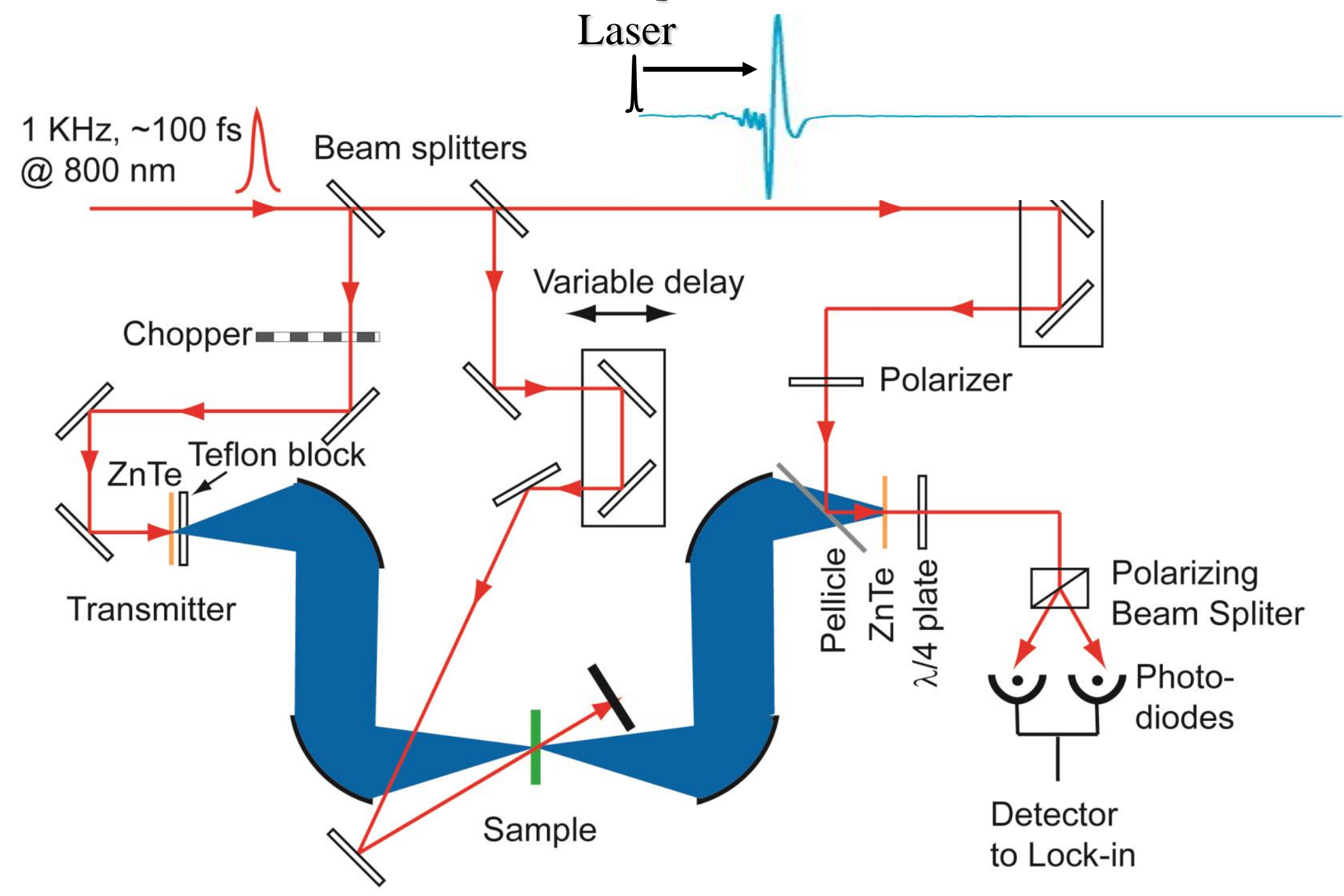




\section{Interaction Between Layers}
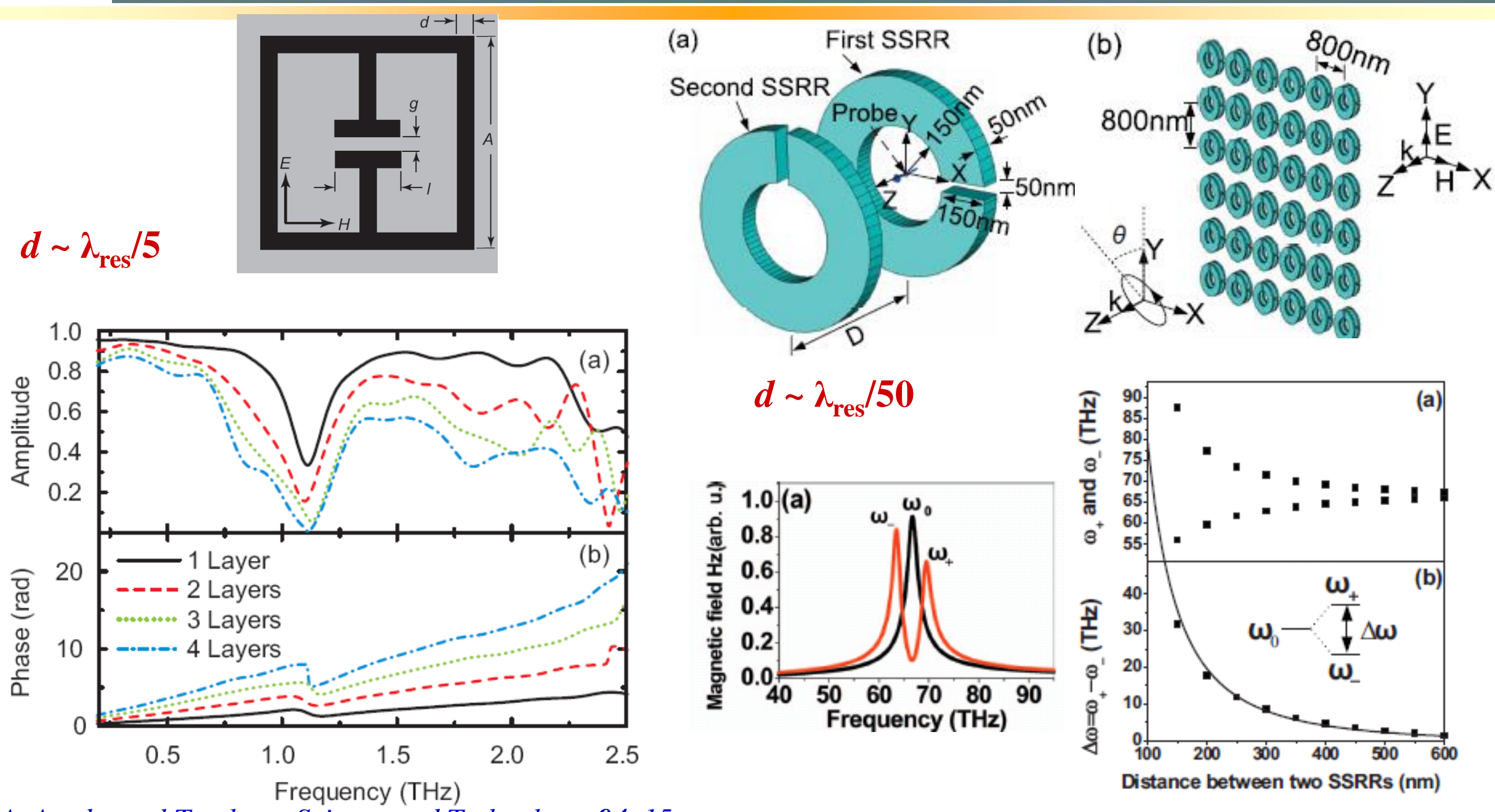

A. Azad, et. al Terahertz Science and Technology, 94, 15 (2009)

H. Liu, et. al Phys. Rev. 76, 073101 (2007) 


\section{Coupled SRRs (Bi-Layer)}
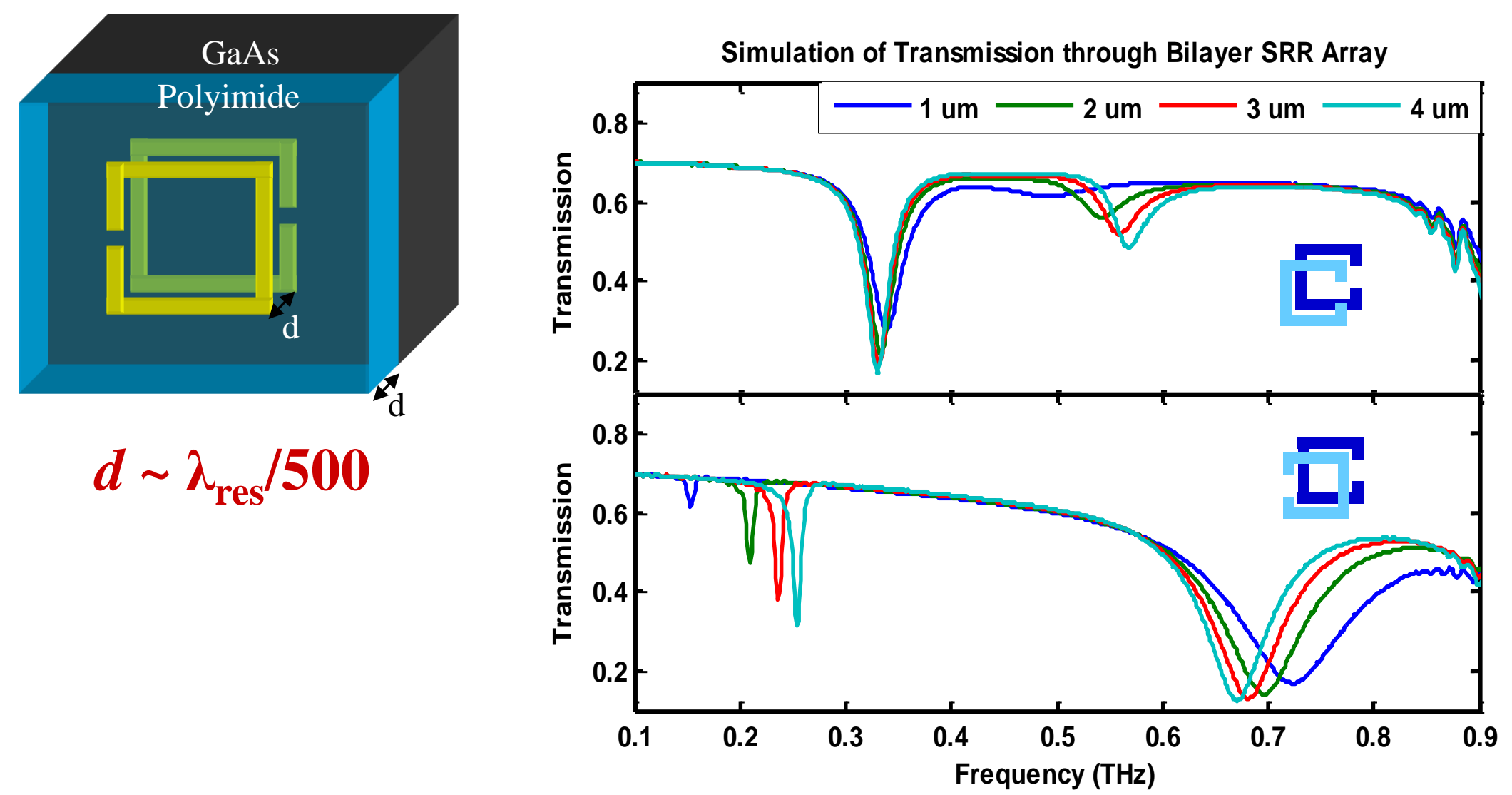

M. T. Reiten, et. al, App. Phys. Lett. 98131105 (2011) 


\section{Simulation. vs. Measurement for Anti-Aligned SRR}

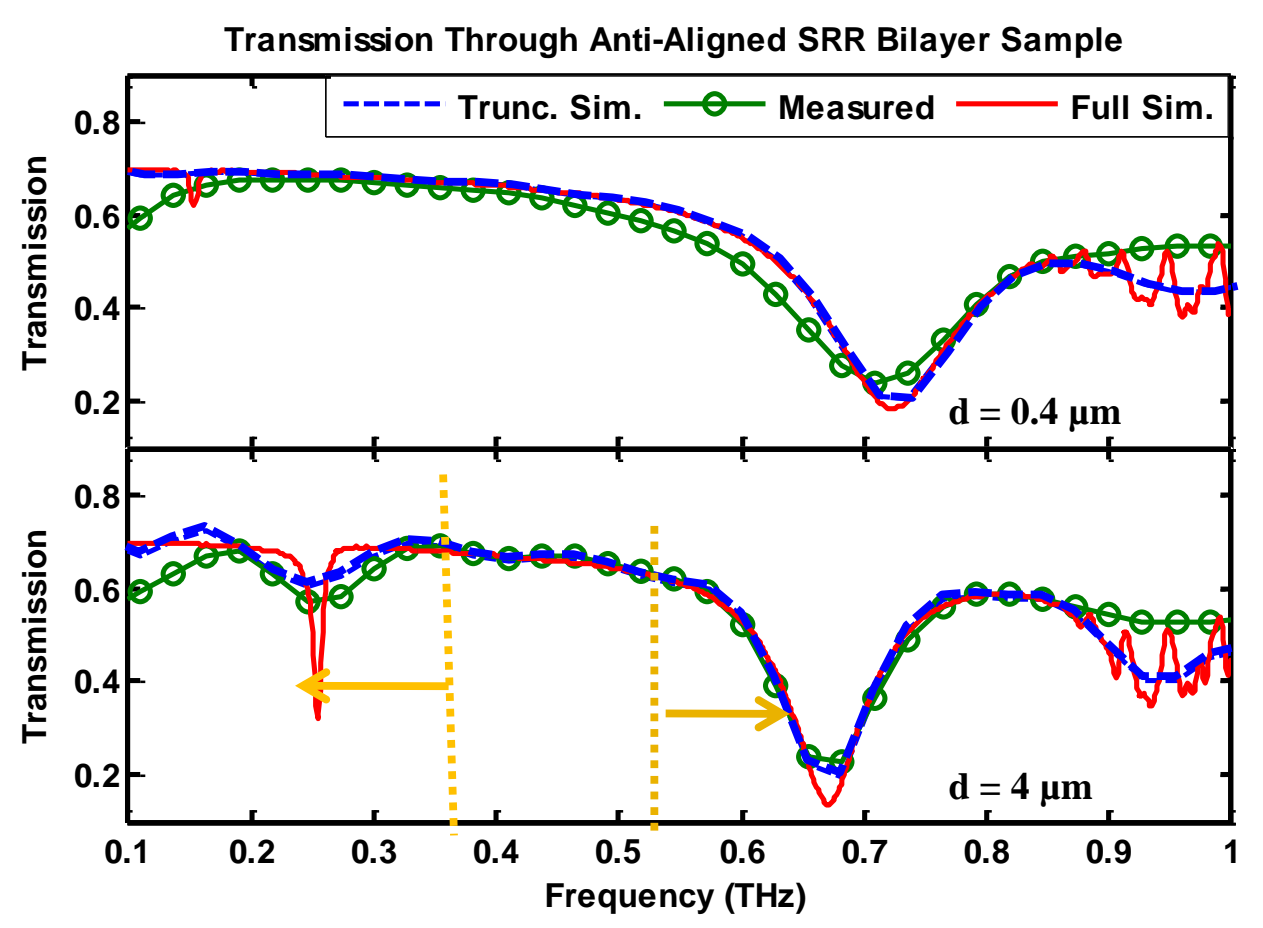

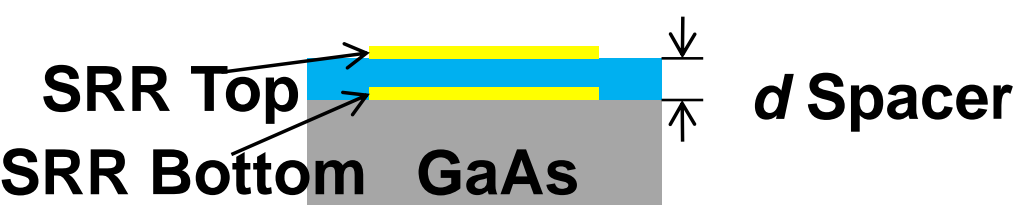

SRR Top Only $0.53 \mathrm{THz}$

SRR Bottom Only $0.34 \mathrm{THz}$ 


\section{Broadband Metamaterial}
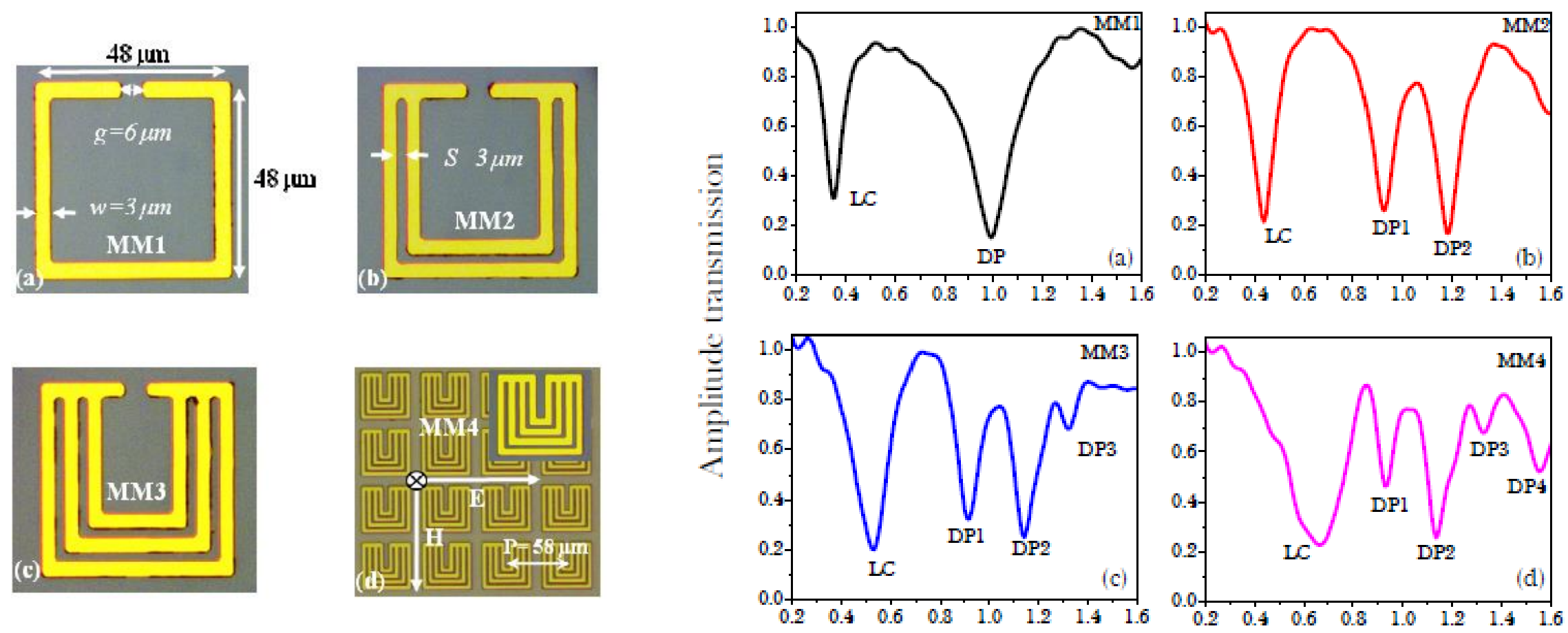

Frequency $(\mathrm{THz})$

D. R. Chowdhury et al., Optics Express 19, 15821 (2011) 


\section{Broadband Metamaterial}
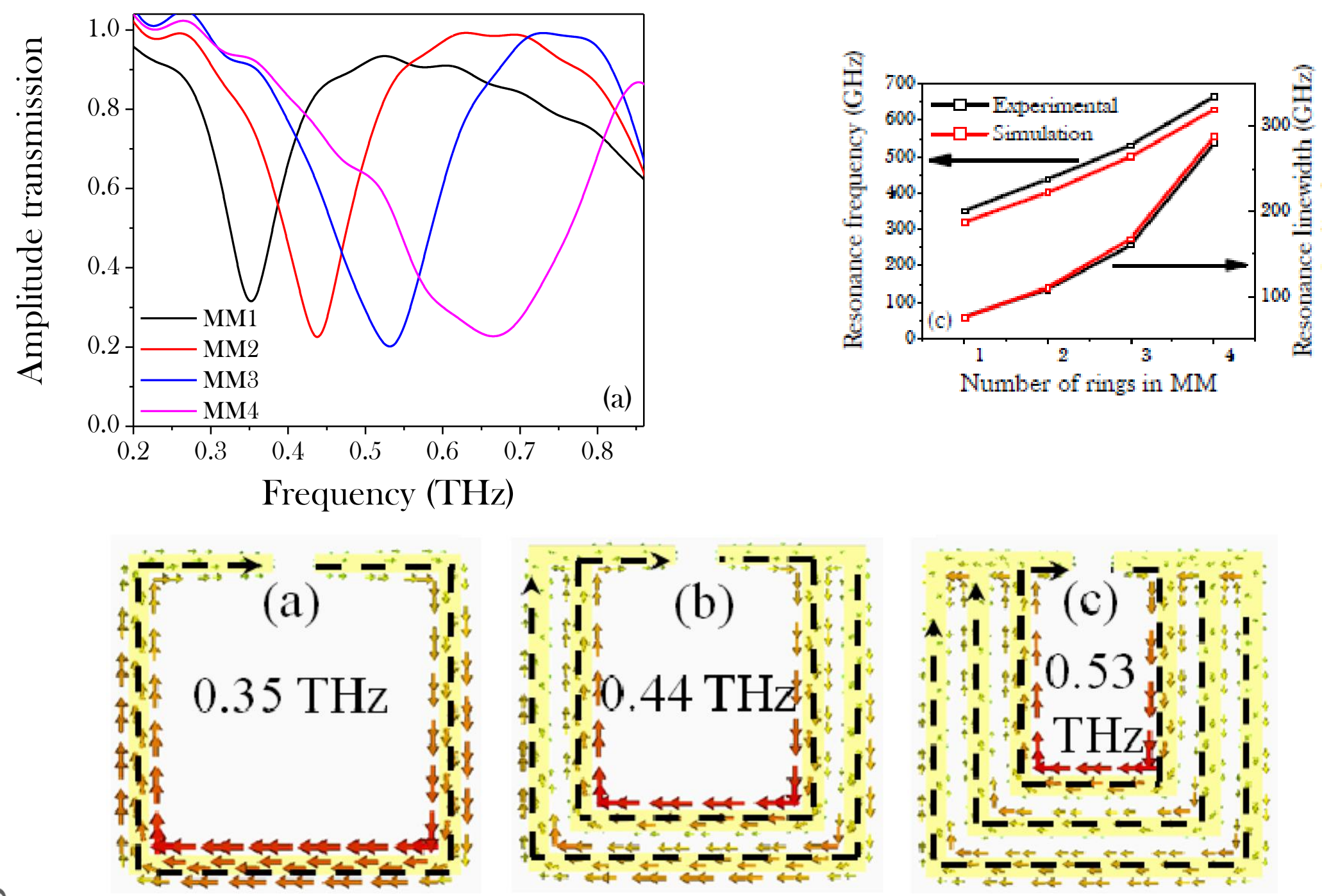